Florida International University

FIU Digital Commons

FIU Electronic Theses and Dissertations

University Graduate School

$11-15-2019$

\title{
Freeway Performance Measurement in a Connected Vehicle Environment Utilizing Traffic Disturbance Metrics
}

Leila Azizi

Florida International Univeristy, laziz001@fiu.edu

Follow this and additional works at: https://digitalcommons.fiu.edu/etd

Part of the Civil Engineering Commons, and the Transportation Engineering Commons

\section{Recommended Citation}

Azizi, Leila, "Freeway Performance Measurement in a Connected Vehicle Environment Utilizing Traffic Disturbance Metrics" (2019). FIU Electronic Theses and Dissertations. 4319.

https://digitalcommons.fiu.edu/etd/4319

This work is brought to you for free and open access by the University Graduate School at FIU Digital Commons. It has been accepted for inclusion in FIU Electronic Theses and Dissertations by an authorized administrator of FIU Digital Commons. For more information, please contact dcc@fiu.edu. 


\section{FLORIDA INTERNATIONAL UNIVERSITY}

Miami, Florida

\section{FREEWAY PERFORMANCE MEASUREMENT IN A CONNECTED VEHICLE ENVIRONMENT UTILIZING TRAFFIC DISTURBANCE METRICS}

A dissertation submitted in partial fulfillment of the requirements for the degree of DOCTOR OF PHILOSOPHY

in

CIVIL ENGINEERING

by

Leila Azizi

2019 
To: Dean John L. Volakis

College of Engineering and Computing

This dissertation, written by Leila Azizi, and entitled Freeway Performance Measurement in a Connected Vehicle Environment Utilizing Traffic Disturbance Metrics, having been approved in respect to style and intellectual content, is referred to you for judgment.

We have read this dissertation and recommend that it be approved.

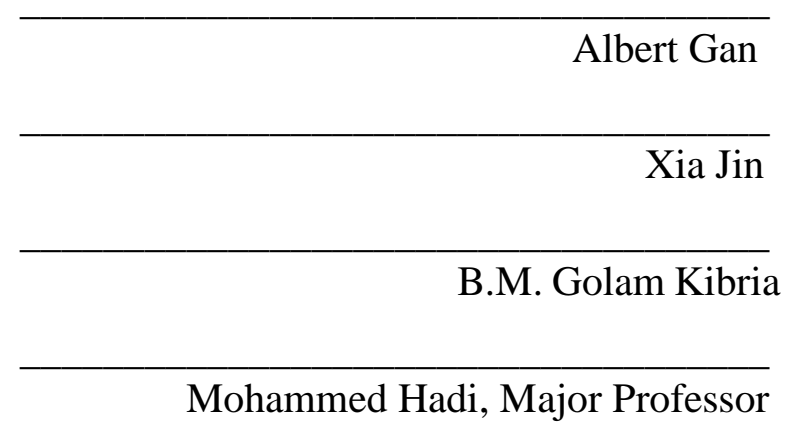

Date of Defense: November 15, 2019

The dissertation of Leila Azizi is approved.

Dean John L. Volakis
College of Engineering and Computing

Andrés G. Gil

Vice President for Research and Economics Development and Dean of the University Graduate School

Florida International University, 2019 
(C) Copyright 2019 by Leila Azizi

All rights reserved. 


\section{DEDICATION}

To My Lovely Parents 


\section{ACKNOWLEDGMENTS}

I would like to express my appreciation to my amazing advisor, Dr. Mohammed Hadi, for his continuous guidance and countless suggestions during all these years. Thanks to Dr. Hadi for always being constructive and providing great advice at every stage of my Ph.D. journey.

I am also grateful to Dr. Yan Xiao and Dr. Tao Wang for their endless support.

I also would like to thank all other members of my Ph.D. committee, Dr. Albert Gan, Dr. Xia Jin and Dr. B.M. Golam Kibria for their invaluable time reviewing my dissertation and providing valuable feedback.

Furthermore, I would like to acknowledge the US Department of Transportation (USDOT) through Southeastern Transportation Research, Innovation, Development and Education Center (STRIDE), for providing financial support.

Last but not least, special thanks to all my family members for their love and support. 


\section{ABSTRACT OF THE DISSERTATION \\ FREEWAY PERFORMANCE MEASUREMENT IN A CONNECTED VEHICLE \\ ENVIRONMENT UTILIZING TRAFFIC DISTURBANCE METRICS}

by

\section{Leila Azizi}

Florida International University, 2019

Miami, Florida

\section{Professor Mohammed Hadi, Major Professor}

The introduction of connected vehicles, connected and automated vehicles, and advanced infrastructure sensors will allow the collection of microscopic measures that can be used in combination with macroscopic measures for better estimation of traffic safety and mobility. This dissertation examines the use of microscopic measures in combination with the usually used macroscopic measures for traffic congestion evaluation, traffic state categorization, traffic flow breakdown prediction, and estimation of traffic safety. The considered macroscopic measures are the mean speed, traffic flow rate, and occupancy. The investigated microscopic measures for the stated purpose are: standard deviations of individual vehicle's speeds, standard deviation of vehicles' speed, and disturbance metrics. The utilized disturbance metrics to capture the stop-and-go operations are: the number of oscillations and a measure of disturbance durations in terms of the time exposed time-tocollision (TET), which has been used in other studies as a safety surrogate measure. However, this measure of disturbance duration requires the location and speed of both the leading and following vehicles and therefore cannot be measured accurately with low sample sizes of connected vehicles $(\mathrm{CV})$. Thus, this study derived a model to estimate this 
measure based on speed parameters. The developed model was tested using real-world trajectory data from two locations that were not used in the development of the model.

Moreover, the percentage of vehicles in the platoon and the platoon size distribution were evaluated as additional indicators of congestion. The relationship between the platooning and disturbance metrics and the speed parameters were further explored. It is recognized that the parameters required to identify the platoons, such as the time headway, will not be available based on data from low market penetrations of $\mathrm{CV}$. Thus, a model was developed that utilize other measures for the estimation of the platooning measures at lower CV market penetrations.

For the purpose of traffic state recognition and prediction, first, the study used a hybrid of two unsupervised clustering techniques to classify traffic states into "breakdown" and "non-breakdown". The study found that adding the disturbance metrics in data clustering when identifying the traffic states will result in better traffic state recognition and traffic flow breakdown identification by capturing the disturbances in the traffic stream. The categorized traffic state was then used as a binary response to the macroscopic and microscopic measures, as features, to train supervised machine learning techniques for predicting traffic flow breakdown in the following 5-minute interval in real-time operations. The study found that the utilizing disturbance and safety surrogate metrics in the real-time classification of traffic flow state increases the accuracy of prediction. Also, the study showed that the investigated disturbance metrics and associated models and thresholds are significantly related to crash frequencies and thus can be used in the activation of transportation management strategies to reduce the probability of unsafe traffic and ease traffic disturbances that have adverse impact on traffic safety. 


\section{TABLE OF CONTENTS}

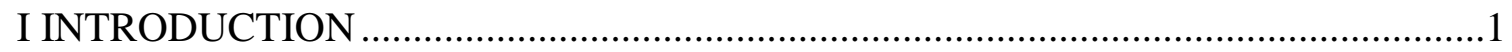

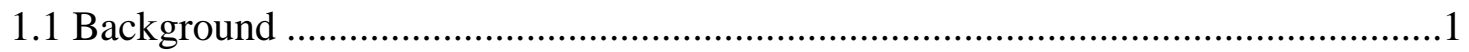

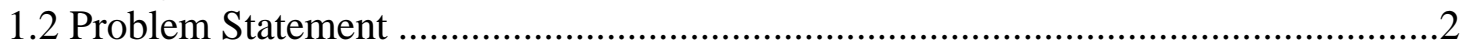

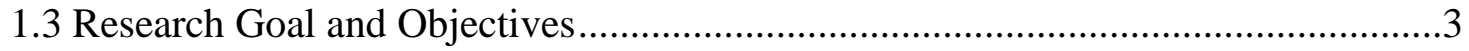

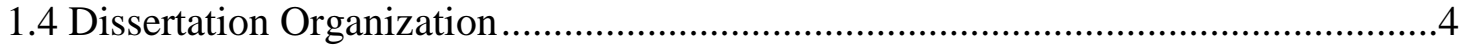

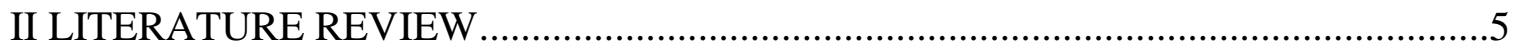

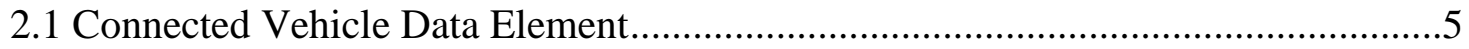

2.1.1 Data from Real-World Deployments of Connected Vehicles .......................... 6

2.1.2 Mapping Performance Measures to System Applications ............................... 7

2.2 Platooning Measures ........................................................................................

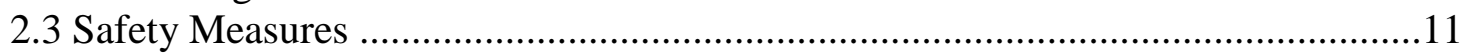

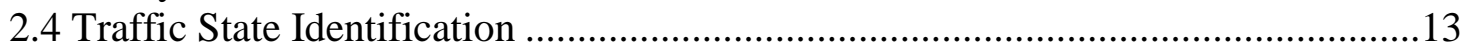

2.5 Traffic Flow Breakdown Prediction.............................................................16

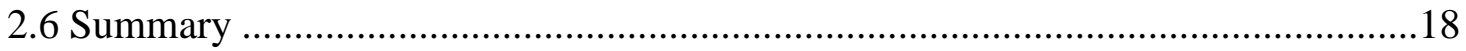

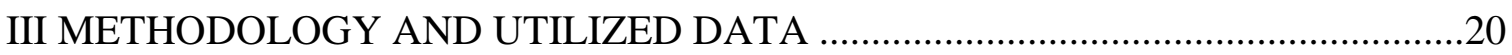

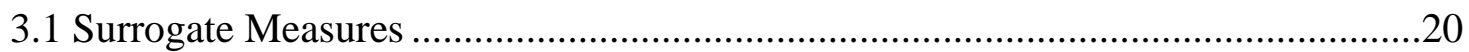

3.1.1 Standard Deviations of Speeds ................................................................ 20

3.1.2 The Index of Time Exposed Time-to-Collision (TETIndex) ……………..... 21

3.1.3 Number of Oscillations (NO) …………………..................................... 22

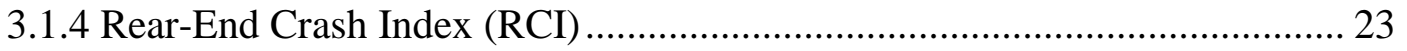

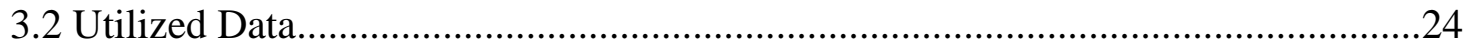

3.2.1 Microscopic Simulation ………………………..................................... 25

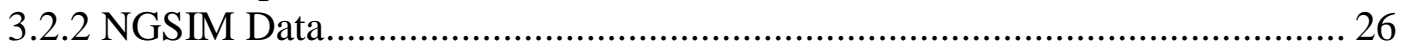

3.2.3 Connected Vehicle Data ............................................................................. 27

3.2.4 Descriptive Statistics of Utilized Data .......................................................... 28

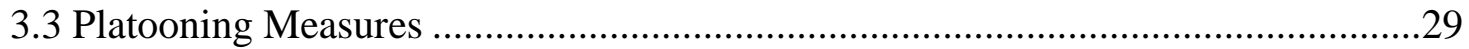

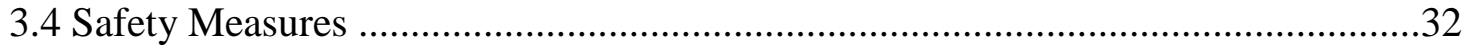

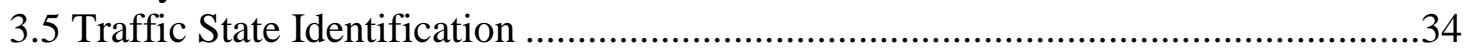

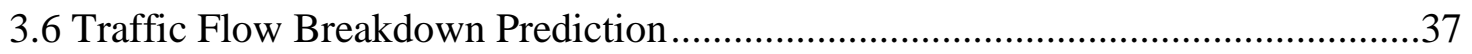

3.7 Summary …………................................................................................

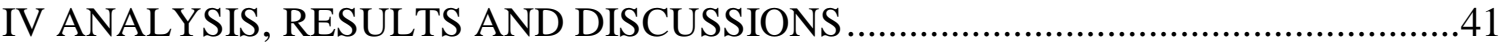

4.1 Relationships between Parameters ......................................................................41

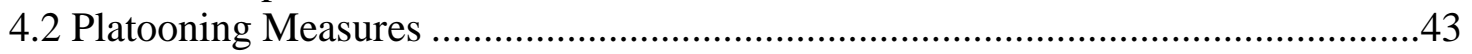

4.2.1 Platoon Percentage Determination .............................................................. 44

4.2.2 Determination of Platoon Size Distribution ................................................ 51

4.2.3 Relationship between Platooning and Disturbance Metrics .......................... 54 
4.3 Safety Measures

4.3.1 Developying Model to Estimate TETindex at Low Market Penetration of CV Data....

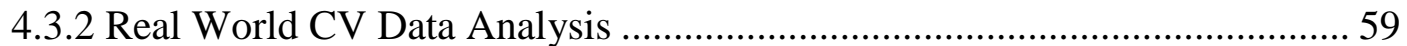

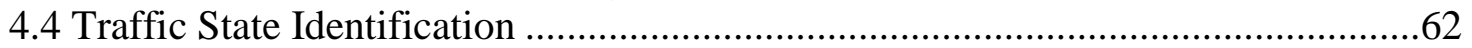

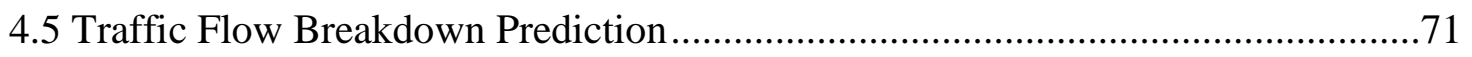

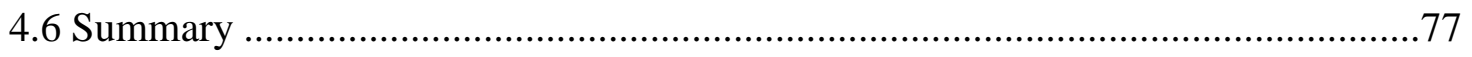

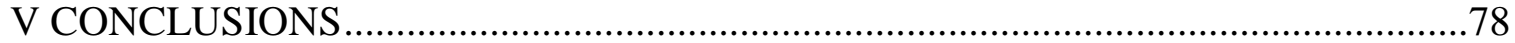

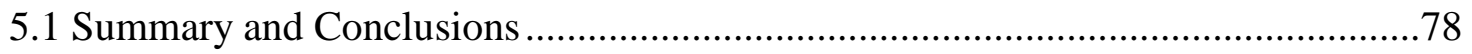

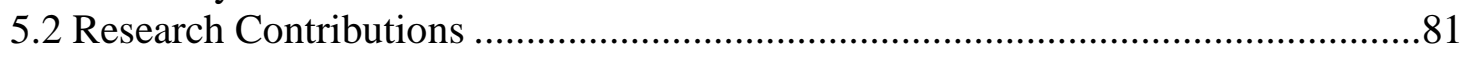

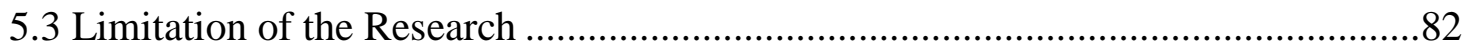

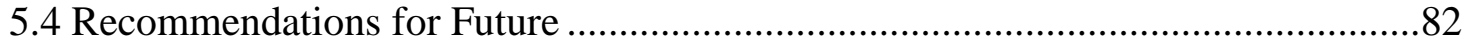

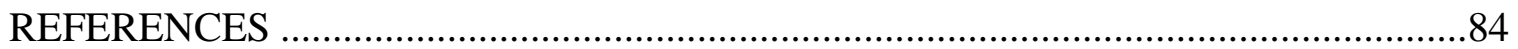

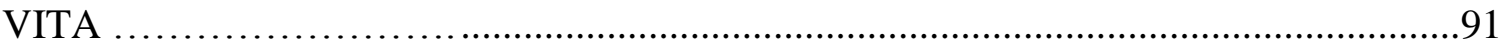




\section{LIST OF TABLES}

TABLE

PAGE

Table 2-1: Available CV Data on Freeways from US Funded Deployments......................6

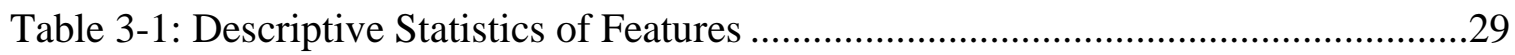

Table 4-1: Developed Equations to Estimate the Percent in Platoon ................................47

Table 4-2: The Quality of the Estimation of the Percentage of Vehicles in Platoon with Different Market Penetrations...........................................................................49

Table 4-3: Platoon Size Percentage based on All Trajectories ........................................52

Table 4-4: t-Test and $\chi 2$ Test for Different Market Penetration Levels ............................53

Table 4-5: Comparison of the Platoon Size Distribution Estimated with the 20\% and 100\% CV Market Penetration Levels ...........................................................54

Table 4-6: Coefficients and Quality of The TETIndex Estimation Based on PLS

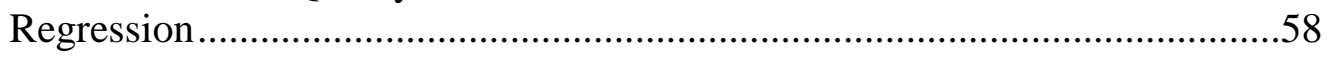

Table 4-7: The Quality of Estimations of the Developed Regression Model for Different Sample based on NGSIM Data

Table 4-8: Summary of Tuned Parameters and Model Accuracy with Different Input Variables

Table 4-9: Percentage of Traffic States in the Breakdown Cluster (Group1) and NonBreakdown Cluster (Group2) when Using Different Combinations of Features

Table 4-10: Evaluations of the Derived Clusters

Table 4-11: Comparison of the Selected Clustering with a Deterministic Value of Speed and with Clustering based on Speed-Occupancy in Capturing the Amount of Disturbances in the Uncertain Phase (50 fps $<$ Mean Speed $<70 \mathrm{fps}$ ).

Table 4-12: The Results of Selected Clustering in Capturing Disturbances in Cluster of Breakdown 
Table 4-13: Summary of Tuned Parameters with Three Scenarios for Each Classifier with

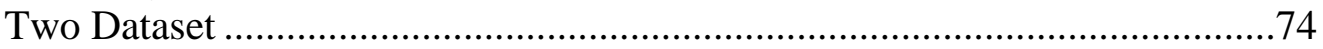

Table 4-14: Performance of the Three Classifiers in State Prediction based on Simulation

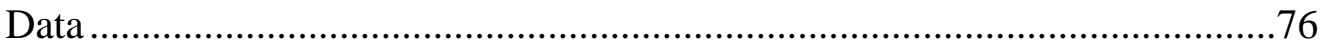

Table 4-15: Performance of the Three Classifiers in State Prediction based on Real-World

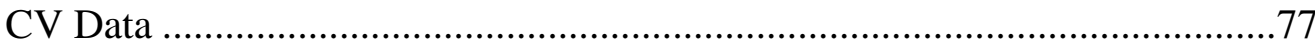




\section{LIST OF FIGURES}

FIGURE

PAGE

Figure 2-1: TTC Profile with TET and TIT (Minderhoud \&Bovy,2001)...................... 12

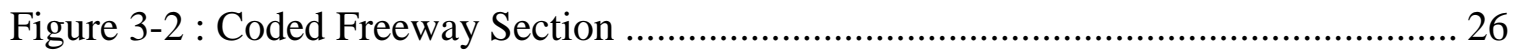

Figure 3-2: NGSIM Program Study Areas (a) I-80 and (b) US101 .............................. 27

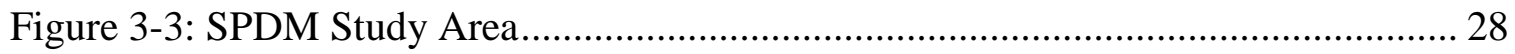

Figure 3-4: Methodology Framework of the Assessment of Safety ............................... 34

Figure 4-1: Fundamental Diagram of the Macroscopic Traffic Features ........................ 41

Figure 4-2: The Relationship between Space Mean Speed and (a) SDv and (b) SDt ...... 42

Figure 4-3: The Relationship between Disturbances with Mean Speed, SDv and SDt.... 44

Figure 4-4: The Relationship between SDt and The Percentage of Vehicles in the

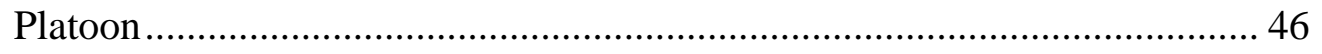

Figure 4-5: The Relationship between SDt and The Space Mean Speed ....................... 47

Figure 4-6: Platoon Percentages Estimated Using Different Headway Thresholds ......... 50

Figure 4-7: Relationship between Disturbance Metrics with NVP ............................ 55

Figure 4-8: The Relationship between RCI and (a) TETIndex and (b) NO ................... 56

Figure 4-9: Variable Importance as Identified by the Random Forest .......................... 60

Figure 4-10: Time-Space for the Two Examples of the Visual Inspection ..................... 64 
Figure 4-11: The Relationship between Disturbances with SDv

Figure 4-12: Data Clusters in the Test Segment of the NGSIM US101 Dataset............. 70

Figure 4-13: Variable Importance Ranking Using Random Forest Technique (a) The Simulation Dataset (b) The Real-World CV Dataset................................ 72 


\section{LIST OF ACRONYMS}

\begin{tabular}{|c|c|}
\hline AASHTO & American Association of State Highway and Transportation Officials \\
\hline ANN & Artificial Neural Networks \\
\hline BSM & Basic Safety Message \\
\hline $\mathrm{CV}$ & Connected Vehicles \\
\hline CAV & Connected and Automated Vehicles \\
\hline DSRC & Dedicated Short-Range Communication \\
\hline FR & Flow Rate \\
\hline FHWA & Federal Highway Administration \\
\hline GMM & Gaussian Mixture Models \\
\hline $\mathrm{HCM}$ & Highway Capacity Manual \\
\hline ITS & Intelligent Transportation System \\
\hline JPO & Joint Program Office \\
\hline MAPE & Mean Absolute Percentage Error \\
\hline NGSIM & Next Generation SIMulation \\
\hline NVP & Number of Vehicles in the Platoon. \\
\hline NCHRP & National Cooperative Highway Research Program \\
\hline NO & Number of Oscillations \\
\hline $\mathrm{OBU}$ & On Board Unit \\
\hline OOB & Out Of Bag \\
\hline PET & Post Encroachment Time \\
\hline PLS & Partial Least Square \\
\hline
\end{tabular}




\begin{tabular}{ll} 
PCA & Principal Component Analysis \\
RMSE & Root Mean Squared Error \\
RF & Random Forest \\
RSU & Roadside Units \\
RCI & Rear-End Crash Index \\
RBF & Radial Basis Function \\
SVM & Support Vector Machine \\
SDv & Average of Standard Deviations of Individual Vehicle's Speeds \\
SDt & Standard Deviation of Vehicles' Speed \\
SQL & Procedural Language extension to Structured Query Language \\
SAE & Society of Automotive Engineers \\
SPMD & Safety Pilot Model Deployment \\
SC & Silhouette Coefficient \\
TSMO & Transportation System Management and Operations \\
TMC & Transportation Management Center \\
TTC & Time-to-Collision \\
TET & Time Exposed Time-To-Collision \\
TIT & Time Integrated Time-To-Collision \\
VISSIM & Verkehr In Stadten Simulation \\
V2V & Vehicle-to-Vehicle communication \\
V2I & Vehicle-to-Infrastructure communication \\
X2X & Vehicle-to-everything \\
\hline
\end{tabular}




\section{CHAPTER I INTRODUCTION}

\subsection{Background}

Transportation system performance is a key component in congestion management, traffic safety management, setting agency priorities, and making policy decisions. Emerging connected and automated vehicle (CAV) technologies, shared autonomy, and shared mobility will significantly affect the demand and supply of the transportation network. They will also increase data quantity and quality, allowing the use of better performance measures and better estimation of existing measures.

The increase in the market penetration of connected vehicles $(\mathrm{CV})$ in the coming years will provide an important source of data for planning, planning for operations, and operations and management of transportation systems. The improved quality, quantity, details, and types of data provided by CV will allow for a better estimation of system performance, and the development and application of more effective strategies based on this estimation. Transportation System Management and Operations (TSMO) agencies are currently collecting data using point detectors, automatic vehicle identification technologies such as Bluetooth and $\mathrm{Wi}-\mathrm{Fi}$ readers, video analytics, and private sector vendor data. The parameters currently obtained and used based on these data sources usually include volume, speed, occupancy/density, and travel time measurements. The National CV Field Infrastructure Footprint Analysis document, produced by the American Association of State Highway and Transportation Officials (AASHTO) (Wright et al., 2014) recommends that public agencies should assess and trade-off the opportunities to use connected vehicle probe data aggregation and processing versus the continued deployment, 
operations, and maintenance of traditional ITS vehicle detection versus purchasing private sector data.

\subsection{Problem Statement}

Due to the limited data availability, the traffic mobility and safety performance estimations in real-time operations have been mainly based on the three fundamental macroscopic measures (speed, occupancy, and volume). The introduction of connected vehicles, connected automated vehicles, and advanced infrastructure sensors will allow the collection of microscopic measures that can be used in combination with the macroscopic measures for better estimation of traffic mobility and safety than what can be done with the macroscopic measures by themselves.

There are a number of studies that have investigated using CV data for performance measurement. However, these studies have mainly focused on measures that can be estimated by existing technology. Also, there have been limited efforts to investigate the potential of taking advantage of the more detailed data obtained from $\mathrm{CV}$ in deriving additional microscopic measures for use in assessing system operations. The availability of $\mathrm{CV}$ data will allow for the collection of parameters such as the distributions of the time headways between vehicles, variations of the speed between vehicles, variations of the speed of each vehicle, and variations of acceleration of each vehicle. A relatively low market penetration of $\mathrm{CV}$ may be required for estimating some of the detailed measures, while other measures will require high market penetrations to produce accurate results. Measures such as time headway, volume, and density will not be available based on data from low market penetrations of $\mathrm{CV}$. Thus, other surrogate measures are needed for the estimation of performance measures at lower CV market penetrations. 
Based on NCHRP report 551(NCHRP, 2006), the most commonly used criteria by agencies to select performance measurements for utilization are: easy to understand, well defined and quantifiable, describing existing conditions, predictability, accuracy of precision, variability by transportation alternatives, and consistently interpretability. The newly derived measures will provide agencies with additional capabilities to estimate system performance.

\subsection{Research Goal and Objectives}

The goal of this study is to investigate the use of microscopic measures that can be estimated in real-time operations based on $\mathrm{CV}$ data in combination with the commonly used macroscopic measures to estimate and predict system performance. For this purpose, this study defines additional microscopic measures to quantify disturbances by individual's vehicles. The disturbance metrics are defined to capture the stop-and-go operations and include the number of oscillations and a measure of disturbance durations in terms of the time exposed time-to-collisions, which has been used in other studies as a safety surrogate measure. An oscillation is defined as a deceleration phase followed by an acceleration phase. The time exposed time-to-collisions was derived from time-to-collision in the evaluation of the risk of collision. These existing measures are reformed and used as disturbance metrics in congestion evaluation, traffic state recognition, traffic breakdown prediction, and safety assessment using trajectory data and low market penetrations of CV data. The findings from this research will improve agency decision-making and allow optimized operations and better outcome performance. 
The specific objectives of this study are:

- Developing methods for traffic state identification utilizing defined disturbance metrics

- Developing methods for real-time prediction of traffic state utilizing defined disturbance metrics

- Developing methods for real-time traffic safety estimation utilizing defined disturbance metrics

- Assessing the developed models at low market penetrations of CV data

\subsection{Dissertation Organization}

This dissertation includes five chapters. Chapter II presents a review of CV data and literature review of existing performance measurement for congestion evaluation, traffic state recognition and prediction, and traffic safety. Chapter III discusses the assessment methodologies, machine learning methods, and utilized data to attain the stated objectives. Chapter VI presents the results, analyses and discussions. Chapter V summarizes the finding of this study and provides recommendations for future study. 


\section{CHAPTER II}

\section{LITERATURE REVIEW}

This chapter first provides information about CV data elements, available real word CV data, and its applications to transportation system. It then presents a literature review related to study's purposes including: (a) platooning formation (b) traffic state classification, (c) traffic flow state prediction and (d) safety assessment.

\subsection{Connected Vehicle Data Element}

Connected vehicle data are generated from vehicles and communicated to either the roadside units (RSU) or central facilities for processing and use. These data are useful for mobility, safety, and environmental applications. Obtaining some of the useful data elements requires connection to the vehicles on-board diagnostic port (OBD-II). The data is transmitted using connected vehicle messages utilizing dedicated short-range communication (DSRC) or other communication technologies such as cellular communications. The connected vehicle message types and components are specified in the Society of Automotive Engineers (SAE) J2735 standards (SAE International, 2009).

The basic safety message (BSM), specified in J2735, contains vehicle safety-related information broadcasted to surrounding vehicles, but can be also sent and/or captured by the infrastructure. The BSM, as defined in the J2735 standards, consists of two parts. Part 1 is sent in every BSM message broadcasted ten times per second. It contains core data elements, including vehicle position, heading, speed, acceleration, steering wheel angle, and vehicle size. Part 2 consists of a large set of optional elements such as precipitation, air temperature, wiper status, light status, road coefficient of friction, antilock brake system 
activation, traction control system activation, and vehicle type. However, not all of these data elements are currently available from every vehicle.

$\mathrm{CV}$ data communication involves communications between vehicles, infrastructure and road users to talk with each other. This is normally categorized as Vehicle to Vehicle (V2V), Vehicle to Infrastructure (V2I), and Vehicle to Everything (V2X) such as to pedestrians and bicycles. V2I communication provides significant opportunity to collect $\mathrm{CV}$ data for use in measuring system performance.

A key challenge is the collection, storage and analysis of data from connected vehicles. The amount of this data is considerably larger than traditional transportation data collected by existing sensors such as speed, volume, and occupancy measurements. CV offers the opportunity to collect detailed microscopic data from each vehicle ten times per second.

\subsubsection{Data from Real-World Deployments of Connected Vehicles}

One of the important consideration of the real-world deployments of the US Department of Transportation's intelligent transportation systems, Joint Program Office (JPO) is to share the collected ITS including CV data with the users. To date, the available connected vehicles data on freeways is summarized in Table 2-1 (ITS DataHub).

\section{Table 2-1: Available CV Data on Freeways from US Funded Deployments}

\begin{tabular}{|l|l|l|}
\hline CV Data & Short Description & Location \\
\hline $\begin{array}{l}\text { Safety Pilot Model } \\
\text { Deployment (SPMD) }\end{array}$ & $\begin{array}{l}\text { This data provides BSM, vehicle trajectories, } \\
\text { and various driver-vehicle interaction data }\end{array}$ & $\begin{array}{l}\text { Ann Arbor, } \\
\text { Michigan }\end{array}$ \\
\hline $\begin{array}{l}\text { Wyoming CV Pilot } \\
\text { BSM Sample }\end{array}$ & This is a live running log of sanitized BSM & Wyoming \\
\hline $\begin{array}{l}\text { Tampa CV Pilot } \\
\text { BSM Sample }\end{array}$ & $\begin{array}{l}\text { Generates data from the interaction between } \\
\text { vehicles and between vehicles and } \\
\text { infrastructure }\end{array}$ & Tampa, FL \\
\hline
\end{tabular}




\subsubsection{Mapping Performance Measures to System Applications}

The Wyoming CV pilot demonstration team has identified the applications of performance measures in nine categories focusing on improvement of traffic safety and mobility (Hartman et al., 2016). The measure applications were categorized in: (1) improving performance during bad weather condition, (2) improving ability of traffic management centers (TMC) to generate alerts and advisories, (3) efficiently disseminate traveler information, (4) effectively disseminating and receiving I2V and V2I alerts from the TMC, (5) improving information to fleet manager, (6) effectively transmitting and receiving $\mathrm{V} 2 \mathrm{~V}$ messages, (7) automatic emergency notification of crashes, (8) reducing speed variation, and (9) reducing crashes.

\subsection{Platooning Measures}

This section first defines platoon and then reviews existing studies that developed and utilized methods to estimate platooning in congestion evaluation. These study mainly focus on the estimation of the percentage of vehicles in the platoon and the platoon size distribution based on macroscopic and microscopic traffic flow parameters.

As the flow rate increases, two or more vehicles will form platoons. Platooning is important as it has serious implications on traffic operations. The Highway Capacity Manual (HCM) ( 2016) defines platoon as "a group of vehicles traveling together either voluntarily or involuntarily because of signal control, geometric or other factors." A vehicle with a higher desired speed catches up with a slow vehicle and is forced to follow it, reducing its speed to maintain its desired following distance.

Traffic flow breakdown on a freeway and its stochastic nature are strongly related to the platooning and platoon size (Shiomi et al., 2011). Shiomi et al. (2011) defined the 
traffic breakdown probability as a function of platoon size and its lead vehicle's speed, given the traffic flow rate. They also utilized a probability density function of the appearance of a platoon of size of "x," in which " $x$ " is the number of vehicles in the platoon. Thus, the likelihood of a platoons of a given size can be estimated utilizing this function. In addition to their use in determining the probability of breakdown, the platooning characteristics can be used as criteria to determine the level of service of a facility. When drivers are forced to adjust their speed and follow the leading vehicle, they perceive lower serviceability. Platooning also indicates lower maneuverability, in terms of the ability to change lanes, once again indicating a lower level of service. Furthermore, there is an increase in conflicts with the presence of platoons, indicating an impact on safety. A previous study showed that the number of platooned vehicles over one mile can be used as a better serviceability criterion in multi-lane highways, compared to density (Chatterjee et al., 2017). Thus, measuring or estimating platooning attributes, as part of traffic operations and management, will allow TSMO agencies to make better decisions to implement active traffic management strategies to reduce the probability of breakdown and improve the level of service. The estimation of the platooning characteristics on a freeway segment is important for both planning and operation analysis and can be used to assess the level of operations and service of traffic, as well as to potentially predict the safety of the traffic stream.

The relationship between platooning and traffic flow stability has been a subject of research in traffic flow theory and applications. Platooning characteristics have been associated with three types of instability that represent increasing levels of instability: local instability, platoon (or string) instability, and traffic flow instability. Determining the 
distribution of platoon size and the distribution of the inter-platoon gaps can be used in assessing the level of instability. Traffic flow is considered unstable if it contains enough long platoons and short inter-platoon gaps resulting in the instability within one platoon transferring to the next platoon and the disturbance continuing to grow in amplitude (Pueboobpaphan \& Arem, 2011).

The review of literature shows that the time headway has been widely used for platoon identification. Different thresholds of time headway, ranging from 3.0 seconds to 6.0 seconds, have been used in the past to identify platooning vehicles. Lay (1986) suggested three groups for time headway. When the headway is less than 2.5 seconds, the traffic is recognized to be following (in the platoon). When it is between 2.5 seconds and 9.0 seconds, the traffic is considered to be either following or free (not platooning), and when it is more than 9.0 seconds, the traffic is considered free. Shimoi et al. (2011) defined platoons as vehicles whose speeds are strongly correlated with the speed of the lead vehicle. They studied the relationship between the time headway and the correlation coefficient in the speed of two successive vehicles and determined that the critical headway is 4.0 seconds. Rahman et al. (2012) used a time gap threshold of 4.0 seconds instead of time headway to define platooned vehicles. Vogel (2002) found that the 6.0-second time headway is optimal for identifying non-following vehicles. Al-Kaisy and Durbin (2009) identified vehicles as being in a platoon based on plotting the mean speed of the vehicles and their time headways. The time headway that indicates platooning was identified to be 3.0 seconds. The HCM points to 3.0 seconds as the threshold, although it recommends using the percentage of time spent following to determine the platooned vehicles. Gattis et al. (1997) used 5.0 seconds as a time headway criterion to define platoons. 
Yang et al.(2015) conducted empirical analysis on platoons from free flow to congestion flow at on-ramp bottlenecks in I-405 Santa Monica, California and I-95 Backlick, North Virginia. They found that the platoon time headway fits a normal distribution, with a mean of 1.42 seconds and standard deviation of 0.42 seconds in Backlick. In Santa Monica, it fits the lognormal distribution with a mean of 1.66 seconds and standard deviation of 0.84 seconds. They also found that the non-platoon vehicle time headway fits shifted negative exponential distribution with a mean of 3.01 seconds and standard deviation of 1.12 seconds.

Researchers also linked the position of a vehicle in a platoon with the standard deviation of speed. Jiang et al. (2015) conducted an experimental study of car-following behavior in a 25-car platoon using GPS data and found that the standard deviation of speed increases in a concave manner with the position of the vehicle in the platoon. Tian et al. (2015) investigated the growth pattern of traffic oscillation using US-101 trajectories data collected by the Next Generation SIMulation (NGSIM) program and produced the following relationship between the location of the vehicle in a platoon and its standard deviation of speed:

$$
\mathrm{SDV}=-10.4 \mathrm{e}^{\left(-\frac{\mathrm{NVP}}{94.29}\right)}+10.56
$$

where SDv is the standard deviation of speed of each vehicle $(\mathrm{m} / \mathrm{s})$ and NVP is number of vehicles in the platoon.

Researchers also investigated the variation in the percentage of vehicles in a platoon with traffic volume. Sun et al.(2005) examined the relationship between traffic volume and the percentage of vehicles platooning on a highway, with two lanes in each direction and short-term and long-term work zones in Illinois. They showed that as the volume increases, 
the percentage of platooning vehicles increases. The following equation was developed based on the collected data:

$$
\mathrm{FR}=-1.377+0.327 \times \ln (\mathrm{PIP})
$$

where FR is the hourly flow rate (vphl) ranging between 400 and 1,400 vphl, and PIP is the percentage of vehicles in the platoon.

\subsection{Safety Measures}

Rear-end collisions are a main safety concern on freeways, mainly caused by slow or stopped traffic. Because collisions are rare events, crash data for at least three years is required to have a sufficient sample size to assess traffic safety (Rahman \& Abdel-Aty, 2018). The safety assessment to support TSMO, particularly in real-time operations, does not have access to sufficient crash data to assess safety. To assess safety for a shorter period of time or when such data is not available, traffic conflicts have been used as a technique to assess the safety at a location ( $\mathrm{Li}$ et al., 2017; Rahman \& Abdel-Aty, 2018), with the assumption that the conflict statistics is correlated with the risk of actual collisions (Dijkstra et al., 2010; Lu et al., 2011). A conflict is a scenario where two drivers will likely collide without evasive action. In order to evaluate rear-end crashes, surrogate measures of safety have been proposed to allow the development of relationships between the likelihood of crashes and traffic stream flow parameters (Kuang et al., 2015; Z. Li et al., 2014). These measures have been widely used as indicators to evaluate rear-end crash risk and to quantify the number of conflicts. Examples are the time-to-collision (TTC), time

exposed time-to-collision (TET), time integrated time-to-collision (TIT), post encroachment time (PET) and deceleration rate to avoid the crash (DRAC) (Abdel-Aty \& Pande, 2005; Guido et al., 2012; Li et al., 2017; Peng et al., 2017; Rahman \& Abdel-Aty, 
2018). TTC is defined as the expected time for two vehicles, following each other, to collide if they remain at their present speed and on the same path (Hayward, 1972). The TET reflects the total time spent under dangerous traffic conditions, characterized by TTC values below a threshold value of TTC. TIT is a measure that represents the TTC lower than the threshold. TET and TIT were derived from TTC (Minderhoud \& Bovy, 2001). Figure 2-1 describes these measures. PET (Gettman \&Head, 2003) represents the time difference between vehicle leaving the area of encroachment and a conflicting vehicle entering the same area. DRAC defined as "rate at which crossing vehicles must deaccelerate to avoid collision with the conflicting vehicle" (Gettman \&Head, 2003). Moreover, since a rear-end crash may occur due to insufficient safety distance between the leading and the following vehicle, Oh et al. (2006) proposed a rear-end crash index (RCI) based on the safe stopping distance in car following to indicate dangerous conditions.

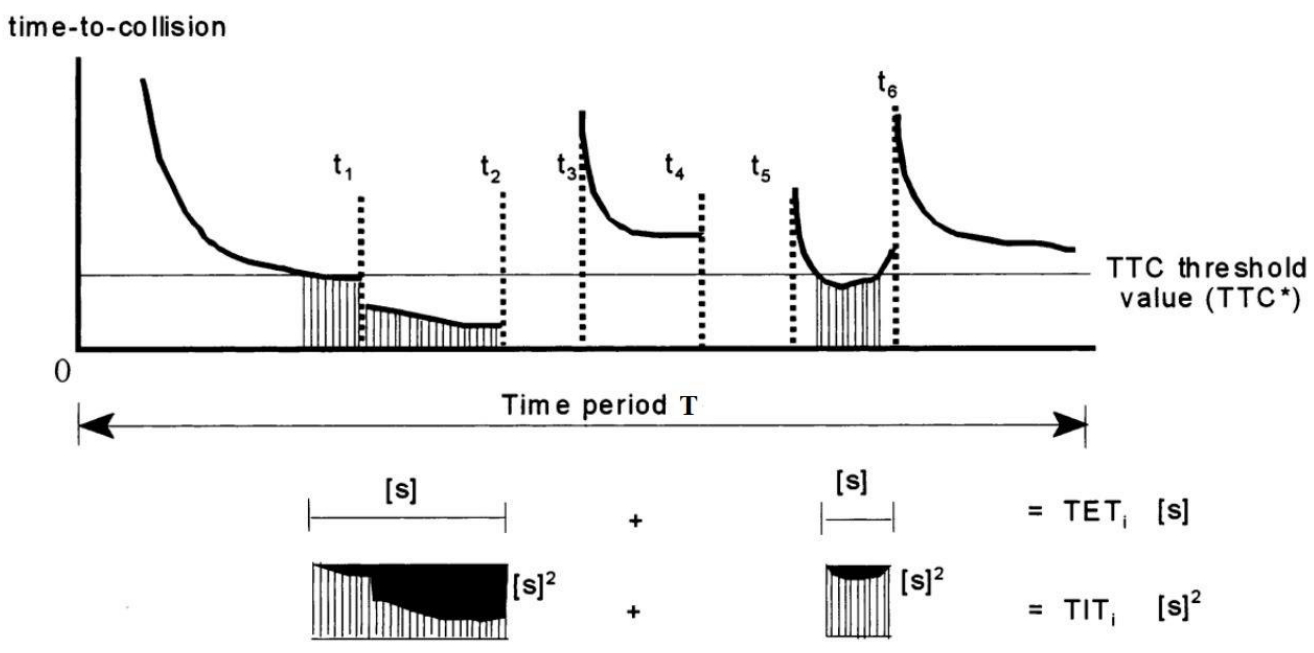

Figure 2-1: TTC Profile with TET and TIT (Minderhoud \&Bovy,2001)

The macroscopic traffic flow parameters (flow, speed, and density) have been used in safety performance estimation (Chang \& Xiang, 2003). However, these measures do 
not adequately capture the interactions among individual vehicles. A study by Zheng (2012) showed that crashes happen in congested conditions and in the transition conditions about six times and two times, compared to free flow conditions, respectively. There are limited studies that utilize microscopic traffic flow data in estimating the safety performance. Zheng ( 2012) reported that a combination of speed, speed variance and flow as a good indicator of traffic's chaos which has adverse impact on traffic safety. A study by Zheng et al. (2010) also showed that traffic oscillations are related to the standard deviation of speed and crash rate tends to increase as the standard deviation of speed increases. Other studies also found a direct relationship between the standard deviation of speed and safety (Abdel-Aty \& Pande, 2005; Kamrani et al., 2018; Lee et al., 2002). Arvin et al. (2019) used lateral and longitude accelerations of individual vehicles that were estimated based on CV data collected as part of the Safety Pilot Model Development (SPMD) as a measure of driver's volatility that impacts crash frequencies at intersections.

The above review of the literature indicates that although a number of studies have investigated using surrogate measures for safety performance assessment, there is limited work on utilizing microscopic parameters at the individual vehicle level, that will potentially become available from small market penetrations of emerging vehicle technology, to assess safety.

\subsection{Traffic State Identification}

The categorization and recognition of the traffic state, particularly the occurrence of breakdown, is critical to traffic flow analysis and effective traffic management and operations. Due to the data availability, the identification of the traffic states has been mainly based on the three macroscopic measures (speed, occupancy, and volume). The 
introduction of connected vehicles, connected automated vehicles, and advanced infrastructure sensors will allow the collection of microscopic measures that can be used in combination with the macroscopic measures for better recognition of the traffic state.

Understanding traffic flow breakdown mechanism and the probability of its occurrence is important in traffic analysis, management and operations. The causes of traffic breakdowns include: high traffic volume that exceeds the maximum allowable throughput and/or bottlenecks where the capacity drops due to disturbance caused by individual drivers such as lane changing and abrupt braking. The Highway Capacity Manual (HCM) procedure for basic freeway segments categorize the traffic states into six levels of service (LOS) and analysts have generally assumed that the breakdown occurs in the threshold between LOS E and F, where the demand exceeds capacity of the freeway segment. The traffic flow rate at breakdown can be lower than the estimated average roadway capacity, as defined in the HCM. Research efforts have confirmed that breakdown can happen stochastically and not at a deterministic value of capacity (Dong \& Mahmassani, 2012; Elefteriadou et al., 1995).

For modeling the probability of traffic breakdown, the classification of traffic conditions into congested and uncongested conditions is important. Such classification, if estimated and eventually predicted, will allow traffic management agencies to activate operational plans to address the adverse impacts of congestion. Traffic flow breakdown is usually defined as a speed drop of a certain amount when the traffic demand exceeds capacity. The HCM defines breakdown on freeways as a condition when the speed drops below a certain threshold (e.g., $40 \mathrm{mph}$ ) and/or by a certain amount (e.g., $10 \mathrm{mph}$ ) 
(Elefteriadou, 2017) and it is sustained at least for three time intervals (e.g., 15 minutes totally).

Researchers have used visual observations of the traffic flow-speed, flowoccupancy and speed-occupancy diagrams to evaluate breakdown using threshold values of speed, flow, density/occupancy, or combinations of these variables (Dehman, 2014; Kondyli et al., 2013; Laflamme \& Ossenbruggen, 2017; Yeon et al., 2009). The changepoint regression modeling was also used to identify the critical value to estimate the breakdown based on the speed-occupancy diagram (Kidando et al., 2019). Clustering models, which are unsupervised machine learning algorithms, such as the Gaussian mixture model (Kidando et al., 2018; Ko \& Guensler, 2005) and K-Means clustering (Elfar et al.,2018; Jingxin et al., 2013) have also been used to identify the traffic state based on speed and density. The speed threshold used to identify breakdown in previous studies widely varied from $25 \mathrm{mph}$ to $50 \mathrm{mph}$, showing that there is no agreed-on definition of breakdown (Kidando et al., 2019).

Some researchers also defined traffic regimes based on oscillations and categorized traffic conditions as non-oscillatory, damped oscillatory, and oscillatory regimes. The breakdown is associated with the oscillatory regime that indicate unstable conditions (Herman et al., 1959; Swaroop \& Rajagopal, 1999). A traffic condition is considered stable if the flow is able to handle disruptions without breaking down. Treiber and Kesting ( 2013) divided the traffic flow states into stable and unstable and recognized key factors of congested traffic instability such as disruption and its propagation probability. It was found that there is always a growth of perturbation in the congestion regime (Treiber \& Kesting, 2013). Treiber and Kesting (2013) defined five stability classes based on density, with two 
classes are unconditionally stable or unstable but the remaining three classes can be stable, unstable or metastable and there is a probability of traffic breakdown associated with these states. However, an experimental study by Jiang et al. (2018) showed that traffic instability is related more to speed rather than density. They also found that for the same average traffic speed, some experiments showed stable traffic flow while others showed unstable traffic flow. This indicates that speed by itself may not be a sufficient indicator of breakdown and other parameters are needed to indicate traffic instability. Some researchers assumed traffic flow to be stable if the slope of the flow-speed fundamental diagram is positive and is unstable if the slope is negative. However, it was shown that under the negative slope, the traffic flow is not necessarily unstable and depends on driving behaviors (Pueboobpaphan \& Arem, 2011). Chatterjee et al.(2017) also used platoon characteristics to determine three different traffic states: free flow, stable flow and constraint flow.

The majority of previous studies used either visual observation or clustering methods using macroscopic parameters (speed, flow, and density) to identify traffic state. However, the review of literature presented in this section indicates that other factors should be considered for accurate traffic state identification and prediction.

\subsection{Traffic Flow Breakdown Prediction}

The prediction of the traffic flow breakdown is critical to effective traffic management and operations. Active traffic management applications have generally used macroscopic traffic metrics in their decisions to activate operation plans. The traffic breakdown has been identified mainly based on thresholds of speed and/or occupancy/density. The critical speed or density at capacity, as identified in the highway capacity manual (HCM) can be used for this purpose. In terms of traffic flow, however, 
research efforts have confirmed that breakdown can happen stochastically and not at a deterministic value of capacity (Dong \& Mahmassani, 2012; Elefteriadou et al., 1995). As mentioned before, the introduction of connected vehicles, connected automated vehicles, and advanced infrastructure sensors will allow the estimation of microscopic traffic metrics that can be used in combination with the macroscopic measures commonly used for better identification of the traffic breakdown in terms of both mobility and safety.

Traffic flow breakdown is usually defined as a speed drop of a certain amount. There are numerous studies that investigated the traffic breakdown phenomena. The first probabilistic breakdown model was proposed in 1972 (Bullen, 1972) identifying the probability of breakdown as an increasing function of the flow rate. Wang et al. (2010) developed a model for predicting the breakdown probability based on the expected future density, utilizing Markov transition. Son et al. (2004) developed a probabilistic model of traffic breakdown, triggered by merging vehicles, by applying a wave propagation model with random disturbance. Elefteriadou et al. (1995) defined the breakdown mechanism as resulting from a large vehicle groups entering freeway through an on-ramp causing speed drops. Shiomi et al. (2011) defined the traffic breakdown probability as a function of platoon size and its lead vehicle's speed given the traffic flow rate. Ahn et al. ( 2017) studied a stochastic modeling of traffic breakdown for freeway merge bottlenecks with consideration of headway distribution. Chen et al. (2014) proposed a model for traffic breakdown, caused by perturbations of on-ramp merging vehicles, based on queuing theory. Dong et al. (2012) studied traffic breakdown, focusing on disturbance caused by speed changes of the lead vehicle and the propagation by followers. Kondyli (2009) also 
studied breakdown probability at freeway ramp merge based on the stochasticity of driver behaviors in accepting gaps and making decisions.

With the rapid advancement of machine learning, it is possible to utilize large amount of data including microscopic traffic data that includes detailed trajectory data to improve the accuracy of traffic state identification and prediction. The machine learning methods are mainly categorized in three categories: naïve, parametric, and non-parametric methods (Van \&Van, 2012). There are numerous studies in transportation engineering on using various machine learning methods in a wide variety of applications. However, there is a limited research on using machine learning methods in conjunction with highresolution data in real-time traffic state estimation. Elfar et al. ( 2018) used trajectory data to predict congestion utilizing speed and standard deviation of speed. There are also existing studies that used deep learning methods in traffic congestion and trajectory prediction (Khajeh Hosseini \& Talebpour, 2019; Zhang et al., 2019). However, these existing studies (Elfar et al., 2018; Khajeh Hosseini \& Talebpour, 2019) focused on the classification of congestion for short periods of time in the future (10 seconds/ 20 seconds) and did not combine mobility and safety metrics in the prediction. In addition, these studies used data that were collected only at congested conditions and do not covered all traffic conditions (Elfar et al., 2018; Zhang et al., 2019).

\subsection{Summary}

This chapter presented a comprehensive literature review on platooning measures, safety measures, traffic state identification, and traffic flow breakdown prediction. It can be concluded that there have been limited efforts to investigate the potential of the more detailed data obtained from $\mathrm{CV}$ in deriving additional measures for use in assessing system 
operations. The review of the literature indicates that although a number of studies have investigated using surrogate measures for safety and traffic state performance assessment, there is limited work on utilizing microscopic parameters at the individual vehicle level, that will potentially become available from emerging vehicle technology, to assess safety and mobility. Also there is no study on the identification of microscopic measures' thresholds to justify activating plans to mitigate unsafe and congested conditions in realtime operations.

Some data required for better measurements of performance such as the time headway used in estimating the platooning characteristics and the TET, which is a surrogate measure to safety will not be available based on data from low market penetrations of $\mathrm{CV}$. For example, if the market penetration of $\mathrm{CV}$ is $5 \%$, then the percentage of vehicles following each other is $0.25 \%$. This means that there will be an opportunity for 25 headway measurements for each 10,000 passing vehicles. Therefore, there is a need for methods for the estimation based on surrogate measures that can be assessed using $\mathrm{CV}$ data at a relatively low market penetration of $\mathrm{CV}$. 


\section{CHAPTER III \\ METHODOLOGY AND UTILIZED DATA}

This chapter presents the surrogate measures, utilized data, and methods that were used to achieve the research objectives stated in Chapter I.

\subsection{Surrogate Measures}

Before providing more details on the utilized methodology and data, this section describes the microscopic traffic parameters investigated for use in the listed study' purposes and how they are calculated. A Procedural Language extension to Structured Query Language (PL/SQL) program was developed with Oracle SQL Developer to calculate these measures utilizing the trajectory datasets used in this study.

\subsubsection{Standard Deviations of Speeds}

The standard deviation of speed is related to the shockwave and platoon formation, which preludes traffic breakdown (Elfar et al., 2018). Some studies also showed that the increase in standard deviation of speed will result in traffic breakdown (Krauss et al., 1997; Kühne, 1984).The standard deviation of individual vehicle's speeds (SDv) and standard deviation of vehicles' speed (SDt) are calculated as follows:

$$
\begin{gathered}
\mathrm{SDt}=\sqrt{\frac{1}{\mathrm{n}-1} \sum_{\mathrm{i}=1}^{\mathrm{n}}\left(\mathrm{V}_{\mathrm{i}}-\overline{\mathrm{V}}\right)^{2}} \\
\mathrm{SDv}=\sqrt{\frac{1}{\mathrm{n}_{1}-1} \sum_{\mathrm{j}=1}^{\mathrm{n}_{1}}\left(\mathrm{~S}_{\mathrm{j}}-\overline{\mathrm{S}}\right)^{2}}
\end{gathered}
$$

where, $\mathrm{n}$ is the number of vehicles, $\mathrm{V}_{i}$ is the speed of vehicle $\mathrm{i}, \overline{\mathrm{V}}$ is the mean speed of vehicles, $\mathrm{n}_{1}$ is the number of speed data collected for a specific vehicle at 1/10th of a second time interval, $S_{j}$ is the speed of the vehicle at interval $j$, and $\bar{S}$ is the mean of individual vehicle's speeds. 


\subsubsection{The Index of Time Exposed Time to Collision (TETIndex)}

Time-to-Collision (TTC) is a primary conflict measure, introduced by Hayward (Hayward, 1972), and has been widely used as a surrogate safety measure for rear-end crashes. TTC is defined as the expected time for two vehicles, following each other, to collide if they remain at their present speed and on the same path (Hayward, 1972). It has been reported that lower TTC is a good indicator of the probability of collision, but cannot be directly linked to the severity of the collision. Balas et al. (Balas \& Balas, 2007) used the inverse of the TTC as an indicator of safety. Another type of TTC referred to as TTC $_{\text {Break }}$ (Peng et al., 2017) was proposed to consider situations, in which the leading vehicle stops suddenly. This parameter is calculated as follows:

$$
\operatorname{TTC}_{\text {Break }}(t)=\frac{x_{i-1}(t)-x_{i}(t)-\bar{L}}{v_{i}(t)} \text { if } v_{i}(t)>v_{i-1}(t)
$$

where $\operatorname{TTC}_{\text {Break }}(t)$ is the time to collision value of vehicle $i$ at time $t, x_{i}(t)$ is the position of vehicle $i$ at time $t, x_{i-1}(t)$ is the position of vehicle $i-1$ at time $t, v_{i}(t)$ is the speed of vehicle $i$ at time $t$, and $\bar{L}$ is the average length of vehicles.

An aggregate indicator, the TET, was introduced (Minderhoud \& Bovy, 2001) to assess the safety performance of monitored segment in space and time. The TET reflects the total time spent under dangerous traffic conditions, characterized by TTC values below a threshold value TTC*. The TET is calculated as follows.

$$
\begin{aligned}
& \operatorname{TET}(\mathrm{t})=\sum_{\mathrm{i}=1}^{\mathrm{n}} \delta_{\mathrm{t}} \Delta \mathrm{t} \\
& \delta_{\mathrm{t}}=\left\{\begin{array}{l}
1,0<\mathrm{TTC}_{\text {break }}(\mathrm{t}) \leq \mathrm{TTC}^{*} \\
0, \\
\text { else }
\end{array}\right. \\
& \mathrm{TET}=\sum_{\mathrm{t}=1}^{\mathrm{T}} \operatorname{TET}(\mathrm{t})
\end{aligned}
$$


where, $\Delta t$ is the time step of the trajectory data collection, which is usually set at 0.1 second in simulation, TTC* is the threshold, $\mathrm{n}$ is the number of vehicles, and $\mathrm{T}$ is the time period.

The TTC* threshold, referenced above is used to differentiate the unsafe car following conditions from the ones that are considered safe. According to the past research (Abdel-Aty \& Pande, 2005), the threshold values are usually set between 0.5 second to 3.5 second. Fan et al. (Fan et al., 2013) recommended using a TTC* threshold of 2 seconds in freeway merge areas to identify the conflicts. The sensitivity analysis in previous research also showed that changing the TTC* value within the investigated range does not have a significant effect on the results of the safety assessment ( Li et al., 2017). Based on the above review, this study uses TET with a threshold (TTC*) of 2 seconds, as the surrogate safety measure to be estimated based on CV trajectory data.

In this study, TET is calculated for each subsegment and normalized using Equation 3-5 to determine a TET index, which is proposed in this study as an index of disturbance duration.

$$
\text { TETIndex }=((\text { TET } / \mathrm{n}) / \mathrm{T}) \quad 0<=\text { TETIndex }<=1
$$

where TET, $\mathrm{n}$ and $\mathrm{T}$ are as in Equation 3-4.

\subsubsection{Number of Oscillations (NO)}

In addition to the duration of disturbance, reflected by the TETIndex described in Equation 3-5, the number of oscillations (NO), reflecting the stop-and-go maneuvers, was also defined in this study. An oscillation is defined as a deceleration phase followed by an acceleration phase. Stop-and-go traffic is the mechanism of traffic state transition to congestion and is related to traffic breakdown and instability (Zheng et al., 2010). An oscillation occurs when the speed of the follower vehicle is changing while the leader's 
speed does not. This study measured NO directly from individual's vehicles acceleration/deceleration. The percentage of vehicles having oscillation in each 300 feet segment is calculated and used as another disturbance indicator.

\subsubsection{Rear-End Crash Index (RCI)}

Rear-end crash Index (RCI) is another surrogate safety measure proposed based on the safe stopping distance in car following situations (Oh et al., 2006; Ozbay et al., 2007). Safe stopping distance is defined as that, at which the follower vehicle can safely reduce speed to avoid colliding with the leading vehicle when the leading vehicle reduces its speed or stops. Using the RCI, the judgment of whether a conflict occurs is based on the trajectories parameters of two consecutive vehicles including the relative speed, distance, and acceleration between the leading and following vehicles. RCI was defined as follows:

$$
\begin{aligned}
& \mathrm{RCI}=\left(\sum_{\mathrm{t}=1}^{\mathrm{T}} \mathrm{RC}(\mathrm{t})\right) / \mathrm{n} \\
& \mathrm{RC}(\mathrm{t})=\sum_{\mathrm{i}=1}^{\mathrm{n}} \mathrm{SDI} \times \Delta \mathrm{t} \\
& \mathrm{SDI}= \begin{cases}0 & (\text { safe }), \\
1 & (\text { unsafe }),\end{cases}
\end{aligned}
$$

where, $\mathrm{RC}(\mathrm{t})$ is the number of conflicts at time $\mathrm{t}, \Delta \mathrm{t}, \mathrm{t}, \mathrm{T}$ and $\mathrm{n}$ are as defined before and the stopping distance (SDI) is calculated as function of the reaction distance and braking distance as follows:

$$
\begin{aligned}
& \mathrm{SDF}=\mathrm{v}_{\mathrm{f}} \times \mathrm{PRT}+\frac{\mathrm{v}_{\mathrm{f}}{ }^{2}}{2 \times \mathrm{a}_{\mathrm{f}}} \\
& S D L=\mathrm{v}_{\mathrm{l}} \times \mathrm{PRT}+\frac{\mathrm{v}_{\mathrm{l}}^{2}}{2 \times \mathrm{a}_{\mathrm{l}}}+\overline{\mathrm{l}} \\
& \mathrm{SDL}_{\text {Break }}=\overline{\mathrm{l}} \\
& \overline{\mathrm{l}}=\mathrm{x}_{\mathrm{i}-1}(\mathrm{t})-\mathrm{x}_{\mathrm{i}}(\mathrm{t})-\overline{\mathrm{L}}
\end{aligned}
$$


where SDF is the stopping distance of the following vehicle, SDL is the stopping distance of the leading vehicle, PRT is the perception reaction time, set to 1.5 seconds (AASHTO, 2011), $a$ is deceleration rate, $v$ is the speed and $\bar{l}$ is relative distance. $x_{i}(t)$ and $\bar{L}$ are as

defined before. Please note that since this study uses $\mathrm{TTC}_{\mathrm{Break}}, \mathrm{SDL}_{\mathrm{Break}}$ was defined in this study to extend the original concept of SDL to reflect conditions, at which the leading vehicle stops suddenly causing a conflict with the lagging vehicle.

\subsection{Utilized Data}

A complete understanding of the traffic conditions will benefit from collecting the data at the individual vehicle level and generating time-space diagrams showing vehicle interactions, disturbances in traffic flow, congestion formation, and shockwave propagation that are important in assessing traffic safety and mobility. As stated earlier, the recent advancement of emerging technologies such as $\mathrm{CV}$ and $\mathrm{CAV}$ promise to provide such data in real-time. However, CV and CAV data are not yet available from traffic streams with high market penetrations of these technologies to allow its use in this study. The real-world trajectory data collected as part of the Federal Highway Administration (FHWA) by Next Generation SIMulation (NGSIM) program was also collected only for congested conditions, and thus does not fully satisfy the data requirements of this study. Therefore, simulation modeling was used to produce trajectory data for different congestion levels for use in the development of the models in this study. However, realworld vehicle level data in addition to macroscopic traffic data were used in calibrating the simulation model. In addition, vehicle-level real-world data from other locations were used in validating and evaluating the developed models, as described later in this chapter. 
Thus, the utilized data includes the following three groups:

- Simulated vehicle trajectory from microscopic simulation

- Real-world vehicle trajectory from NGSIM data

- CV data from real-world deployments of connected vehicles (CV)

The trajectory data from microscopic simulation, NGSIM data, and CV data include information about vehicles' speeds, accelerations/decelerations, and locations at the 1/10th of a second resolution. These trajectory data were associated with subsegments per lane, each measuring 300 feet long. The segment performance was assessed for each 5-minute interval, unless otherwise stated.

\subsubsection{Microscopic Simulation}

Simulation modeling of a freeway segment was performed utilizing the PTV's Verkehr In Städten SIMulationsmodell (VISSIM) microscopic simulation tool. The simulated segment includes an on-ramp and off-ramp with three main lanes throughout the section. The merge of the on-ramp traffic creates a bottleneck with different levels of congestion at the upstream section, depending on the traffic level. The segment was simulated and calibrated to reflect real-world conditions on the eastbound segment of the I-580 in California, between Strobridge Ave and Redwood Rd. The calibration process utilized data and results from a FHWA study, in which the time headways of all vehicles were collected utilizing a drone video recording and analyzed using an image processing software (Hale et al., 2019). The FHWA study utilized a previously developed method (Rakha \& Gao, 2010) to calibrate the Wiedemann model based on the collected headway data. Please refer to Hale et al. (2019) and Rakha \& Gao (2010) for the utilized VISSIM calibration processes and its validation. 
A one-mile segment in the simulation was divided into subsegments per lane, each is 300 feet long, to allow a detailed spatial analysis of the segment for each 5-minute time intervals for a total period of 15-minutes. The first 30 minutes of VISSIM simulation was set as a warm-up period and thus was excluded from the period of performance assessment. Figure 3-1 shows the coded freeway section.

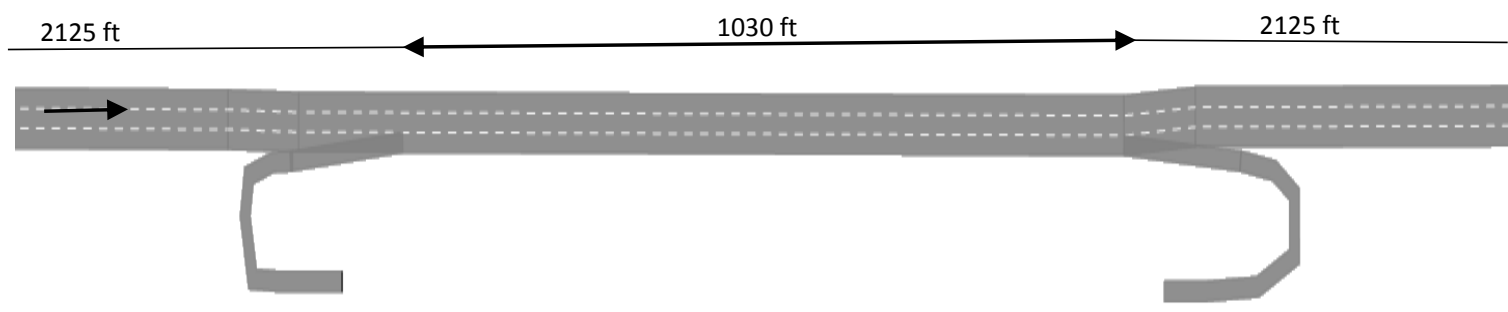

Figure 3-1 : Coded Freeway Section

\subsubsection{NGSIM Data}

The data from the simulation effort described above was used to derive a model to estimate safety and mobility performance. To validate the transferability of the model in producing acceptable results in other locations not used in the calibration of the simulation model, this study used real-world trajectories data collected from both I-80 and US 101 segment in California as part of the NGSIM program (FHWA, 2007) for the two locations. The segment of US101 is about 2,100 feet long and has five mainline lanes, one on-ramp and one off-ramp. The data were collected for the period between 7:50 A.M. to 8:35 A.M, for a total period of 45 minutes. The segment of I-80 is about 1,650 feet long and has five mainline lanes, one on-ramp, and one off-ramp. The I-80 data were collected for the period between 4:00 P.M. and 4:15 P.M. Figure 3-2 shows the study areas of I-80 and US101. 


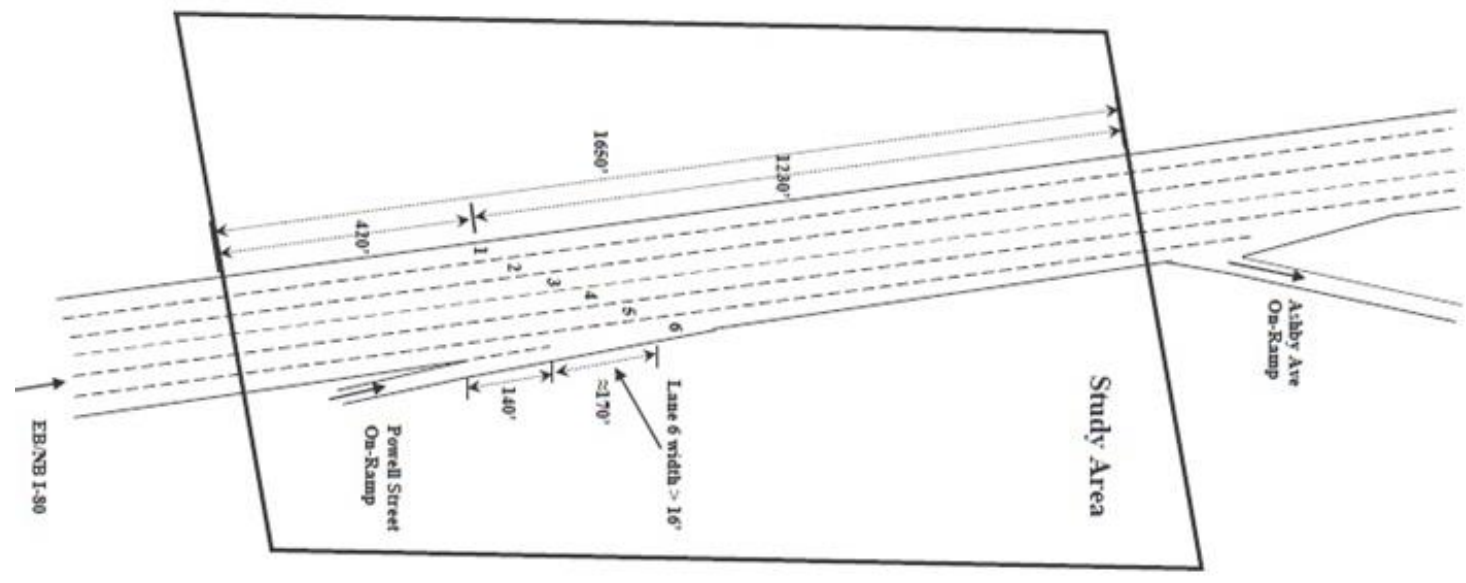

(a)

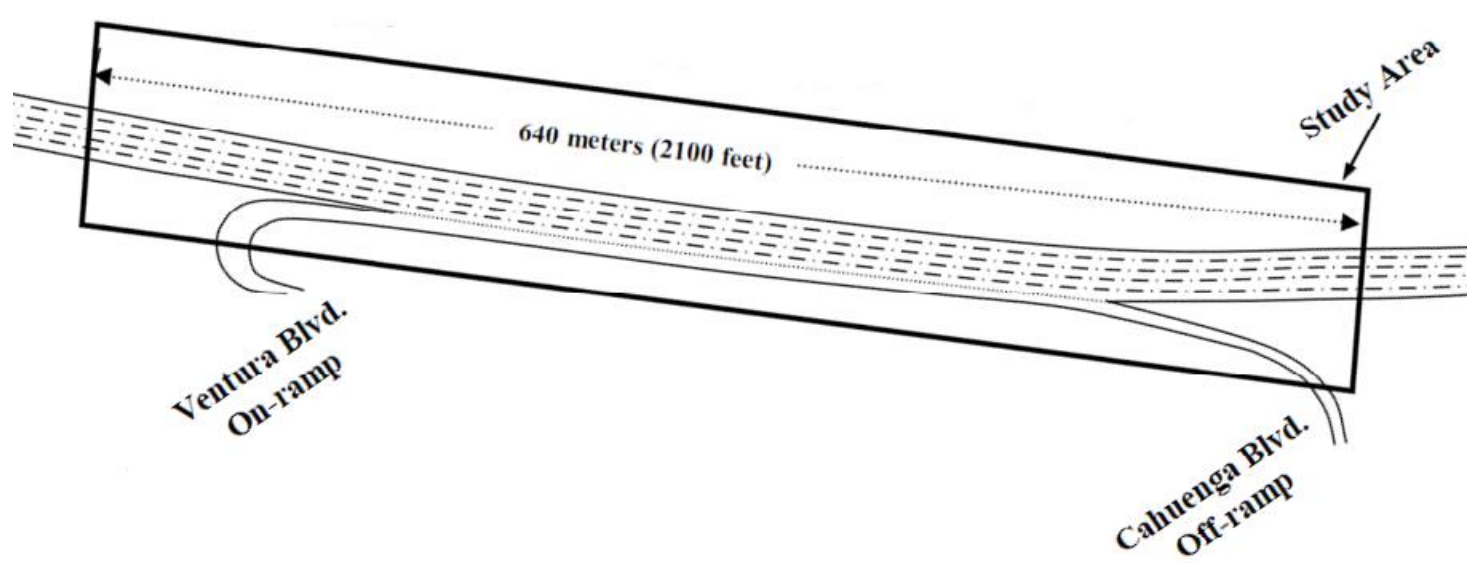

(b)

Figure 3-2: NGSIM Program Study Areas (a) I-80 and (b) US101

\subsubsection{Connected Vehicle Data}

Further evaluation of the application of the developed models for safety and breakdown prediction was done using data collected based on BSM from connected vehicles as part of the SPMD project in Ann Arbor, Michigan. The data was collected for 3,600 feet of the eastbound direction of I-94. This study used this data to assess the performance of the developed method when the data is obtained from low market 
penetrations of CV. The SPMD included about 2,800 instrumented vehicles and about 70 miles of roads instrumented with road side units (RSU) (Henclewood, et al., 2014). The CV and RSU communicated via DSRC communication. The selected segment has two lanes and located between ramps (Figure 3-3)

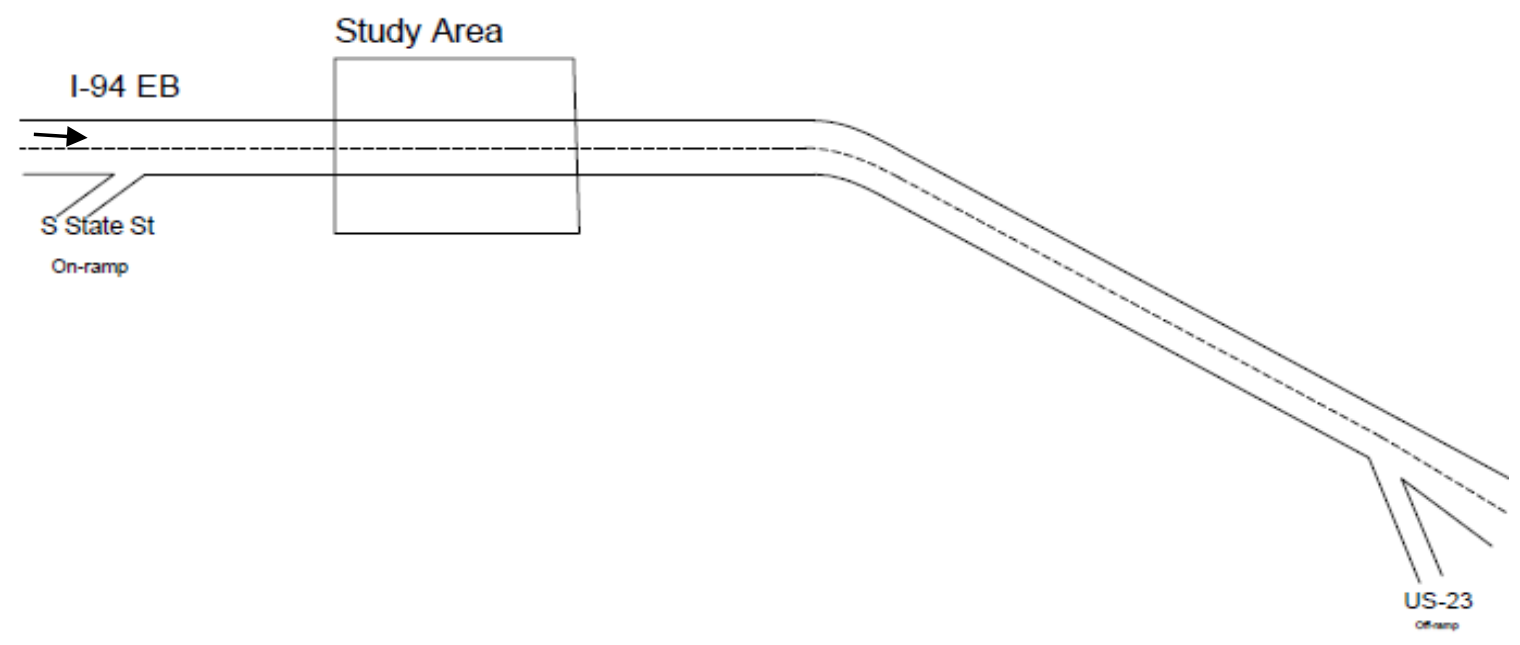

Figure 3-3: SPDM Study Area

\subsubsection{Descriptive Statistics of Utilized Data}

The summary statistics for utilized data including the simulated dataset, CV dataset, and NGSIM dataset are reported in Table 3-1. The features included in Table 3-1 are microscopic and macroscopic measures explained earlier in section 3.1. 
Table 3-1: Descriptive Statistics of Features

\begin{tabular}{|c|c|c|c|c|}
\hline \multicolumn{5}{|c|}{ Real-World CV Data from the SPMD Project and Detector Data (One day) } \\
\hline Features & Mean & Standard Deviation & Minimum & Maximum \\
\hline Mean Speed (fps) & 93.88 & 15.05 & 45.18 & 120.41 \\
\hline Avearge SDv (fps) & 0.88 & 0.74 & 0.04 & 4.08 \\
\hline SDt (fps) & 6.66 & 5.05 & 0.10 & 22.30 \\
\hline TETIndex & 0.03 & 0.02 & 0.01 & 0.15 \\
\hline NO $(\%)$ & 17.15 & 36.63 & 0.00 & 100.00 \\
\hline Flow (vphl) & 693.00 & 228.10 & 104.00 & 1814.00 \\
\hline Occupancy (\%) & 13.25 & 10.23 & 1.50 & 56.60 \\
\hline \multicolumn{5}{|c|}{ Simulated Data } \\
\hline Mean Speed (fps) & 73.83 & 25.60 & 12.20 & 106.34 \\
\hline Avearge SDv (fps) & 1.59 & 1.38 & 0.09 & 5.02 \\
\hline SDt (fps) & 5.95 & 3.53 & 1.91 & 17.87 \\
\hline TETIndex & 0.035 & 0.032 & 0.0013 & 0.17 \\
\hline NO (\%) & 16.28 & 24.23 & 0.00 & 100.00 \\
\hline Flow (vphl) & 1772.00 & 322.00 & 693.00 & 2488.00 \\
\hline Occupancy (\%) & 28.98 & 16.34 & 7.29 & 95.86 \\
\hline \multicolumn{5}{|c|}{ NGSIM : I-80 (4:00 P.M. and 4:15 P.M.) } \\
\hline Mean Speed (fps) & 25.76 & 4.39 & 15.69 & 37.20 \\
\hline Average SDv (fps) & 3.86 & 0.73 & 2.35 & 5.75 \\
\hline SDt (fps) & 6.74 & 1.62 & 4.05 & 12.90 \\
\hline TETIndex & 0.09 & 0.03 & 0.05 & 0.19 \\
\hline NO $(\%)$ & 96.28 & 5.51 & 76.47 & 100.00 \\
\hline Flow (vphl) & 1293.00 & 210.90 & 824.00 & 1941.00 \\
\hline Occupancy $(\%)$ & 40.84 & 9.44 & 25.08 & 81.53 \\
\hline \multicolumn{5}{|c|}{ NGSIM : US101 (7:50 A.M. to 8:35 A.M.) } \\
\hline Mean Speed (fps) & 34.25 & 5.08 & 24.08 & 45.15 \\
\hline Average SDv (fps) & 3.62 & 0.49 & 2.42 & 4.46 \\
\hline SDt (fps) & 9.88 & 1.72 & 6.70 & 14.28 \\
\hline TETIndex & 0.13 & 0.025 & 0.07 & 0.19 \\
\hline NO (\%) & 76.26 & 21.14 & 18.75 & 100.00 \\
\hline Flow (vphl) & 1677.00 & 134.20 & 1392.00 & 2004.00 \\
\hline Occupancy (\%) & 41.38 & 4.65 & 31.00 & 55.21 \\
\hline
\end{tabular}

\subsection{Platooning Measures}

One of the objectives of this dissertation is to estimate platooning measures at low market penetration of CV data. Several studies used time headway and its distribution as criteria to identify platooned vehicles. Although the headway distribution is a good measure to use in estimating the platooning characteristics, the data required for headway estimation will not be available based on data from low market penetrations of $\mathrm{CV}$ data, as 
described earlier. The headway measurement requires the location and speed of both the leading and lagging vehicles, and these vehicles will have to be equipped with $\mathrm{CV}$ technologies to provide this information. The headway data can be also obtained from connected automated vehicles if information from the vehicle sensors installed in these vehicles such as image-based sensors and microwave sensors are made available for performance measurement. However, this is not expected in the near future. Thus, measures other than time headway is needed to estimate platooning at lower CV market penetrations.

This section identifies methods for the estimation of the percentage of vehicles in the platoon and the distribution of the platoon size based on surrogate measures that can be assessed using CV data at a relatively low market penetration of connected vehicles. This study utilizes two surrogate measures for this purpose, the standard deviation of vehicles' speed (SDt), and the average of the standard deviations of individual vehicle's speeds (SDv) as were described before.

To investigate the relationship between the surrogate measures and the platooning characteristics, this study utilized real-world trajectory data collected as part of the Federal Highway Administration (FHWA) NGSIM program. Since this data was collected for congested conditions with high levels of platooning, trajectory data generated from simulation analysis were used to supplement the real-world data, which provided the coverage of additional platooning levels. The mean speed, SDv and SDt were calculated based on the real-world and simulated trajectories. A moving average method was used to remove the noise in the vehicle speed profile. All vehicles were sorted by their entrance 
times to the 300 feet subsegment and were assigned vehicle identifications for use in the determination of the platooning of vehicles.

Two criteria were used to decide whether a vehicle is platooned or not. The first criterion is the time headway. A time headway of 4.0 seconds was selected as a threshold to identify the platoon based on data analysis that involved plotting vehicle headway vs. speed, sensitivity analysis that involves varying the headway threshold between 3.0 seconds to 5.0 seconds, and findings based on the literature review. Since the standard deviation of speed of the leading vehicles should be small, another criterion was used to supplement the time headway criterion in the identification of a non-platooned vehicle or the leading vehicle of a platoon based on the standard deviations of individual vehicle speed. Based on examining the data used in this study and the review of literature, the standard deviation of speed below 1.0 fps was used as an indication of a non-platooned vehicle or the leading vehicle in a platoon, if the vehicle also meets the headway criterion. The reason for using $1.0 \mathrm{fps}$ is because in Equation 2-1, if the specified number of vehicles in a platoon is 1 , then the $\mathrm{SDv}$ is $0.26 \mathrm{~m} / \mathrm{s}(0.88 \mathrm{fps})$, which is close to $1 \mathrm{fps}$.

The above procedure allows the estimation of the percentage of vehicles in a platoon based on all available trajectories. In addition, the distribution of the size of the platoon was estimated by estimating the position of each vehicle in the platoon based on a model developed by Tian et al. (2017) that links the standard deviation of the speed of a vehicle to its position in the platoon. This model was previously presented in Equation 21. This relationship was validated based on the data collected in this study, as discussed later in this document. 
The above discussion summarizes the estimation of two platooning measures for use in this study: the percentage of vehicles in a platoon and the distribution of the size of the platoon. Since the purpose of this study is to link the two surrogate measures (SDv and SDt) to the platooning measures discussed above, the relationships between the surrogate measures and the platooning measures were then developed based on all available trajectories since the platooning measures can only be measured if all trajectories are used. The trajectories of all vehicles cannot be obtained unless the market penetration of $\mathrm{CV}$ is $100 \%$. Thus, the developed relationship was derived for use to estimate the platooning measures based on the surrogate measures for lower market penetrations.

As stated earlier, the trajectories utilized in this study were obtained from two sources: real-world trajectories collected from I-80 in Emeryville, California by the NGSIM program, and simulated trajectories for the same corridor. For both the real-world and simulation, the segment was divided into five subsegments per lane, each measuring 300 feet long. The platooning measures and surrogate measures were estimated for each subsegment per 15-minute time interval.

\subsection{Safety Measures}

Another objective of this study is to use trajectory data in estimating metrics of the safety of freeway segments using data collected from low market penetrations of CV. The safety performance was assessed based on indicators of the interactions between pairs of vehicles in the traffic stream. These interactions at the individual's vehicle level creates perturbation in the traffic flow that can potentially lead to traffic breakdown or collisions.

This study investigated methods for the utilization of detailed trajectory data to measure disturbances metrics as indictors of the perturbation of traffic flow to identify 
unsafe conditions. The utilized disturbance metrics are the number of oscillations (NO) and a measure of disturbance durations index based on time exposed time-to-collisions (TET), referred to as TETIndex. This study also measured NO directly from individual vehicles' acceleration/deceleration in transition to congested conditions. TET has been widely used as a safety surrogate measure, however, to the best of author's knowledge, there is no study on the identification of its thresholds to justify activating plans to mitigate unsafe conditions in real-time operations. TET also has not been sufficiently investigated as a realtime indicator of safety under different conditions and with smaller sample sizes of trajectory data. The TETIndex estimation requires the location and speed of both the leading and following vehicles and therefore cannot be measured accurately at low sample sizes of vehicle trajectories. Thus, this study derived regression models to estimate the TETIndex based on speed parameters for use in cases such as low market penetrations of $\mathrm{CV}$. The considered speed parameters are the mean speed, the average of the standard deviations of individual vehicle's speeds (SDv), and standard deviation of vehicles' speed (SDt). The developed model was tested using real-world trajectory data from two locations that were not used in the development of the model. The model was also used to estimate the TETIndex at low market penetrations of real-world vehicle trajectories based on CV data and the results were related to crash data at the site.

The disturbance metrics were also related to an additional surrogate measure of safety referred to the rear end crash index (RCI) to confirm the critical values of the disturbance metrics that indicate unsafe conditions, as described later. The parameters used in this research are described in the section 3.1. When calculating these parameters, a moving average method was used to remove the noise in the vehicle speed profile. The 
estimation was done for each 300 feet subsegment of the case study facility, per lane and for each 5-minute time intervals. Figure 3-4 presents an overview of the utilized data sources, the developed method, and the associated evaluation.

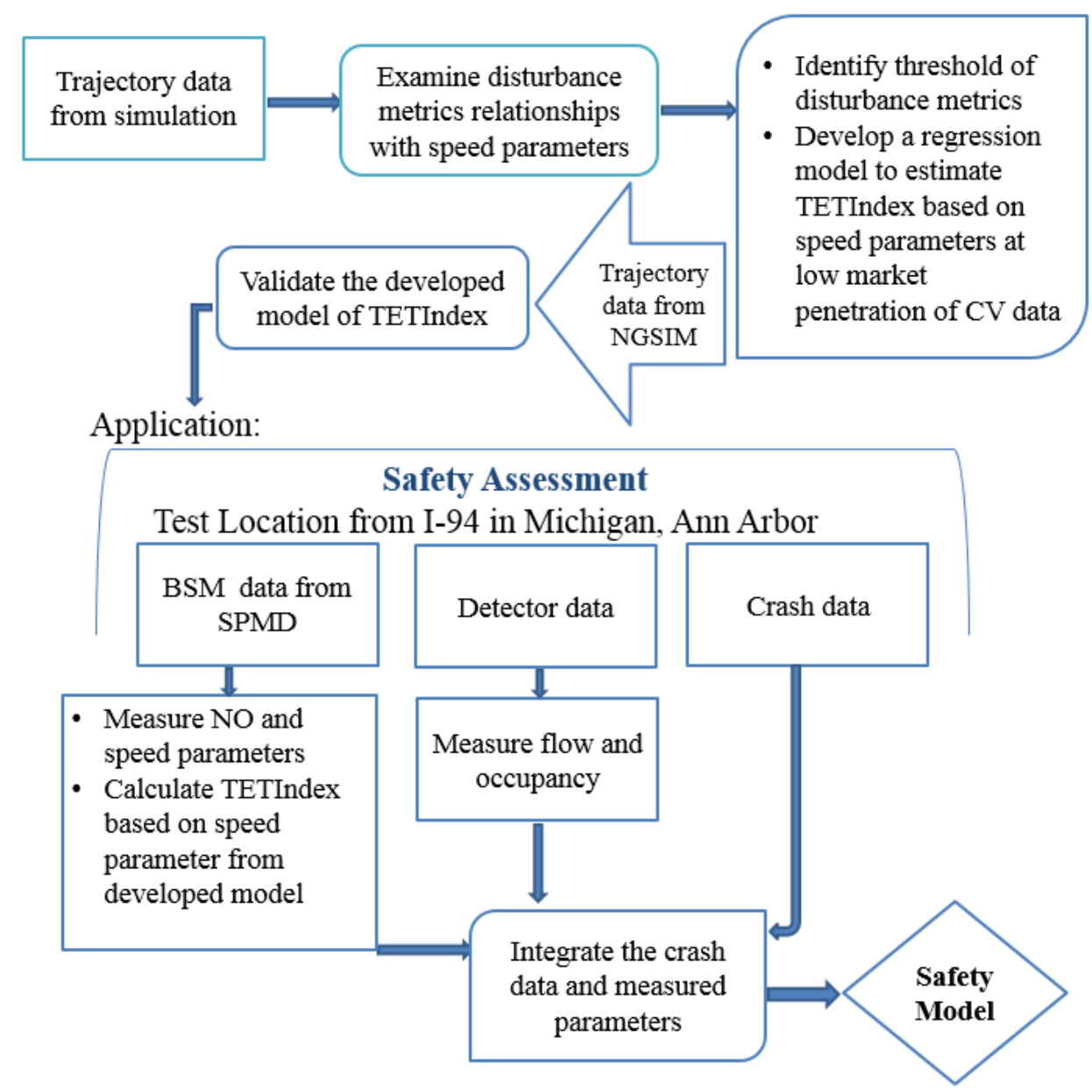

Figure 3-4: Methodology Framework of the Assessment of Safety

\subsection{Traffic State Identification}

This study investigated the use of microscopic features that contribute to traffic flow perturbations as indicators of breakdown, in addition to the commonly used macroscopic measures. Three methods were used and compared in identifying the breakdown state. The first and second methods use clustering technique and the change- 
point regression, respectively, based on macroscopic measures, as have been done in previous studies and discussed in the literature review. The third method, as a contribution of this study, uses a combination of macroscopic and microscopic features in the clustering analysis to identify the traffic state. The first step in the analysis was to identify the conditions, under which there is uncertainty in whether a breakdown has occurred when using the macroscopic measures alone. This was determined by using clustering based on the macroscopic measures and identifying the uncertain traffic conditions with high probabilities of belonging to two clusters, one cluster indicating a "breakdown" and one indicating "non-breakdown". The Gaussian Mixture Models (GMM) with the three macroscopic features of traffic (flow rate, speed and density) was used to identify the uncertain phase of traffic conditions. The reason for using the GMM clustering for this purpose instead of using other more commonly used clustering methods is that the GMM clustering is capable of providing the probability of belonging to the identified clusters (Bishop, 2006).

The identified uncertain traffic condition phase from the GMM analysis was then further analyzed using the K-Means clustering, with consideration of both macroscopic and microscopic features. The microscopic features include the standard deviation of speed, number of oscillation (stop-and-go), and TETIndex, which are indicators of disturbances. The results from using these additional microscopic features in the clustering are then evaluated and compared to those using only macroscopic measures. In addition, the performance of the method was compared with the results obtained from using a deterministic value of speed as an indicator of breakdown as identified using the change- 
point regression analysis procedure based on the speed-occupancy relationship. Finally, the method was tested using NGSIM dataset.

\section{Clustering Analysis:}

Clustering is the grouping of a set of data into clusters where the data in the same cluster are similar in some sense. There are several clustering methods that have been proposed and utilized in the literature. The two approaches mentioned earlier, the GMM and K-Means clustering methods have their own strengths and weakness. GMM is a probabilistic clustering that fits a set of number of Gaussians (number of components) to the data and estimates the Gaussian distribution parameters (the mean and variance) for each cluster and the size of a cluster. It then calculates the probability of the data points belonging to each cluster (Neal, 2007). The K-Means method clusters the data points based on the average squared distance between the points such that the distance in the same cluster is minimized (Arthur \& Vassilvitskii, 2007). The K-Means methods, which is the most widely used clustering method, clusters the data deterministically while in reality there might be some overlapping between the clusters. The GMM addresses this issue by providing the probability of a data point belonging to a specific cluster. However, the KMeans clustering was found to performs better with high dimensional data (Neal, 2007).

As mentioned earlier, there is uncertainty in the data belonging to clusters around the breakdown point. Thus, in this study a hybrid clustering approach that combines GMM and K-Means was used to better categorize the data around the breakdown point. The clustering algorithm and analysis were implemented using the scikit-learn library in Python. First, the GMM was used to identify the traffic phase with uncertainty where the probability of being in more than one cluster is significantly higher than zero. Then, the K- 
Means clustering was used to classify that data points in the phase of uncertain traffic conditions into "breakdown" and "non-breakdown" conditions based on different combinations of macroscopic and microscopic traffic features. Note that clustering the uncertain phase with GMM was also tried. It was found that the K-Means clustering outperformed the GMM with regard to the Silhouette Coefficient (SC), which is a measure used for clustering assessment, explained in the next paragraph.

Recognizing the difference in scale between the used features, the values of each feature were standardized before clustering by subtracting the mean and then dividing by the standard deviation of the feature values (Hale, 2018). The results of the clustering algorithm were assessed using the SC as a performance measure of clustering. The SC value for each point is a measure of how that point is similar to the points in its own cluster, compared to the points in another cluster. The value is between -1 and 1 . Values close to one indicate data that the data is very well clustered (Liu et al., 2016). In addition to the similarity of the points in the clusters, this value also increases with the decrease in the number of features. Thus, this should be considered when comparing the performance of different clustering alternatives using the SC.

\subsection{Traffic Flow Breakdown Prediction}

This study investigated the use of combinations of macroscopic and microscopic features to identify the mobility and safety state of freeway traffic. The investigated microscopic features included the standard deviations of individual vehicle's speeds and the standard deviation of vehicles' speed, in addition to the two disturbance metrics of traffic flow, mentioned earlier. The two disturbance metrics are the number of oscillations (stop-and-go) and a metric that has been widely used as a surrogate metric to safety, 
referred to as the time exposed time-to-collision (TET). These disturbance metrics are expected to be good indicators of the perturbation in traffic flow and also safety, thus allowing more accurate alerts to traffic management agencies regarding entering a nonacceptable traffic state. The developed and evaluated models predict the breakdown state in term of combined mobility and safety metrics in the next 5-minute interval in realtime operations. Three different machine learning approaches were used and evaluated for developing state prediction models using data from simulation modeling and data from a real-world CV deployment, as part of the SPMD.

\section{Utilized Machine Learning Approaches:}

Machine learning methods/algorithms are classified as supervised, unsupervised, and reinforcement learning. Supervised learning requires training data that include feeding paired inputs and outputs to the model. Examples of supervised learning are statistical regressions, K-nearest neighbors, support vector machine (SVM), artificial neural networks (ANN), decision trees, and tree ensembles. With unsupervised learning, the input data is not associate with outputs. Examples are clustering and association rules. Reinforcement learning can observe the conditions and select the best actions for a given situation (Bishop, 2006).

For the real-time classification of traffic state, as explained in previous sections, this study first categorized the historical traffic data using unsupervised clustering technique that separates the traffic states into "breakdown" and "non-breakdown", based on combinations of microscopic and macroscopic features, that reflect both mobility and safety. The identified traffic state was then used as a binary response label, to the data that 
is used in the training and testing of the machine learning classification methods used in this study.

The top ten classifiers that are widely used in research and practice were attempted using the scikit-learn library in Python. Each of these classifiers has its own weakness and strengths Based on the initial evaluation results, three of the classifiers were selected for use in this study. These selected classifiers are: the SVM and two tree ensemble techniques, which are the Random Forest (RF) and Extreme Gradient Boosting (XGB). RF is one of the simplest, popular and accurate learning algorithm and act excellent in prediction performance. RF trains each tree individually using a random feature. XGB, known as regularized boosting, is an implementation of gradient boosted decision trees designed for speed and performance. XGB builds each new tree correcting errors made by the previous trees. SVM is a linear/non-linear separator that transform the data and found the optimum boundary to separate the data into two classes (Bishop, 2006).

As stated earlier, several macroscopic and microscopic features were utilized as inputs to the investigated models. Recognizing the difference in scale between the used features, the values of each feature were standardized before clustering by subtracting the mean and then dividing by the standard deviation of the feature values. Since most features are correlated with each other, first, the Principal Component Analysis (PCA) was utilized to reduce the dimensionality of data and handle the collinearity between variables. The utilized number of components was selected based on the number of input features and projected variance (Bishop, 2006). 


\subsection{Summary}

This chapter discussed the methodologies and utilized data that were adopted to achieve the objectives of this research. Methodologies for platooning estimation, safety state estimation, traffic operation state recognition, and traffic flow breakdown prediction were discussed. Simulated trajectory data were used to develop the models and NGSIM data were used to validate the further developed model. Data from a connected vehicle pilot with low $\mathrm{CV}$ market penetration was also used to assess the developed models for safety estimation and breakdown prediction at low market penetrations of CV. 


\section{CHAPTER IV \\ ANALYSIS, RESULTS AND DISCUSSIONS}

This section presents the results and discussion of the development, validation, and evaluation of the models and methods developed in this study.

\subsection{Relationships between Parameters}

First, the study performed an initial exploration of the relationship between the three fundamental macroscopic variables (flow, density, and speed) for the simulated data as presented by the fundamental diagrams and shown in Figure 4-1. A Change-Point Regression with Gaussian Mixture analysis was conducted based on the data from the calibrated simulation model and the critical speed at capacity was found to be around 65 fps. This value was used in further analysis of the results, as described later.
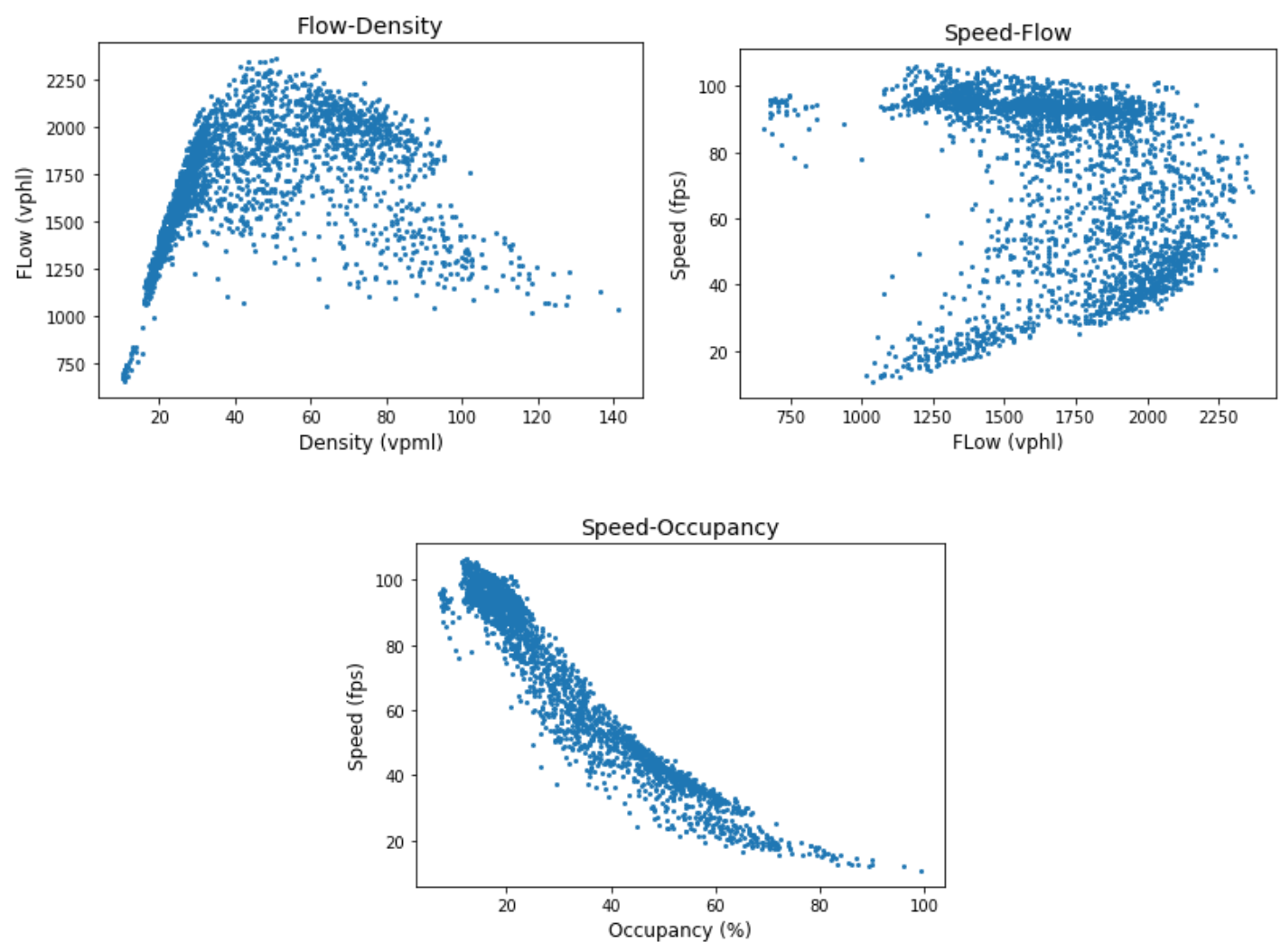

Figure 4-1: Fundamental Diagram of the Macroscopic Traffic Features 
Then, this study performed a visual inspection of the relationship between disturbance metrics (TETIndex and NO) with speed parameters (S, SDv and SDt), as described next.

Disturbance Metrics: TETIndex and NO Analysis:

The relationship between the speed parameters are shown in Figure 4-2. Figure 42 (a) shows that as the speed decreases, the SDv, increases reaching a maximum of about 4 fps at speed slightly below the critical speed at capacity (about $55 \mathrm{fps}$ which is lower than the speed at capacity, which is $65 \mathrm{fps})$. However, the SDv does not increase further when the speed decreased below 65 fps since the traffic is already in a congested regime. Figure 4-2 (b) shows that as the speed decreases, the SDt, increases reaching a maximum of about 8 fps around the breakdown point. As the traffic becomes more congested and the speed drops, the SDt decreases due to the constraint on the desired speeds of vehicle by their leaders.

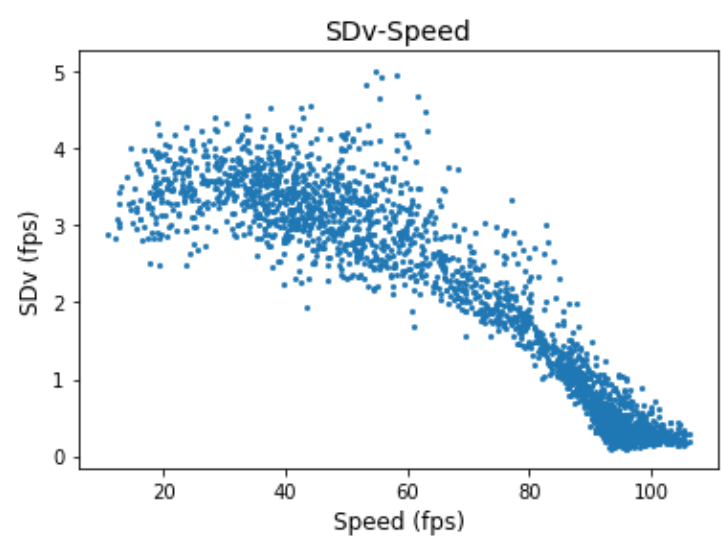

(a)

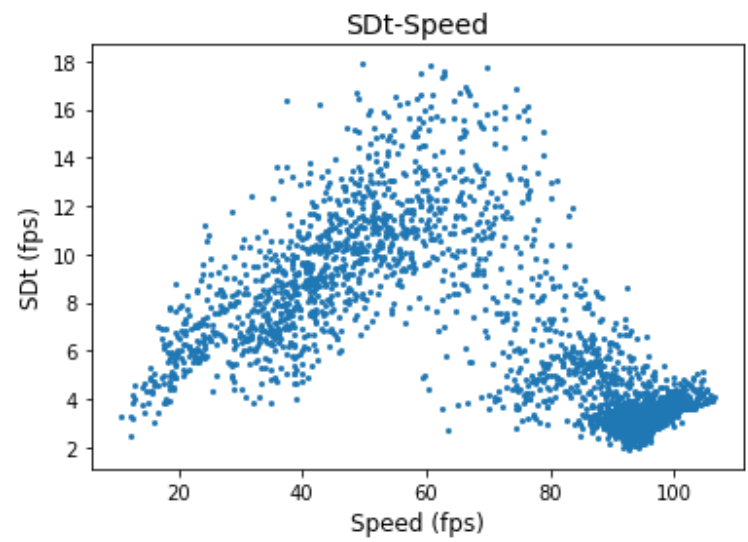

(b)

Figure 4-2: The Relationship between Space Mean Speed and (a) SDv and (b) SDt 
Visual inspection of the relationships of the two disturbance metrics (TETIndex and NO) and the speed parameters are shown in Figure 4-3. It can be seen that the TETIndex and NO increase with the decrease in the mean speed. They also increase with increase in SDv. In addition, the TETIndex and NO increase sharply as the speed drops below 65 fps and SDv increases above 2.0-2.5 fps. The values of the TETIndex and NO at the break point, beyond which the two values of the two variables increase sharply, are about 0.03 and $10 \%$, respectively. Figure $4-3$ shows that the values of the TETIndex and NO with the maximum SDt are also around 0.03 and $10 \%$, respectively. These values seem to indicate the start of the transition phase of traffic conditions, at which the perturbation of traffic flow is more likely to grow. Figure 4-3 shows higher values of the disturbance metrics including a TETindex of 0.05 and a $\mathrm{NO}$ of $20 \%$ are clearly in the congestion region of the curves.

\subsection{Platooning Measures}

Next, this study investigated the $\mathrm{CV}$ use of data at a relatively low market penetration of $\mathrm{CV}$ for platooning measure estimation including the percentage of vehicles in the platoon and the distribution of the platoon size based on surrogate measures. This study utilized two surrogate measures for this purpose, the standard deviation of vehicles' speed, and the average of the standard deviations of individual vehicle's speeds. 

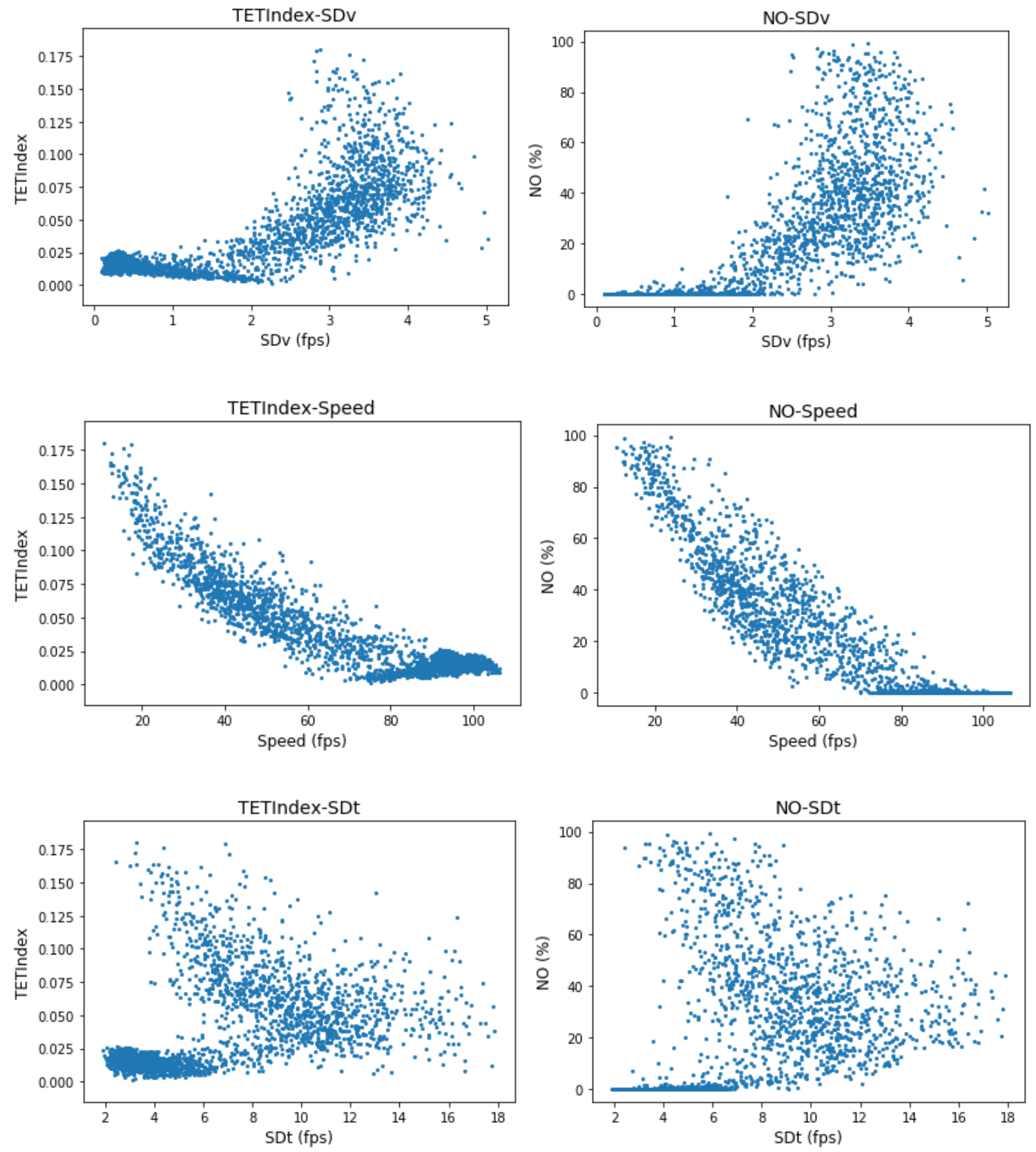

Figure 4-3: The Relationship between Disturbances with Mean Speed, SDv and SDt

\subsubsection{Platoon Percentage Determination}

As described earlier, the number of vehicles in the platoon was calculated in previous studies by identifying whether or not a vehicle is platooned based on the measured time headways and the standard deviations of individual vehicle's speeds based on vehicle 
trajectories. The result was used to calculate the percentage of the vehicles in the platoon. This calculation is possible only if $100 \%$ of the vehicle trajectories are available. Thus, the standard deviation of vehicles' speed (SDt), which can be measured at low CV market penetrations, was also measured based on the trajectories for potential use to estimate the percentage of vehicles in the platoon at low market penetrations. A regression analysis was conducted to derive the relationship between the percentage of vehicles in platoon for each subsegment and time interval and the corresponding SDt.

The analysis of the results shows that at low volumes, when the segment is operating at Level of Service C to D or better, according to the HCM procedure, the SDt does not change with the increase in demand and the percentage in platoons, as shown in Figure 4-1. However, an increase in the SDt is observed with a decrease in speed as traffic approaches breakdown, reaching a maximum close to traffic breakdown, as shown in Figure 4-2. Figure 4-2 shows that the maximum observed SDt is at a speed around $59 \mathrm{fps}$ (about $41 \mathrm{mph}$ ), which is beyond the critical speed at capacity estimated, which according to the HCM procedure, is around $77 \mathrm{fps}(52 \mathrm{mph})$. Around that point, which corresponds to about $84 \%$ of vehicles in the platoon, a significant increase in the SDt is observed, as shown in Figure 4-4. This can be explained by the fact that some vehicles may still be traveling at a relatively higher speed when they are not in platoons, while others are constrained due the following of slower vehicles in the platoons.

Beyond the point mentioned above ( $84 \%$ platoon percent and $40 \mathrm{mph}$ speed), as the speed decreases further and the platooning percentage increases, the relationship between the percent of vehicles in the platoon and SDt follows a negative log linear relationship, as shown in Figure 4-4. The decrease in the SDt with the increase in platoon 
percentage, and thus congestion, is expected since the increase in platooning after breakdown will further reduce the ability of vehicles to travel at their desired speeds. As shown in Figure 4-4 and Figure 4-5, SDt decreases from 12 fps when $84 \%$ of the vehicles were in the platoon and the average speed was $40 \mathrm{mph}$ to $6 \mathrm{fps}$ at a speed of $20 \mathrm{fps}$ (13 $\mathrm{mph}$ ) and $95 \%$ or more of the vehicles in platoon. It should also be mentioned that a direct relationship has also been found between the SDt and safety in previous work. Thus, the discussed relationship may be used to further assess the impacts of different levels of platooning on safety, in addition to mobility.

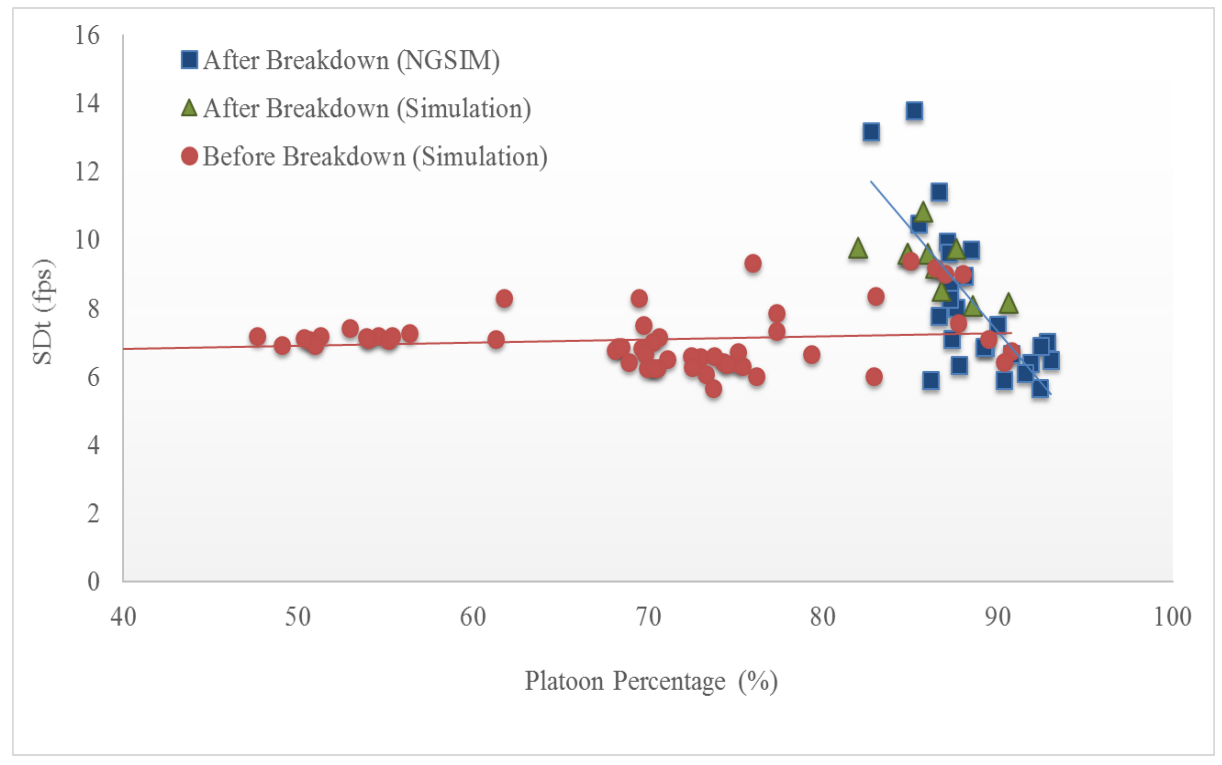

Figure 4-4: The Relationship between SDt and The Percentage of Vehicles in the Platoon 


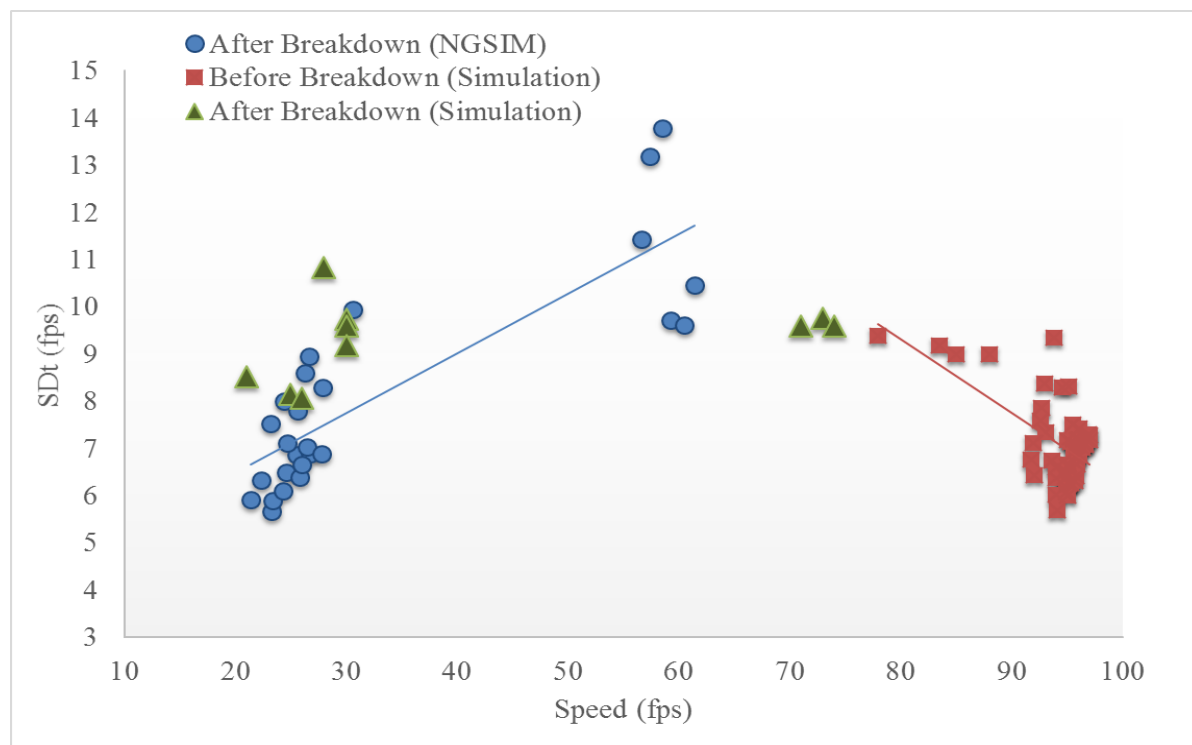

Figure 4-5: The Relationship between SDt and The Space Mean Speed

The fitted function between the percent of vehicles in the platoon and SDt for "after traffic breakdown" is provided in Equation 4-1.

$$
\mathrm{P}=\alpha+\beta \log (\mathrm{SDt})
$$

where $\mathrm{P}$ is the Percentage in the Platoon in the "After Breakdown" conditions, SDt is the standard deviation of vehicles' speed (fps), and $\alpha$ and $\beta$ are coefficients. The statistical software $\mathrm{R}$ was used to fit the regression equations and produce the statistics required to assess the significance of the relationships. The regression analysis results are presented in Table 4-1. Various transition forms were investigated, and the best form was selected. In addition to the statistical test results presented in Table 4-1, the model was also validated using the residual plot and the quantile-quantile (q-q) plot.

Table 4-1: Developed Equations to Estimate the Percent in Platoon

\begin{tabular}{|l|c|c|l|l|l|l|l|}
\hline Equation & $\alpha$ & $\beta$ & $\begin{array}{l}\text { Squared } \\
\text { Value }\end{array}$ & $\begin{array}{l}\text { Adjusted } \\
\mathrm{R}- \\
\text { Squared }\end{array}$ & $\begin{array}{l}\mathrm{t} \text { value of } \\
\alpha(\operatorname{Pr}(>|\mathrm{t}|))\end{array}$ & $\begin{array}{l}\mathrm{t} \text { value of } \beta \\
(\operatorname{Pr}(>|\mathrm{t}|))\end{array}$ & $\begin{array}{l}\text { Residual } \\
(\mathrm{P} \text {-value })\end{array}$ \\
\hline $\begin{array}{l}\text { After } \\
\text { Breakdown }\end{array}$ & 111.09 & -10.67 & 0.6354 & 0.625 & $\begin{array}{l}38.565 \\
(2 \mathrm{e}-16)\end{array}$ & $\begin{array}{l}-7.811 \\
(3.55 \mathrm{e}-09)\end{array}$ & $3.547 \mathrm{e}-09$ \\
\hline
\end{tabular}


Equation 4-1 can be used to estimate the percent of vehicles in platoons during breakdown conditions that appear to occur when $84 \%$ of the vehicles or more are in the platoon and the speed is below the estimated speed at capacity. The equation can be used to estimate the percent of vehicles in the platoon as a function of SDt when these estimates are made based on data from a low market penetration of connected vehicles. This is because the platooning characteristics cannot be measured directly at low CV market penetrations. In this study, the accuracy of this estimation was assessed at market penetrations of $5 \%, 10 \%, 50 \%$ and $80 \%$. For each market penetration, the percent of vehicles in the platoon was calculated based on the measured standard deviation of vehicles' speed. The assessment was performed utilizing the NGSIM data for the I-80 segment described previously. The vehicles with connected vehicle equipment for each CV penetration were selected randomly from all vehicles in the traffic stream. However, the accuracy of the estimation of the speed standard deviation is expected to depend on this selection when there is a high variation in the speed characteristics of the vehicles in the link, particularly at low market penetrations. Thus, a Monte Carlo analysis was used in this study to account for this stochasticity by randomly selecting different $\mathrm{CV}$ vehicles from the traffic stream for each Monte Carlo run. Twenty Monte Carlo runs were conducted, and the estimates of the percent of vehicles in the platoon were estimated using Equation 4-1, based on the SDt generated from these runs, and were then compared with those actually measured using vehicle trajectories from a $100 \%$ market penetration of CV. The Mean Absolute Percent Error (MAPE) and Standard Deviation of Percentage Error (SDPE) provide expressions of the measures used to assess the quality of the estimation as follows: 


$$
\begin{aligned}
& \text { MAPE }=\frac{1}{n} \sum_{i=1}^{n}\left|\frac{y_{i}-y}{y_{i}}\right| \\
& \text { SDPE }=\sqrt{\frac{1}{n-1} \sum_{i=1}^{n}\left(w_{i}^{2}-n \bar{w}^{2}\right)}
\end{aligned}
$$

where $w_{i}=\frac{y_{i}-y}{y_{i}}, y_{i}$ is the estimated value of the ith run, $y$ is the value at $100 \% \mathrm{MP}, \mathrm{n}$ is the total number of runs $(n=20)$, and $\bar{w}$ is the average of all the $w_{\mathrm{i}}$. Different quality measurements have different significance. SDPE is a measure of reliability of the estimates. MAPE is the average error of all runs. The results of the quality measure calculations are shown in Table 4-2. As can be seen from the results in Tables 4-2, the error in the estimation of the platoon percentage is low. Thus, it can be concluded that the platoon percentage can be estimated at the low market penetration of CV data accurately based on the methodology presented in this study.

Table 4-2: The Quality of the Estimation of the Percentage of Vehicles in Platoon with Different Market Penetrations

\begin{tabular}{|l|c|c|c|c|}
\hline \multirow{2}{*}{$\begin{array}{l}\text { Accuracy } \\
\text { Measure }\end{array}$} & \multicolumn{4}{|c|}{ Market Penetration } \\
\cline { 2 - 5 } MAPE & $5 \%$ & $10 \%$ & $50 \%$ & $80 \%$ \\
\hline SDPE & 1.9010 & 1.0423 & 0.2968 & 0.1882 \\
\hline
\end{tabular}

Sensitivity Analysis on the Utilized Time Headway Threshold

An analysis was conducted to determine the sensitivity of the platoon percentage in this study for the utilized time headway threshold. Figure 4-6 shows how the estimated percentage of vehicles in a platoon in the NGSIM data varies as the standard deviation of vehicles' speed changes with different time headway thresholds (5-second, 4-second and 3-second time headways). 


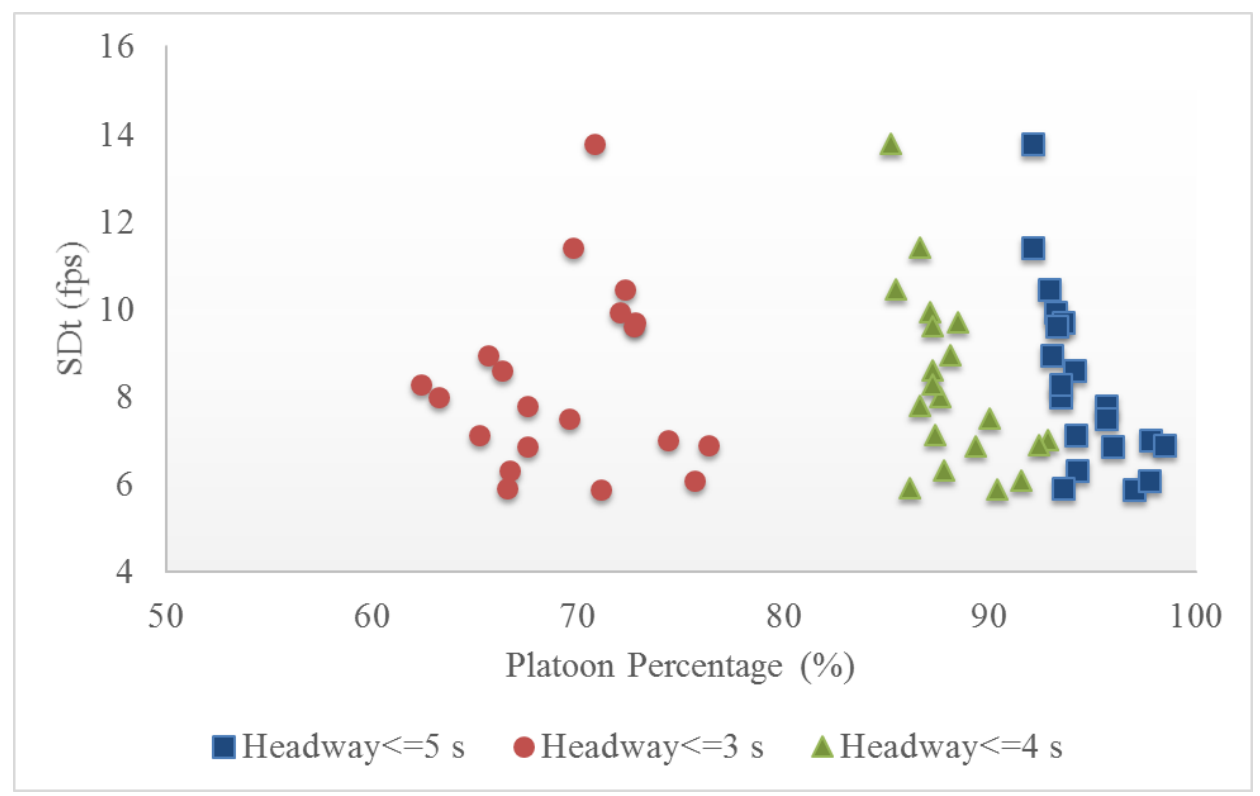

Figure 4-6: Platoon Percentages Estimated Using Different Headway Thresholds

As can be seen from Figure 4-6, the use of a 3-second headway as a threshold results in a lower percentage of vehicles in platoon and the use of a 5-second headway results in a higher percentage of vehicles in platoon than the 4-second headway. During a traffic congestion period, only $63 \%$ to $76 \%$ of the vehicles were identified as platooning when using the 3-second headway criterion. This indicates that using the 3 -second headway underestimate the percentage of vehicles in platoon. When the 5-second headway is used as the threshold, $100 \%$ of vehicles were identified as platooned. However, examining the standard deviations of individual vehicle's speeds shows that there are non-platooned vehicles, indicating that the 5-second threshold possibly overestimate the platooned vehicles percentage. Thus, the 4-second headway is used as the threshold in this study. 


\subsubsection{Determination of Platoon Size Distribution}

In addition to the percent of vehicles in the platoon discussed in the previous section, this study also investigates estimating the platoon size distribution based on the standard deviations of individual vehicle's speeds (SDv) at different market penetrations. The estimation is conducted for the "after breakdown." conditions Each non-platooned vehicle is considered a special vehicle platoon with platoon size equal to one. Tian et al. (2017) developed an equation to estimate the platoon size based on the standard deviations of individual vehicle's speed using NGSIM data for the U.S. 101 Freeway in California. In this study, we obtained the standard deviations of speed for several vehicles with different positions in the platoons utilizing NGSIM I-80 data. It was determined that the equation developed by Tian et al. (2017) produces reasonable results for the purpose of this research. This equation, as presented in the review of literature in Equation 2-1, was utilized in this study and rearranged to calculate the size of the platoon as a function of the SDv, as follows:

$$
\mathrm{NVP}=-94.29\left(\ln \left(10.56-0.3048 \times \mathrm{SD}_{\mathrm{v}}\right)-\ln (10.4)\right)
$$

where SDv is the average of standard deviations of individual vehicle's speeds (fps), and NPV is the number of vehicles in the platoon. The position of each vehicle in the platoon was estimated based on Equation 4-4 and with different CV market penetrations. The estimated positions were categorized into four groups, as shown in Table 4-3. The results in Table 4-3 are based on the full set of trajectories of the I-80 NGSIM data in a peak period from 4:00 p.m. to 4:15 p.m. 
Table 4-3: Platoon Size Percentage based on All Trajectories

\begin{tabular}{|c|c|c|c|c|}
\hline \multirow{2}{*}{ Subsegment } & \multicolumn{4}{|c|}{ Percentage of Vehicles in Platoon Size Group } \\
\cline { 2 - 5 } & $1<=\mathrm{x}<=2$ & $2<\mathrm{x}<=5$ & $5<\mathrm{x}<=15$ & $\mathrm{x}>15$ \\
\hline 1 & 0.75 & 13.43 & 57.09 & 28.73 \\
\hline 2 & 3.14 & 28.63 & 60.39 & 7.84 \\
\hline 3 & 3.82 & 32.44 & 56.87 & 6.87 \\
\hline 4 & 2.27 & 25.38 & 57.58 & 14.77 \\
\hline 5 & 4.60 & 24.52 & 53.64 & 17.24 \\
\hline
\end{tabular}

The percentage of vehicles in each platoon size group was estimated utilizing data that assumed different market penetrations of CV. The accuracy of this estimation was assessed at market penetrations of $5 \%, 10 \%, 20 \%, 50 \%$ and $80 \%$, compared to the base value of the comparison, which is the estimation when utilizing data from CVs at $100 \%$ market penetration. Twenty Monte Carlo runs were conducted to account for the stochasticity due to the random selection of different $\mathrm{CV}$ vehicles from the traffic stream, as discussed earlier. Each of these runs represents a different day of operations with different vehicles selected to be equipped with CV technology for each day.

The t-test of the difference in the mean and the Chi-square $\left(\chi^{2}\right)$ on the difference in the frequency distribution tests (McShane, 2011) were conducted to assess the accuracy of the estimated platoon size mean and distribution, respectively. For example, Table 4-4 shows the t-test and $\chi^{2}$ results for different market penetrations for one of the test's subsegments. The results of the t-test on the difference in the mean and the $\chi 2$ test of the platoon size distribution based on the mean of the 20 runs can be used to assess the adequacy of the estimation for planning purposes. The data used are average values based on data from 20 days, represented by the 20 runs. The results of the $\chi 2$ for each of the 20 days (runs) represent the adequacy of the estimation for use in the real-time operation for that single day. 
Table 4-4: t-Test and $\chi 2$ Test for Different Market Penetration Levels

\begin{tabular}{|c|c|c|c|c|}
\hline Hypothesis Test & \multicolumn{2}{|c|}{$\begin{array}{c}\text { Chi-Square Test on Frequency Distribution } \\
(\mathrm{df}=3)\end{array}$} & \multicolumn{2}{c|}{$\begin{array}{c}\text { t-test on the Mean } \\
(\mathrm{df}=19)\end{array}$} \\
\hline $\begin{array}{c}\text { Market } \\
\text { Penetration }\end{array}$ & $\begin{array}{c}\text { Is the Average of the } \\
\text { Runs passing Test } \\
\text { (Do not Reject Null } \\
\text { Hypothesis) }\end{array}$ & $\begin{array}{c}\text { No. of Individual Runs } \\
\text { Passing } \\
\text { (Do not Reject Null } \\
\text { Hypothesis) }\end{array}$ & $\begin{array}{c}\text { Do not } \\
\text { t-value } \\
\text { Hypothesis }\end{array}$ \\
\hline $5 \%$ & $\mathrm{Y}$ & 10 out of 20 & 1.013 & $\mathrm{Y}$ \\
\hline $10 \%$ & $\mathrm{Y}$ & 14 out of 20 & 0.5239 & $\mathrm{Y}$ \\
\hline $20 \%$ & $\mathrm{Y}$ & 18 out of 20 & 0.6665 & $\mathrm{Y}$ \\
\hline $50 \%$ & $\mathrm{Y}$ & 20 0ut of 20 & 1.3209 & $\mathrm{Y}$ \\
\hline
\end{tabular}

As can be seen from Table 4-4, when comparing the results from using the data at the $5 \%$ and $10 \%$ market penetration levels and using the data at the $100 \%$ level, the null hypothesis of no difference between the estimation of the means according to the t-test and no difference in the frequency distribution of the platoon size based on the average of the runs could not be rejected at the $95 \%$ confidence level. However, when considering the platoon size distribution for individual days, the null hypothesis can be rejected for 10 of the 20 days with a $5 \%$ market penetration, and 6 of the 20 days with a $10 \%$ market penetration at the $95 \%$ confidence level, indicating that the estimate is not adequate for operations at the 5\% and $10 \%$ market penetration levels. However, at a higher market penetration of $20 \%$, the null hypothesis of the $\chi 2$ test can be rejected for most of the individual runs (days) at the $95 \%$ confidence level. With this CV market penetration (20\%), the estimates can be used for both planning (based on the average of the runs) and operations (based on individual runs). Table 4-5 shows a comparison of the platoon size distribution using the data from each of the runs of the Monte Carlo simulation with a 20\% market penetration, the average of the runs with a $20 \%$ market penetration, and the distribution based on a $100 \%$ market penetration of CV. Inspection of the data in this table 
confirms that most Monte Carlo runs with the $20 \% \mathrm{CV}$ market penetration produces results that are comparable to those obtained with the $100 \%$ market penetration.

Table 4-5: Comparison of the Platoon Size Distribution Estimated with the 20\% and $100 \%$ CV Market Penetration Levels

\begin{tabular}{|c|c|c|c|c|c|c|}
\hline $\begin{array}{c}\text { Market } \\
\text { Penetration }\end{array}$ & & \multicolumn{4}{|c|}{ Percentage of Vehicles in Platoon Size Group } & \multirow[b]{2}{*}{ Mean of value } \\
\hline \multirow{21}{*}{$20 \%$} & Run & $1<=\mathrm{x}<=2$ & $2<x<=5$ & $5<x<=15$ & $15<x<=30$ & \\
\hline & 1 & 0.00 & 3.77 & 69.81 & 26.42 & 13.06 \\
\hline & 2 & 3.77 & 13.21 & 64.15 & 18.87 & 11.18 \\
\hline & 3 & 1.92 & 7.69 & 65.38 & 25.00 & 12.46 \\
\hline & 4 & 0.00 & 13.21 & 47.17 & 39.62 & 14.09 \\
\hline & 5 & 1.89 & 15.09 & 56.60 & 26.42 & 12.16 \\
\hline & 6 & 0.00 & 9.43 & 62.26 & 28.30 & 12.92 \\
\hline & 7 & 1.89 & 9.43 & 58.49 & 30.19 & 13.00 \\
\hline & 8 & 0.00 & 13.21 & 60.38 & 26.42 & 12.44 \\
\hline & 9 & 1.89 & 15.09 & 52.83 & 30.19 & 12.63 \\
\hline & 10 & 0.00 & 13.21 & 66.04 & 20.75 & 11.74 \\
\hline & 11 & 0.00 & 18.87 & 49.06 & 32.08 & 12.78 \\
\hline & 12 & 0.00 & 16.98 & 50.94 & 32.08 & 12.91 \\
\hline & 13 & 1.89 & 11.32 & 54.72 & 32.08 & 13.11 \\
\hline & 14 & 1.89 & 11.32 & 56.60 & 30.19 & 12.88 \\
\hline & 15 & 1.89 & 16.98 & 50.94 & 30.19 & 12.51 \\
\hline & 16 & 0.00 & 13.21 & 56.60 & 30.19 & 12.92 \\
\hline & 17 & 0.00 & 20.75 & 50.94 & 28.30 & 12.19 \\
\hline & 18 & 0.00 & 13.21 & 58.49 & 28.30 & 12.68 \\
\hline & 19 & 0.00 & 7.55 & 54.72 & 37.74 & 14.23 \\
\hline & 20 & 0.00 & 9.43 & 60.38 & 30.19 & 13.16 \\
\hline \multicolumn{2}{|l|}{ Mean } & 0.85 & 12.65 & 57.33 & 29.17 & 12.75 \\
\hline \multicolumn{2}{|l|}{$100 \%$} & 0.75 & 13.43 & 57.09 & 28.73 & 12.65 \\
\hline
\end{tabular}

\subsubsection{Relationship between Platooning and Disturbance Metrics}

This section discusses the relationships between platooning measures derived as expressed above and the disturbances metrics utilized in this study. The number of vehicles in platoon (NVP) was calculated based on Equation 4-4. The relationships between NVP and disturbance metrics were then explored as displayed in Figure 4-7. 

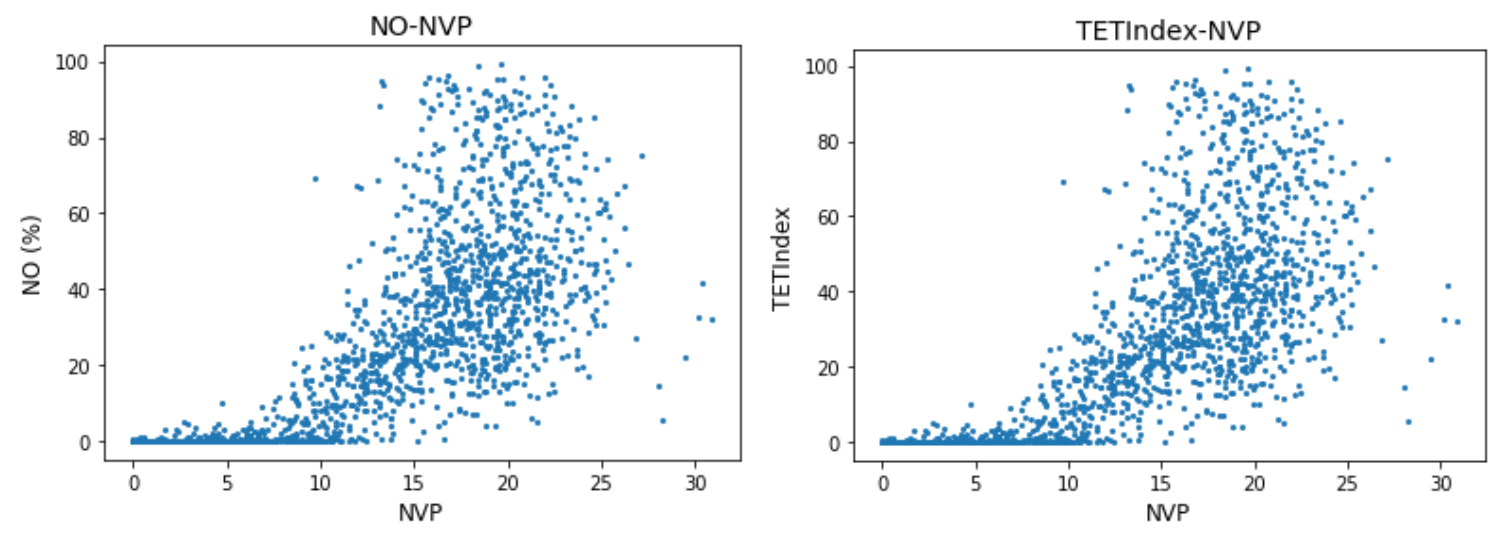

Figure 4-7: Relationship between Disturbance Metrics with NVP

As can be seen from Figure 4-7, the NVP increases after NO of $10 \%$ and TETIndex of 0.03. As stated earlier, the values of the TETIndex and NO also start increasing sharply at NO greater than $10 \%$ and TETIndex higher than 0.03 . The NPV is greater than 15 when the NO exceeds $20 \%$ and TETIndex exceeds 0.05 , which are the values previously reported where the traffic condition is in congested region. These results are consistent with founding in section 4.1 .

\subsection{Safety Measures}

Real-time safety assessment can be used as an important input to traffic management and operations. It is envisioned that when a threshold value of the estimated TETIndex and NO as a surrogate measures to safety are exceeded, a central decision support system (DSS) will recommend the activation of operational plans on the freeway such as metering and/or dynamic speed limit to reduce the probability of crashes. Thus, there is a need to identify the thresholds for these parameters that justify the activation of the plans. For this identification, this study uses a visual inspection of the graphical views of the relationships between the disturbance metrics and the speed parameters and RCI. The relationship between the speed parameters are shown in Figure 4-2. Visual inspection 
of the relationships of the two disturbance metrics (TETIndex and NO) and the speed parameters are shown in Figure 4-3. As it was explained in the section of 4.1, The values of the TETIndex and NO at the break point beyond which the two values of the two variables increase sharply are about 0.03 and $10 \%$, respectively. Figure $4-3$ shows that higher values of the disturbance metrics including a TETindex of 0.05 and a NO of $20 \%$ are clearly in the congestion region of the curves. Further inspection was done by examining the relationship between the TETIndex and NO and a third surrogate measure to safety, the RCI plotted in Figure 4-8. According to Oh et al (Oh et al., 2006), RCI values less than 0.2 can be considered as acceptable from rear-end crash risk point of view. Utilizing RCI value of 0.2 as reference, Figure 4-8 shows that this value is associated with TETIndex less than 0.03 and NO less than $10 \%$ and thus has an acceptable rear-end crash risk. Thus, based on the above, it was decided to use TETIndex of 0.03 and NO of $10 \%$ in this study, as the thresholds to determine unsafe conditions and potentially activate strategies to mitigate these conditions.

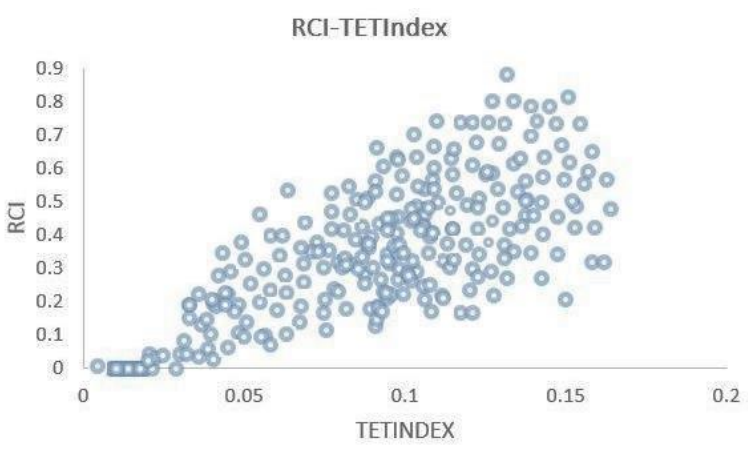

(a)

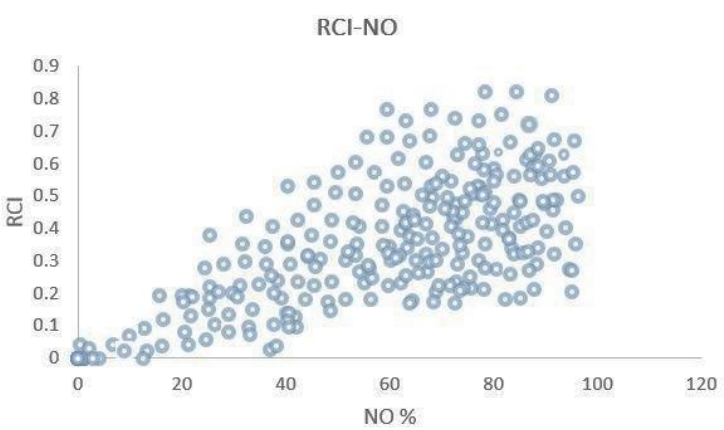

(b)

Figure 4-8: The Relationship between RCI and (a) TETIndex and (b) NO 


\subsubsection{Developying Model to Estimate TETindex at Low Market Penetration of CV Data}

As mentioned earlier, the TETIndex is not obtainable from small samples of data. Thus, regression analyses were conducted to derive the relationship between the TETIndex as the dependent variable and speed, SDv, and SDt, as the independent variables based on the full set of simulation results. The first step was to examine any collinearity in the data by producing the correlation matrix among the three variables. In addition, factor analysis was used to investigate how the variable values cluster to eliminate the impacts of independent variables correlating strongly with each other without significantly deteriorating the model fit of the data. Based on this analysis, it was determined that due to the relatively high correlation, the SDv variable can be either eliminated or respecified by utilizing a new variable that is a function of SDv (Johnson \& Wichern, 2007). The respecification was done by dividing SDv by speed. The resulting new variable was then used as an independent variable. This new variable is actually the coefficient of variation of the speed of individual vehicles. Several linear and non- linear functions between the TETIndex, Mean Speed (S), SDv and SDt were tested using multivariate regression analyses using the statistical software R. However, the best fit was found when using a machine-learning regression method referred to as Partial Least Square (PLS) regression, which is a robust method in prediction and can handle data, which are strongly collinear and noisy (Johnson \& Wichern, 2007; Wang, et al., 2008). The resulting equation is provided below:

$$
\text { TETIndex }=\beta_{0}+\beta_{1} \mathrm{SDt}+\beta_{2}(\mathrm{SDv} / \mathrm{S})
$$

where, $\beta_{i}$ are the regression coefficients, and the other variables are as defined before. The unit of speed parameters is fps. The PLS regression result is in Table 4-6. The quality of the model shows an acceptable R-squared (the coefficient of determination) and Q-squared (the 
cross validated value which is calculated on the basis of the cross-validation). The error of the model based on the comparison of the estimated and observed TETIndex in the simulation is $14 \%$. Substituting the previously identified critical values of SDv, SDt and mean speed of $2 \mathrm{fps}, 8 \mathrm{fps}$ and $65 \mathrm{fps}$ respectively, in Equation 4-5, result in TETIndex of 0.03 , which is the critical value identified for this parameter earlier, further confirming that this model produce reasonable value.

Table 4-6: Coefficients and Quality of The TETIndex Estimation Based on PLS Regression

\begin{tabular}{|c|c|c|c|c|}
\hline Variable & Coefficient & SD & Lower bound (95\%) & Upper bound (95\%) \\
\hline$\beta_{0}$ & 0.010621 & 0.001 & 0.009 & 0.012 \\
\hline$\beta_{1}$ & 0.000241 & 0.0001 & 0.00007 & 0.001 \\
\hline$\beta_{2}$ & 0.637769 & 0.008 & 0.621 & 0.655 \\
\hline \multicolumn{5}{|c|}{ The Quality of the Model: } \\
\hline Statistic & Comp1 & Comp2 & & \\
\hline $\mathrm{Q}^{2}$ cum & 0.79 & 0.88 & & \\
\hline $\mathrm{R}^{2}$ cum & 0.79 & 0.88 & & \\
\hline \multicolumn{3}{|c|}{ MAPE $=14 \%$} & & \\
\hline
\end{tabular}

To validate the performance of the regression model presented in Equation 4-5, the model was tested for two locations with real-world data collected as part of the NGSIM program (I-80 and US101). This testing was conducted to assess the transferability of the regression model developed based on simulation data to other locations not used in the calibration of the simulation model. The model was tested under different sample size of data. The vehicles that were assumed to be equipped with technology that provides their trajectories for use in the model application were selected randomly from all vehicles in the traffic stream. Since the accuracy of the estimation is expected to depend on the random selection, a Monte Carlo analysis was used to account for the stochasticity by randomly selecting different vehicles from the traffic stream for each Monte Carlo run. Twenty Monte 
Carlo runs were conducted for each investigated market penetration of data, and the TETIndex was estimated based on speed parameters using Equation 4-5. Statistical tests indicated that 20 runs are sufficient. The results obtained with each market penetration were compared with the base value, which is the TETIndex calculated using Equation 3-5 based on the full NGSIM data. The MAPE and SDPE (Equations 4-2 and 4-3) used to assess the quality of the estimation.

The error of the model based on the MAPE and SDPE are reported in Table 4-7 for the two NGSIM datasets. As can be seen from Table 4-7, the developed regression model based on simulation was able to predict the TETIndex at an accuracy of $15 \%$ to $20 \%$ for locations that are different from the location used in the simulation.

Table 4-7: The Quality of Estimations of the Developed Regression Model for Different Sample based on NGSIM Data

\begin{tabular}{|c|c|c|c|c|c|}
\hline \multirow{2}{*}{\multicolumn{2}{|c|}{$\begin{array}{l}\text { Accuracy Measure } \\
\text { (Mean Value) }\end{array}$}} & \multicolumn{4}{|c|}{ Sample Size } \\
\hline & & \multirow{2}{*}{$\begin{array}{c}5 \% \\
20 \%\end{array}$} & \multirow{2}{*}{$\begin{array}{c}10 \% \\
19.8 \%\end{array}$} & \multirow{2}{*}{$\begin{array}{c}20 \% \\
19.6 \%\end{array}$} & \multirow{2}{*}{$\frac{100 \%}{19 \%}$} \\
\hline $\mathrm{I}-80$ & MAPE & & & & \\
\hline & SDPE & 0.78 & 0.51 & 0.45 & 0.29 \\
\hline \multirow[t]{2}{*}{ US101 } & MAPE & $16 \%$ & $15.5 \%$ & $15.8 \%$ & $15 \%$ \\
\hline & SDPE & 0.55 & 0.35 & 0.28 & 0.18 \\
\hline
\end{tabular}

\subsubsection{Real World CV Data Analysis}

As mentioned earlier, $\mathrm{CV}$ data was extracted to evaluate the performance of the application of the model for a freeway segment in Michigan. The data indicates that the speed on the segment varied between $45 \mathrm{fps}$ and $120 \mathrm{fps}$, and the peak period was between 3:00 P.M. and 5:00 P.M. The data was aggregated for each 5-minute interval. Then, the mean speed, average SDv, SDt and NO parameters were calculated based on vehicle trajectories for each interval. Since the TETIndex cannot be measured at low market penetrations of CV, 
Equation 4-5 was used to calculate the TETIndex based on speed parameters. Descriptive statistics of the measured traffic parameters for the segment were shown in Table 3-1.

Test location crash data for the year 2013 was also obtained and integrated with the traffic data for the time interval prior to crash occurrence. Crash was used as a binary dependent variable. A total of 35 crashes occurred in the test location in 2013. It is interesting that all crashes happened at TETindex values greater than 0.025 . More sever crashes happened at TETIndex higher than 0.03 and more property damage crashes happened at TETIndex higher than 0.05 , where the traffic conditions become congested.

Before applying statistical model to the crash, the Random Forest (RF) was used to rank the important variables on crash occurrences. RF is a non-parametric statistical method that is based on decision trees (Bishop, 2006). The R package "randomforest'(Liaw, 2002) was used to select the important variables. A higher accuracy represents a higher variable importance. The results are shown in Figure 4-9. As can be seen, the most important variables in predicting the occurrence of crashes are the TETIndex and NO.

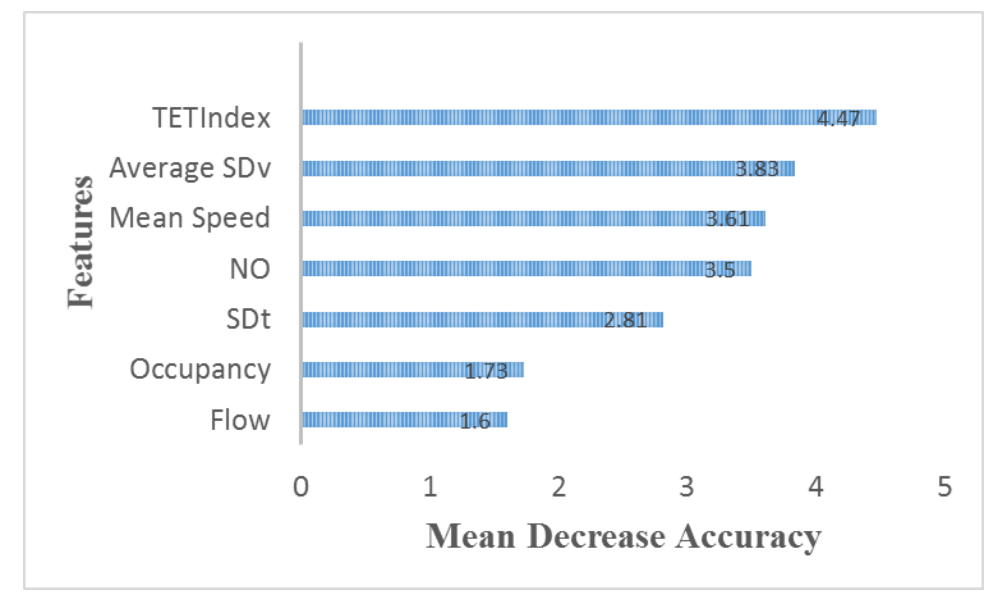

Figure 4-9: Variable Importance as Identified by the Random Forest 
Since some important variables identified by RF technique are correlated with each other, first, the Principal Component Analysis (PCA) was utilized to reduce the dimensionality of data and handle the collinearity between variables. The utilized number of components was selected based on the number of input features and projected variance (Krauss et al., 1997).

The Random Forest technique was used to fit a crash model to the data using the scikit-learn library in Python. The values of each input feature were standardized before use. As data samples are small, all data were used as a training dataset and $\mathrm{k}$-fold cross validation was used to estimate the accuracy of the model with 5-fold. Fine-tuning of model parameters was done using the Grid Search in the utilized tool, to tune the model by searching for the best hyper parameters and keeping the classifier with the highest accuracy. The overall accuracy with different utilized variables along with the tuned parameters are reported in Table 4-8. As can be seen, the developed model of the crash frequency for the Michigan test segment corresponds more with adding disturbance metrics to input variables than excluding them. This indicates that the utilized disturbances metrics are good indicators of traffic safety and they can use as inputs to predict crash in real-time operations.

Table 4-8: Summary of Tuned Parameters and Model Accuracy with Different Input Variables

\begin{tabular}{|l|l|c|c|}
\hline \multicolumn{2}{|l|}{ Evaluation } & All Variables & All Variables without Disturbance Metrics \\
\hline \multicolumn{2}{|l|}{ Overall Accuracy (SD) } & $0.82(+/-0.15)$ & $0.78(+/-0.21)$ \\
\hline \multirow{2}{*}{$\begin{array}{l}\text { Tuned } \\
\text { Parameters }\end{array}$} & max_depth & 3 & 3 \\
\cline { 2 - 4 } & max_features & 1 & 1 \\
\cline { 2 - 4 } & n_estimators & 100 & 100 \\
\hline
\end{tabular}

* $\mathrm{SD}=$ Standard Deviation

* n_estimators are the number of trees used in the ensemble, max_depth controls the depth of each tree and max_feature is the size of the random subsets of features to consider when splitting a node 


\subsection{Traffic State Identification}

This section describes the method developed for traffic state recognition (identification) from traffic operation point of view. Prior to conducting clustering for the purpose of traffic state recognition, the study performed an initial exploration of the three fundamental macroscopic variables (flow, density, and speed), for the simulated data as presented by the fundamental diagrams and shown in Figure 4-1. As mentioned in section 4.1, a Change-Point Regression with Gaussian Mixture Analysis was conducted using speed and occupancy data and the critical speed at capacity was found to be $65 \mathrm{fps}$. However, the fundamental diagram, shown in Figure 4-1 indicates that the relationships between the three fundamental macroscopic traffic features are very scattered and that the critical speed is somewhere between $50 \mathrm{fps}$ and $70 \mathrm{fps}$ and the critical density is between $40 \mathrm{vpml}$ to $60 \mathrm{vpml}$. This further indicates the need to use clustering and consider the microscopic features to identify breakdown.

As mentioned earlier, the GMM clustering was used to identify the uncertain traffic condition phase when the three macroscopic features (traffic flow, speed and density) were used in the clustering. Different numbers of components based on LOS (two to six) were used in the GMM clustering, and the SC was checked to determine the best number of components. It was found that the GMM with two components is the best investigated option. When examining the resulting GMM clusters, it was found that 365 datasets out of the 2997 are overlapping between two clusters and have probability of greater than zero to being on another cluster. Interestingly, these datasets are all in the mean speed range between $50 \mathrm{fps}$ to $70 \mathrm{fps}$. This confirms the stochasticity observed in the fundamental diagrams in Figure 4-1. 
The next step was to cluster the data in the uncertain traffic condition phase based on different combinations of macroscopic and microscopic features using the K-Means clustering algorithm. The results were then examined to determine the best combinations of features in separating the traffic state into two clusters of "breakdown" and "nonbreakdown". Please note that the goal of the study was defining traffic sate regarding breakdown, however, different number of clusters (k) was also attempted and the optimum number of clusters was found to be two.

The percentages of the data in the "breakdown" and "non-breakdown" clusters obtained using different combinations of features in the K-Means clustering and also those separated by the deterministic value of speed at capacity are shown in Table 4-9 based on whole dataset. As shown in Table 4-9, the percentage of the data in the breakdown cluster ranges from $32 \%$ to $42 \%$ depending on the utilized features.

The next step is to assess the results from the K-Means clustering presented in Table 4-9. First, the results were examined to determine the ability of different combinations to represent certain state correctly. Certain states were defined as speed lower than $50 \mathrm{fps}$ and greater than $70 \mathrm{fps}$ as the congested and uncongested conditions respectively. The percentage errors on certain conditions were then reported and are shown in Table 4-10. It can be seen that only five of the sixteen investigated combinations of the features produced zero errors in certain conditions. Note that additional combinations with flow and density, speed and flow and adding the SDt to the combinations were tried but were not reported in Table 4-9 and Table 4-10, since they did not produce improvements to the analysis.

Further examination was done by visually inspecting examples of trajectory data to determine the ability of the above options to isolate stop-and-go conditions from other 
conditions. Two random examples were selected to illustrate this visual inspection (Figure 4-10). Examples (a) and (b) have speed less than $65 \mathrm{fps}$ and should be categorized as breakdown according to the change-point regression based on the speed-occupancy relationship. However, Example (a) is stable while Example (b) is unstable (have stop-andgo conditions).

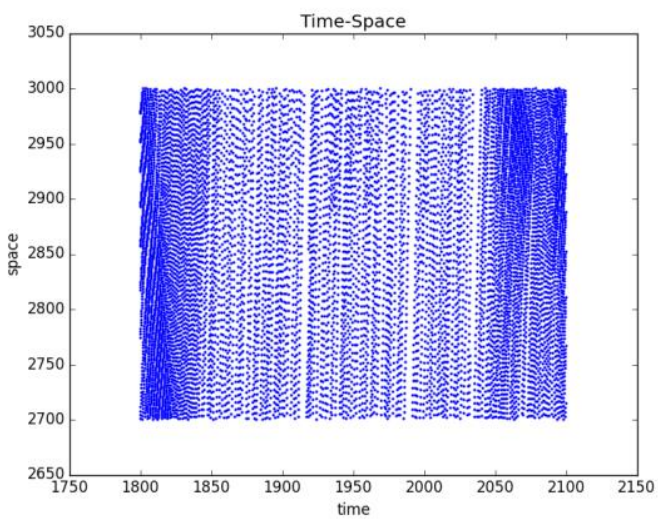

(a) Mean Speed $=56.6 \mathrm{fps}$ TETIndex $=0.03$ $\mathrm{NO}=9 \%$

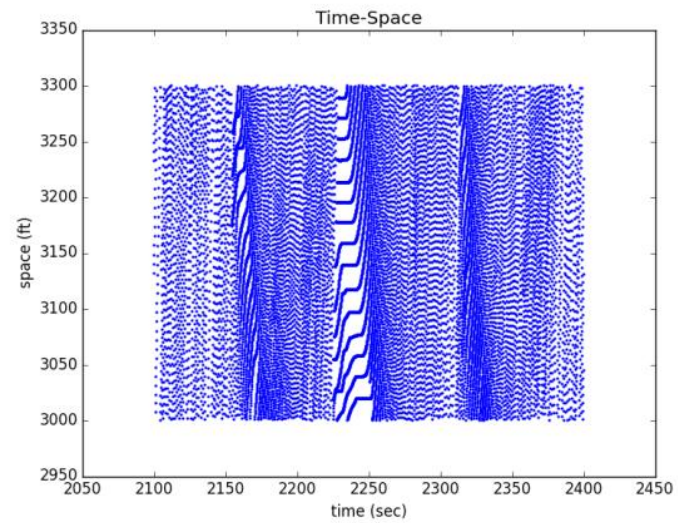

(b) Mean Speed=51.8 fps TETIndex $=0.05$ $\mathrm{NO}=20 \%$

Figure 4-10: Time-Space for the Two Examples of the Visual Inspection

Based on the results presented above regarding the error in assigning data to the congested /uncongested conditions in relation to speed, visual inspection, and the average SC; as shown in Table 4-10, it was determined that clustering with the "TETIndex, NO, Mean Speed and Occupancy" features, is the best clustering option among the investigated ones. The performance of utilizing the clustering based on these features (TETIndex, NO, Mean Speed and Occupancy), referred to as the "selected clustering" in the rest of this document, to identify "breakdown" and "non- breakdown" conditions was further compared to the utilization of clustering based on speed and occupancy only and also to using the 65 fps deterministic threshold determined according to the change-point regression. The results 
Table 4-9: Percentage of Traffic States in the Breakdown Cluster (Group1) and Non-Breakdown Cluster (Group2) when Using Different Combinations of Features

\begin{tabular}{|c|c|c|c|c|c|c|c|c|c|c|c|c|c|c|c|c|c|}
\hline \multirow[t]{2}{*}{ Clusters } & & \multicolumn{16}{|c|}{ Features Utilized in K-Means Clustering } \\
\hline & $\begin{array}{l}\text { S< } \\
65 \\
\text { fps }\end{array}$ & $\begin{array}{l}\text { TET } \\
- \\
\text { S- } \\
\text { Oc- } \\
\text { SDv }\end{array}$ & $\begin{array}{l}\text { NO- } \\
\text { S- } \\
\text { Oc- } \\
\text { SDv }\end{array}$ & $\begin{array}{l}\text { S- } \\
\text { Oc- } \\
\text { SDv }\end{array}$ & $\begin{array}{l}\text { TET } \\
-S- \\
\text { Oc }\end{array}$ & $\begin{array}{l}\text { TET } \\
-S- \\
\text { Oc- } \\
\text { NO }\end{array}$ & $\begin{array}{l}\text { S- } \\
\text { Oc- } \\
\text { NO }\end{array}$ & $\begin{array}{l}\text { S- } \\
\text { Oc }\end{array}$ & TET & SDv & $\mathrm{NO}$ & $\begin{array}{l}\text { S- } \\
\text { TET- } \\
\text { Flow }\end{array}$ & $\begin{array}{l}\text { S- } \\
\text { SDv- } \\
\text { Flow }\end{array}$ & $\begin{array}{l}\text { S- } \\
\text { NO- } \\
\text { Flow }\end{array}$ & $\begin{array}{l}\text { D- } \\
\text { Flow } \\
\text {-SDv }\end{array}$ & $\begin{array}{l}\text { D- } \\
\text { Flow } \\
-\mathrm{NO}\end{array}$ & $\begin{array}{l}\text { D- } \\
\text { Flow } \\
\text {-TET }\end{array}$ \\
\hline Group $1(\%)$ & 35.2 & 35.8 & 36.1 & 37.4 & 31.8 & 32.0 & 33.8 & 34.1 & 23.0 & 42.0 & 25.9 & 36.2 & 41.7 & 37.3 & 41.0 & 36.3 & 33.9 \\
\hline Group $2(\%)$ & 64.7 & 64.2 & 63.8 & 62.5 & 68.1 & 68.0 & 66.1 & 65.8 & 74.0 & 58.0 & 74.1 & 63.7 & 58.3 & 62.7 & 59.0 & 63.7 & 66.1 \\
\hline
\end{tabular}

*S=Space Mean Speed, Oc=Occupancy, $\mathrm{D}=$ Density, $\mathrm{SDv}=$ Average of standard deviations of individual vehicle's speeds, NO=Number of oscillations, TET=TETIndex 
Table 4-10: Evaluations of the Derived Clusters

\begin{tabular}{|c|c|c|c|c|c|c|c|c|c|c|c|c|c|c|c|c|c|}
\hline & \multicolumn{17}{|c|}{ Features Utilized in K-Means Clustering } \\
\hline & $\begin{array}{l}\mathrm{S}< \\
65 \\
\mathrm{fps}\end{array}$ & $\begin{array}{l}\text { TET- } \\
\text { S- } \\
\text { Oc- } \\
\text { SDv }\end{array}$ & $\begin{array}{l}\text { NO- } \\
\text { S- } \\
\text { Oc- } \\
\text { SDv }\end{array}$ & $\begin{array}{l}\text { S- } \\
\text { Oc- } \\
\text { SDv }\end{array}$ & $\begin{array}{l}\text { TET } \\
-\mathrm{S}- \\
\text { Oc }\end{array}$ & $\begin{array}{l}\text { TET } \\
-\mathrm{S}- \\
\text { Oc- } \\
\text { NO }\end{array}$ & $\begin{array}{l}\text { S- } \\
\text { Oc- } \\
\text { NO }\end{array}$ & $\begin{array}{l}\text { S- } \\
\text { Oc }\end{array}$ & TET & SDv & NO & $\begin{array}{l}\text { S- } \\
\text { TET- } \\
\text { Flow }\end{array}$ & $\begin{array}{l}\text { S- } \\
\text { SDv- } \\
\text { Flow }\end{array}$ & $\begin{array}{l}\text { S-NO- } \\
\text { Flow }\end{array}$ & $\begin{array}{l}\text { D- } \\
\text { Flow } \\
\text {-SDv }\end{array}$ & $\begin{array}{l}\text { D- } \\
\text { Flow } \\
\text {-NO }\end{array}$ & $\begin{array}{l}\text { D- } \\
\text { Flow- } \\
\text { TET }\end{array}$ \\
\hline $\begin{array}{l}\text { Percent Error } \\
\text { in Certain } \\
\text { Congested }\end{array}$ & $\mathbf{0}$ & $\mathbf{0}$ & $\mathbf{0}$ & $\mathbf{0}$ & 0.1 & $\mathbf{0}$ & 1.2 & $\mathbf{0}$ & 10.6 & $\mathbf{0}$ & 12.2 & 0.1 & $\mathbf{0}$ & 0.1 & 0.4 & 0.1 & 0.6 \\
\hline $\begin{array}{l}\text { Percent Error } \\
\text { in Certain } \\
\text { Uncongested }\end{array}$ & $\mathbf{0}$ & 0.1 & 0.1 & 0.48 & $\mathbf{0}$ & $\mathbf{0}$ & $\mathbf{0}$ & $\mathbf{0}$ & $\mathbf{0}$ & 7.3 & $\mathbf{0}$ & 0.8 & 6.29 & 1.29 & 5.9 & 1.7 & 0.64 \\
\hline \multicolumn{18}{|c|}{ Breakdown evaluation based on the three examples (corresponding with Figure 2) } \\
\hline Example (a) & $\mathrm{N}$ & $\mathrm{N}$ & $\mathrm{N}$ & $\mathrm{N}$ & $\mathbf{Y}$ & $\mathbf{Y}$ & $\mathbf{Y}$ & $\mathrm{N}$ & $\mathbf{Y}$ & $\mathrm{N}$ & $\mathbf{Y}$ & $\mathbf{Y}$ & $\mathrm{N}$ & $\mathrm{N}$ & $\mathrm{N}$ & $\mathbf{Y}$ & $\mathbf{Y}$ \\
\hline Example (b) & $\mathbf{N}$ & $\mathbf{N}$ & $\mathbf{N}$ & $\mathbf{N}$ & $\mathbf{N}$ & $\mathbf{N}$ & $\mathbf{N}$ & $\mathbf{N}$ & $Y$ & $\mathbf{N}$ & $Y$ & $Y$ & $\mathbf{N}$ & $\mathbf{N}$ & $\mathrm{Y}$ & $\mathbf{N}$ & $\mathbf{N}$ \\
\hline $\begin{array}{l}\text { Average } \\
\text { Silhouette }\end{array}$ & - & 0.67 & 0.7 & 0.69 & 0.7 & 0.69 & 0.68 & 0.71 & 0.73 & 0.77 & 0.76 & 0.53 & 0.56 & 0.54 & 0.53 & 0.52 & 0.51 \\
\hline
\end{tabular}

$* * \mathrm{~S}=$ Space Mean Speed, Oc=Occupancy, D=Density, SDv=Average standard deviations of individual vehicle’s speeds, NO=Number of oscillations, TET=TETIndex

** Bold colors shows clustering options that passed the evaluation correctly

$* * * \mathrm{Y}=$ stable condition (non- breakdown) $\mathrm{N}=$ unstable condition (breakdown)

$* * * *$ Certain congested is defined condition of speed lower than $50 \mathrm{fps}$ and certain

uncongested is defined conditions of speed greater than $70 \mathrm{fps}$ 
are reported in Table 4-11 and show that the clustering based on the four macroscopic and microscopic features can better account for the number of disturbance and disturbance duration that reflect the slow and go operations compared to the other two options. As can be seen from Table 4-11, the clustering with the "TETIndex, NO, Mean Speed and Occupancy", captures more disturbances in the breakdown cluster compared to using the deterministic value of speed at capacity and the clustering based on speed and occupancy. This selected clustering also reports the highest percentage of "non-breakdown" conditions for the whole dataset. It also clusters $56.1 \%$ of the uncertain phase (the measurements with speed between $50 \mathrm{fps}$ and $70 \mathrm{fps}$ ) as breakdown. Using the deterministic value of speed suggests $79.2 \%$ of the uncertain phase is in breakdown. It can be seen that the total improvement of the selected clustering compared to the clustering with the deterministic speed at capacity and with clustering with speed and occupancy are about $30 \%$ and $20 \%$, respectively. This means that $30 \%$ of the data in the uncertain phase considered as congested condition when using the deterministic value of a speed of $65 \mathrm{fps}$ are actually stable.

Table 4-11: Comparison of the Selected Clustering with a Deterministic Value of Speed and with Clustering based on Speed-Occupancy in Capturing the Amount of Disturbances in the Uncertain Phase (50 fps $<$ Mean Speed $<70 \mathrm{fps}$ )

\begin{tabular}{|l|c|c|c|}
\hline $\begin{array}{l}\text { Condition in the Uncertain Phase } \\
\text { (385 dataset out of 2997) }\end{array}$ & $\begin{array}{l}\text { Mean Speed } \\
65 \mathrm{fps}\end{array}$ & $\begin{array}{l}\text { Mean } \\
\text { Speed and } \\
\text { Occupancy }\end{array}$ & $\begin{array}{l}\text { TET, NO, } \\
\text { Mean } \\
\text { Speed, and } \\
\text { Occupancy }\end{array}$ \\
\hline Breakdown Condition (\%) & 79.2 & 70.6 & 56.1 \\
\hline \% of Reading NO $>20 \%$ in cluster of breakdown & 71.8 & 69.1 & 82.8 \\
\hline \% of Reading TET $>0.05$ in cluster of breakdown & 33.4 & 32.35 & 44.9 \\
\hline
\end{tabular}

Moreover, it was found that in the breakdown cluster, the value of TETIndex is greater than 0.05 and the NO is greater than $20 \%$. These two microscopic features could be considered together to decide if traffic flow perturbation could grow leading to 
breakdown or not. As described earlier. Table 4-12 shows the percentage of the captured disturbances from the selected clustering. As can be seen, the TETIndex of higher than 0.05 and NO higher than $20 \%$ can capture disturbances fully in breakdown conditions, while using the TETIndex and $\mathrm{NO}$ separately fails to identify the instability fully.

Table 4-12: The Results of Selected Clustering in Capturing Disturbances in Cluster of Breakdown

\begin{tabular}{|l|c|c|c|}
\hline Defined Traffic Conditions & $\begin{array}{l}\text { \% of Reading } \\
\text { TETIndex }>0.05\end{array}$ & $\begin{array}{l}\text { \% of Reading } \\
\text { NO> }>20 \%\end{array}$ & $\begin{array}{l}\text { \% of Reading } \\
\text { TETIndex }>0.05 \\
\text { And NO }>20 \%\end{array}$ \\
\hline Certain Congested & 91.7 & 95.2 & $100 \%$ \\
\hline $\begin{array}{l}\text { Breakdown } \\
\text { (Uncertain Phase) }\end{array}$ & 44.9 & 82.8 & $100 \%$ \\
\hline $\begin{array}{l}\text { Non-breakdown } \\
\text { (Uncertain Phase) }\end{array}$ & 7.05 & 38.2 & $0 \%$ \\
\hline Certain Uncongested & 0.1 & 0.85 & $0 \%$ \\
\hline
\end{tabular}

*From whole dataset $13 \%$ are in uncertain phase and $25 \%$ and $62 \%$ are in congested and uncongested conditions, respectively

The TETIndex of 0.05 and $\mathrm{NO}$ of $20 \%$ are also identified as values representing breakdown conditions based on the visualization from Figure 4-11. Figure 4-11 shows the relationship between the TETIndex and NO with the SDv, based on simulation results and NGSIM data (from US 101 in California). As can be seen, a TETIndex of 0.05 and NO of $20 \%$ are the change points where the diagrams bend and start a sharp increase further indicating that these values are good indicators of breakdown. Please note that although Figure 4-11 shows dataset from both VISSIM and NGSIM, only VISSIM data was used to develop the method and the NGSIM data was used to test the method. 

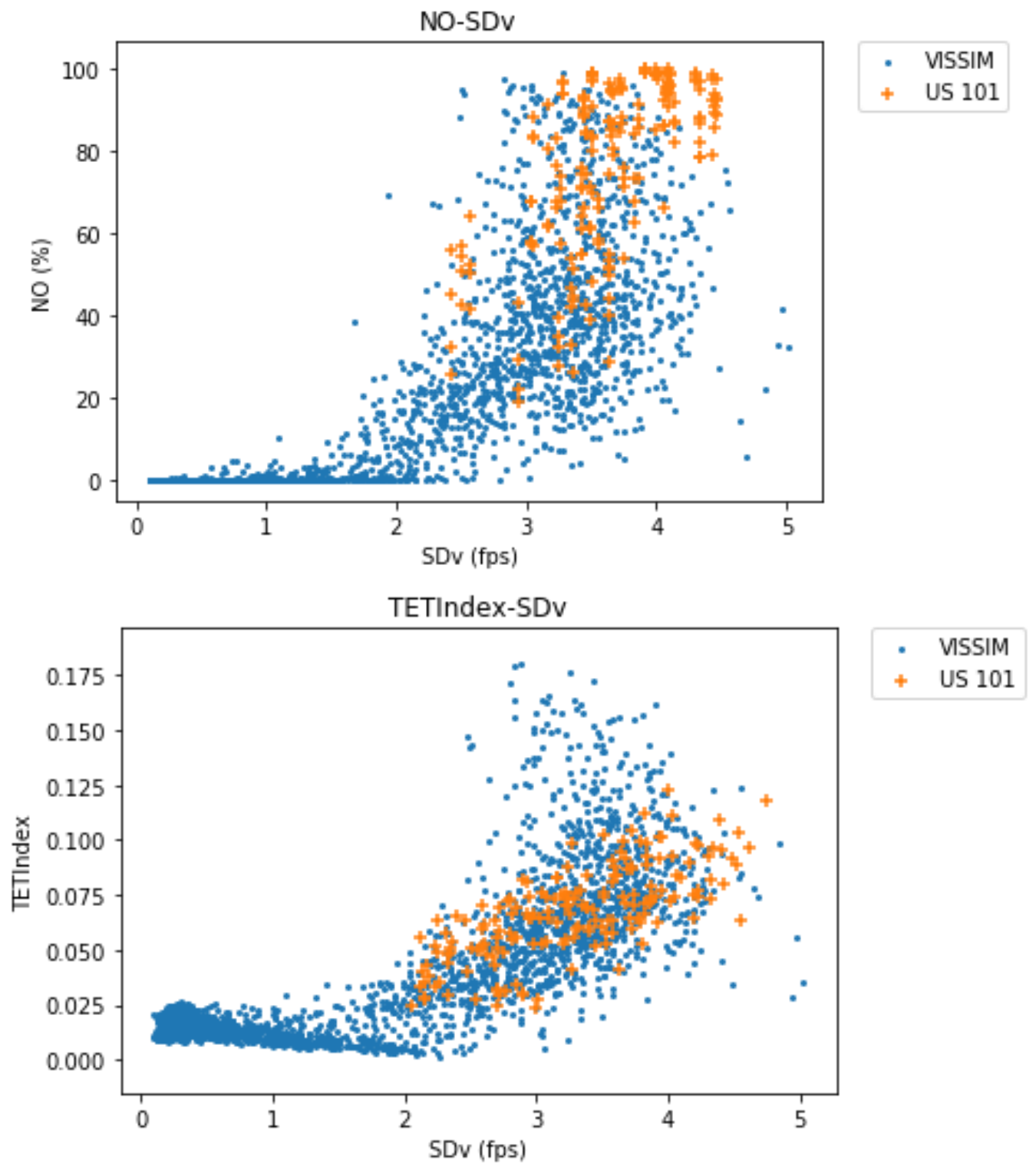

Figure 4-11: The Relationship between Disturbances with SDv

Testing the Method with Trajectory Data of NGSIM US 101:

The NGSIM dataset of US 101 in California was also used to test the proposed method. Although this dataset does not cover the different traffic states, the study investigated the inclusion of capturing the traffic disturbances in the traffic state identification. To be consistent with study by Lu et al. (2009) on NGSIM US 101 dataset 
about analyzing fundamental diagram, the same segment and same time window of one second was selected. The test segment is located in the upstream section of US101 in lane one which is about 550 feet long and for time period of 7:50 8:05 A.M. Lu et al( Lu et al., 2009) defined the critical density of around $95 \mathrm{vpml}$. As described in proposed method, first, the GMM clustering with macroscopic features was used and uncertain phase was identified. This uncertain phase located between density of $85 \mathrm{vpml}$ and $100 \mathrm{vpml}$. The identified uncertain traffic condition phase from the GMM analysis is then further analyzed using the K-Means clustering based on "TETIndex, NO, Mean Speed and Occupancy" to classify the uncertain phase into two clusters. The results are shown in Figure 4-12. The examination of Cluster 1 and Cluster 2 show that the TETIndex is between 0.06 to 0.099 in cluster 1 and is between 0.095 to 0.125 in Cluster 2. This indicates that this method not only can be used to identify breakdown from non-breakdown conditions, but also can be used to identify the level of congestion in the breakdown phase.

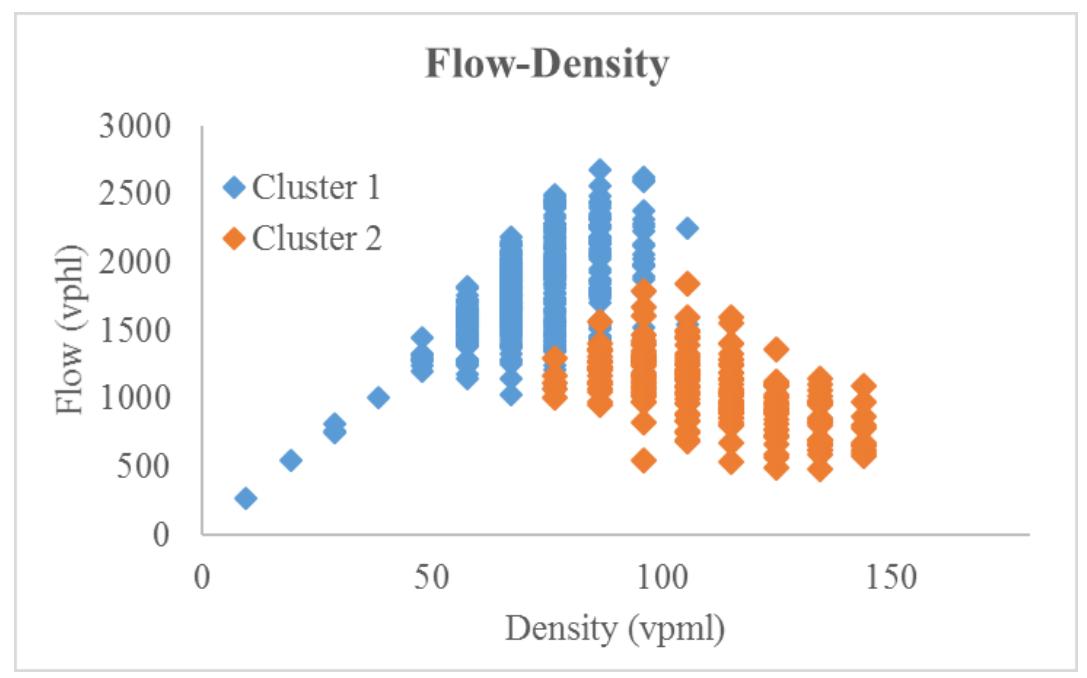

Figure 4-12: Data Clusters in the Test Segment of the NGSIM US101 Dataset 


\subsection{Traffic Flow Breakdown Prediction}

As stated earlier, the results of clustering discussed in the previous section, was used as a binary label to build the breakdown prediction model utilizing three machine learning methods that can act as classifiers of traffic patterns in real-time operations. Before applying a model to predict breakdown, the RF approach was used to rank the importance of the variables. RF is a non-parametric method that is based on decision trees (Liu et al., 2016). The R package "randomforest' was used to identify the importance of the variables. The RF was grown by building 200 decision trees and by randomly selecting two predictor variables at each split since this number of variables results in the minimum Out Of Bag (OOB) error. The assessment of the results was made using a metric referred to as Mean Decrease Accuracy. The results are shown in Figure 4-13. A higher accuracy value in the figure represents a higher variable importance. As can be seen in Figure 4-13 (a), the four most important variables in breakdown prediction are the mean speed, the TETIndex, NO, and occupancy. The RF was also applied to the real-world CV dataset. The results shown in Figure 4-13 (b) show that the four most important features to predict breakdown are the same four features identified for the simulation data. 


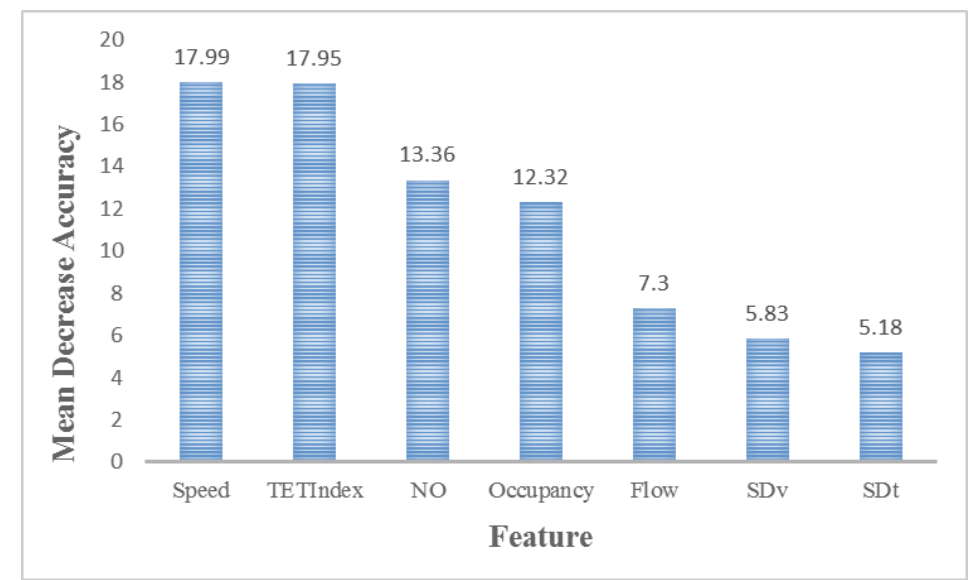

(a)

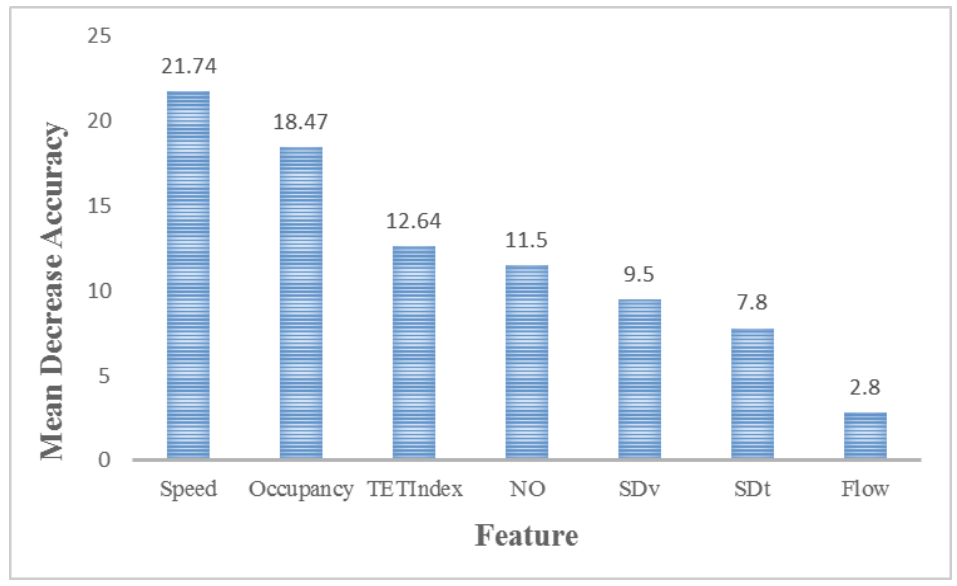

(b)

\section{Figure 4-13: Variable Importance Ranking Using Random Forest Technique (a) The Simulation Dataset (b) The Real-World CV Dataset}

The next step is to use the three selected machine learning methods (the SVM, RF, and $\mathrm{XGB}$ ) to develop models for real-time applications. The simulated data were split to training and testing datasets in 8:2 ratio with a random selection algorithm to make sure each dataset represent the maximum variance of data and to minimize having a biased dataset. For the real-world CV data, considering that the sample size is small, all data were used in the training and the k-fold cross validation was used to estimate the accuracy of the model with 5-folds. 
The features were temporally lagged for two 5-minute time intervals to allow the prediction of the traffic state in the following 5-minute interval. This means that the feature values estimated for the past two five minutes are used as inputs to the machine learning. The output is the predicted binary label of breakdown/non-breakdown for the next five minutes. To assess the effect of utilizing the defined disturbance and safety metrics in clustering and classification machine learning approaches on the accuracy of state prediction, two scenarios were studied as follows.

Scenario (A) involved the use of macroscopic and microscopic features as inputs to the machine learning. The utilized metrics are the flow, mean speed, occupancy, SDv, SDt, NO and TETIndex. In addition, the binary label obtained from clustering using the microscopic and macroscopic features, as discussed earlier, was used as a label that was also used in the training.

Scenario (B) involved all features in Scenario (A) without the two disturbance and safety metrics. Thus, the utilized metrics are the flow, mean speed, occupancy, SDv, and SDt. In addition, instead of using the binary label resulting from clustering, the binary label was assigned using the critical speed at capacity threshold for use as in the training.

Fine-tuning of the model parameters was done for each scenario and each method using the Grid Search, to tune the models by searching for the best hyper parameters and keeping the classifier with the highest accuracy. The final selected model parameters for each machine learning approach and each of the two scenarios (A and B) are reported in Table 4-13. In Table 4-13, the parameter $\mathrm{C}$ is the cost parameter of the error and shows the strength of the regularization. Gamma is a parameter for non-linear SVM, n_estimators is the number of trees used in each of the two ensembles (RF and XGB), learning _rate 
controls is used in fixing the error from the previous iteration, max_depth controls the depth of each tree and max_feature is the size of the random subsets of features to consider when splitting a node.

Table 4-13: Summary of Tuned Parameters with Three Scenarios for Each Classifier with Two Dataset

\begin{tabular}{|l|c|c|c|c|c|c|c|c|}
\hline Model & Scenarios & C & gamma & kernel & $\begin{array}{c}\text { learning } \\
\text { rate }\end{array}$ & $\begin{array}{c}\text { max__ } \\
\text { depth }\end{array}$ & $\begin{array}{c}\text { max_ } \\
\text { features }\end{array}$ & n_estimators \\
\hline \multirow{2}{*}{ SVM } & A & 1 & NA & Linear & NA & NA & NA & NA \\
\cline { 2 - 10 } & B & 10 & 0.1 & RBF & NA & NA & NA & NA \\
\hline \multirow{2}{*}{ RF } & A & NA & NA & NA & NA & 7 & 3 & 50 \\
\cline { 2 - 10 } & B & NA & NA & NA & NA & 7 & 2 & 150 \\
\hline \multirow{2}{*}{ XGB } & A & NA & NA & NA & 0.1 & 5 & 1 & 200 \\
\cline { 2 - 10 } & B & NA & NA & NA & 0.01 & 5 & 1 & 150 \\
\hline \multirow{2}{*}{ SVM } & A & 0.1 & NA & Linear & NA & NA & NA & NA \\
\cline { 2 - 10 } & B & 0.1 & NA & Linear & NA & NA & NA & NA \\
\hline \multirow{2}{*}{ RF } & A & NA & NA & NA & NA & 3 & 2 & 20 \\
\cline { 2 - 10 } & B & NA & NA & NA & NA & 3 & 1 & 20 \\
\hline \multirow{2}{*}{ XGB } & A & NA & NA & NA & 0.01 & 3 & 2 & 200 \\
\cline { 2 - 9 } & B & NA & NA & NA & 0.5 & 5 & 1 & 150 \\
\hline
\end{tabular}

* RBF= Radial Basis Function kernel SVM

The performance of each of the classifiers in the prediction in each scenario were assessed in terms of the overall accuracy, recall, precision, balanced accuracy, F1 Score, and confusion matrix, as reported in Table 4-14. In the confusion matrix, the rows are the predicted state and the columns are the actual state. The higher are the numbers on the diagonal of the matrix, the more accurate is the estimation. The other goodness of the models used in this study are defined below (Bishop, 2006).

The accuracy score is the number of the correct predictions made by the model. It indicates the overall performance of the model showing the fraction of the correct predictions $(\hat{\mathrm{y}})$ over $\mathrm{n}_{\text {samples }}$ and is computed as:

$$
\operatorname{Accuracy}(\mathrm{y}, \hat{\mathrm{y}})=\frac{1}{\mathrm{n}_{\text {samples }}} \sum_{\mathrm{i}=0}^{\mathrm{n}_{\text {samples }}{ }^{-1}} 1\left(\hat{\mathrm{y}}_{\mathrm{i}}=\mathrm{y}_{\mathrm{i}}\right)
$$


Precision, computed as below, is a measure of how accurate the positive predictions are. A high precision index indicates that most of the exampled labeled as positive are actually positive.

$$
\text { Precision }=\frac{\mathrm{TP}}{\mathrm{TP}+\mathrm{FP}}
$$

Recall refers to the coverage of the actual positive sample. In other words, a high recall index indicates that a class is correctly recognized.

$$
\text { Recall }=\frac{\mathrm{TP}}{\mathrm{TP}+\mathrm{FN}}
$$

In the above equations, TP are the true positives, TN are the true negatives, $\mathrm{FP}$ are the false positives (Type I error), and FN are the false negatives (Type II error).

The F1 score is a hybrid metric useful for unbalanced classes. The F1 score is computed as the harmonic mean of the precision and recall indices. It complements the precision index and is especially useful when uneven class distribution is present.

$$
\text { F1 score }=2 \times \frac{\text { Precision } \times \text { Recall }}{\text { Precision }+ \text { Recall }}
$$

When comparing the results of the evaluations in Table 4-14, it can be seen that Scenario (A) produced better results than Scenario (B) when used with all machine learning methods, confirming that the state estimation based on disturbance metrics combined with macroscopic metrics produce better results. All three investigated machine learning methods produced good accuracy with the RF approach producing somewhat better results than the other two methods.

The above results show the accuracy metrics for the overall prediction accuracy. However, this study also assessed the accuracy for a particular condition that is of specific importance to traffic management. This measure assesses the accuracy of the prediction of 
the occurrence of breakdown in the next five minutes when the states of the two previous five minutes are non-breakdown. This is very important to allow the activation of new management plans to address the breakdown before it occurs. Table 4-14 shows the accuracy of this prediction as the "\% Error in Predicting Transition to Congestion." Again, Scenario (A) produced better results with this metric compared to Scenario (B) for all investigated conditions. Also, the RF method appears from the results in Table 4-14 to produce somewhat better results than the other methods.

Table 4-14: Performance of the Three Classifiers in State Prediction based on Simulation Data

\begin{tabular}{|c|c|c|c|c|c|c|c|c|}
\hline Model & Scenarios & $\begin{array}{l}\text { Overall } \\
\text { Accuracy }\end{array}$ & Precision & Recall & $\begin{array}{l}\text { Balanced } \\
\text { Accuracy }\end{array}$ & F1 & $\begin{array}{l}\text { Confusion } \\
\text { Matrix }\end{array}$ & $\begin{array}{l}\text { \% Error in } \\
\text { Predicting } \\
\text { Transition to } \\
\text { Congestion }\end{array}$ \\
\hline \multirow[t]{2}{*}{ SVM } & A & 0.939 & 0.939 & 0.939 & 0.922 & 0.939 & $\begin{array}{l}{\left[\begin{array}{rr}130 & 4\end{array}\right]} \\
{\left[\begin{array}{rr}8 & 56\end{array}\right]}\end{array}$ & 7 \\
\hline & B & 0.924 & 0.924 & 0.924 & 0.906 & 0.924 & $\begin{array}{l}{\left[\begin{array}{lr}126 & 5\end{array}\right]} \\
{\left[\begin{array}{ll}10 & 57\end{array}\right]}\end{array}$ & 14 \\
\hline \multirow[t]{2}{*}{$\mathrm{RF}$} & A & 0.954 & 0.954 & 0.954 & 0.941 & 0.954 & $\begin{array}{l}{\left[\begin{array}{ll}131 & 3\end{array}\right]} \\
{\left[\begin{array}{ll}6 & 58\end{array}\right]}\end{array}$ & 5 \\
\hline & B & 0.934 & 0.935 & 0.934 & 0.913 & 0.940 & $\begin{array}{l}{\left[\begin{array}{lr}128 & 3\end{array}\right]} \\
{\left[\begin{array}{ll}10 & 57\end{array}\right]}\end{array}$ & 9 \\
\hline \multirow[t]{2}{*}{ XGB } & A & 0.949 & 0.950 & 0.949 & 0.930 & 0.949 & $\begin{array}{l}{\left[\begin{array}{rr}132 & 2\end{array}\right]} \\
{\left[\begin{array}{rr}8 & 56\end{array}\right]} \\
\end{array}$ & 7 \\
\hline & B & 0.924 & 0.924 & 0.924 & 0.901 & 0.924 & $\begin{array}{r}\left.\begin{array}{rr}125 & 3\end{array}\right] \\
{\left[\begin{array}{ll}12 & 58\end{array}\right]} \\
\end{array}$ & 19 \\
\hline
\end{tabular}

$*$ Note that the whole simulated dataset was 2968 data points where $20 \%$ was taken as test set with random selection. As the study considered 3 sequences of 3 time-interval of each 5 minutes, so the predicted size is about 198.

The method and scenarios tested using simulation trajectories as described earlier were also tested using $\mathrm{CV}$-data from a low market penetration deployment. As mentioned earlier, the evaluation in this study also utilizes $\mathrm{CV}$ data that was extracted to evaluate the performance of the application of the model for a freeway segment. The data indicates that the speed on the segment varied between $45 \mathrm{fps}$ and $120 \mathrm{fps}$, and the peak period was between 3:00 P.M. and 5:00 P.M. The results from applying the three machine learning 
techniques with Scenario (A) and Scenario (B) were evaluated using the 5-Fold cross validation. Table 4-15 shows the results from the evaluation. As with the simulated data, Table 4-15 shows that Scenario (A) had higher accuracy compared to Scenario (B) indicating the benefit of using the microscopic metrics. However, the machine learning algorithm that performed the best in this case was the XGB model.

Table 4-15: Performance of the Three Classifiers in State Prediction based on RealWorld CV Data

\begin{tabular}{|l|l|c|}
\hline Model & Scenarios & Overall Accuracy (SD) \\
\hline \multirow{3}{*}{ SVM } & A & $0.94(+/-0.15)$ \\
\cline { 2 - 3 } & B & $0.91(+/-0.15)$ \\
\hline \multirow{2}{*}{ RF } & A & $0.91(+/-0.14)$ \\
\cline { 2 - 3 } & B & $0.90(+/-0.15)$ \\
\hline \multirow{2}{*}{ XGB } & A & $\mathbf{0 . 9 5}(+/-0.13)$ \\
\cline { 2 - 3 } & B & $0.90(+/-0.12)$ \\
\hline
\end{tabular}

*SD=Standard Deviation

\subsection{Summary}

This chapter presented an exploration of the relationship between macroscopic and microscopic traffic parameters that are candidate for the recognition and prediction of the traffic operation and safety states. It then described the applications of the developed methodology in this study to estimate platooning, safety state and traffic operation state identification and prediction. In summary, it was found that adding the disturbance measures in the data clustering when identifying the traffic states will result in better traffic state recognition and prediction of the traffic flow state by capturing the disturbances in the traffic stream. Also, the study showed that the investigated disturbance measures and associated models and thresholds are significantly related to crash frequencies. The accuracy of developed models was also tested at low market penetration of real-world CV data. 


\section{CHAPTER V \\ CONCLUSIONS}

This chapter summarizes the findings of this research and outlines the precincts for future research.

\subsection{Summary and Conclusions}

This study proposed a methodology for the estimation and prediction of new measures based on $\mathrm{CV}$ data for potential use in off-line planning and in real-time management of traffic operations and safety. These measures include platooning measures, traffic disturbance measures, and safety surrogate measures. The percentage of platooned vehicles and the distribution of the platoon size were estimated based on surrogate measures that can be assessed using $\mathrm{CV}$ data at relatively low market penetrations of connected vehicles. The utilized measures are SDv and SDt. Relationships between the surrogate measures and the platooning measures were identified and utilized based on available trajectories data for different market penetrations of $\mathrm{CV}$. The results show that the percentage of vehicles in the platoon can be accurately and reliably estimated at relatively low $\mathrm{CV}$ market penetrations. For the platoon size distribution estimation, a low market penetration of 5\% is adequate when using the data for planning purposes based on multiple days. However, a minimum of $20 \%$ market penetration of $\mathrm{CV}$ is needed to estimate the platoon distribution for individual day operations.

This study also defined disturbance metrics and examined utilizing them as traffic safety and stability indicators for potential use in real-time operations. These disturbances metrics are the number of oscillations (NO) and a measure of disturbance durations index in terms of the time exposed time-to-collisions (TETIndex). The TET parameter has been 
used in the past as a safety surrogate measure. This study introduced its use for the first time as an indicator of traffic breakdown analysis and traffic safety analysis. Since TETIndex estimation cannot be measured at low market penetration of CV data, a regression model was derived based on speed parameters to estimate this parameter. Statistical testing of the model and associated parameters indicate that the model is significant and has a mean absolute percent error of $14 \%$. Then, the developed regression model was further validated using real-world trajectory data collected by the NGSIM program from two locations that were not used in the calibration of the simulation model. The results showed that the TETIndex can be estimated with low samples of trajectory data (e.g., data from low market penetration of trajectory connected vehicles) based on speed parameters with an error of around 15\%-20\%.

The application of the model to estimate safety risk utilizing trajectory data from a real-world deployment with low market penetration of $\mathrm{CV}$ data showed that including the utilized disturbance metrics allow better recognition of the crash risk. The study also confirmed that a TETIndex of 0.03 with NO of $10 \%$ can be used as thresholds above which the probability of perturbation growth and crash occurrence increase. This study found that that the investigated disturbance metrics can be use as indicators of unsafe conditions as part of decision support tools that include the activation of transportation management strategies to reduce the probability of unsafe traffic and ease traffic disturbances that have adverse impacts on traffic safety.

This study also utilized the defined microscopic disturbance and surrogate safety measures and examined the benefit of utilizing them in traffic state classification and prediction in combination with macroscopic traffic parameters. The combined macroscopic 
and microscopic measures are the TETIndex, NO, SDv, SDt, mean speed, traffic flow rate, and occupancy. The measures were used as inputs to a hybrid unsupervised clustering and three different supervised classifiers (SVM, RF, and XGB). The results indicate that the utilization of macroscopic measures by themselves in the traffic state estimation creates uncertainty with regard to traffic performance based on microscopic characteristics. This uncertainty covers in a relatively wide range of speed around the transition from the uncongested to the congested traffic conditions. The results of the evaluation performed in this study indicate that the combination of features that produced the best categorization of traffic state using clustering are the NO, TETIndex, average speed, and occupancy. The clustering results were compared to those obtained using a deterministic value of speed at capacity, derived using change-point regression and the results from clustering based only on speed and occupancy. It was also found that a TETIndex greater than 0.05 And NO greater than $20 \%$ can be used as criteria in the breakdown identification. The method was tested utilizing real-world NGSIM dataset. It was concluded that the proposed method of using the traffic disturbance parameters can also be used to categorize different levels of congestion.

The utilization of TETIndex and NO as disturbance metrics in combination with other metrics also increases the accuracy of traffic state prediction based on the results from the application of three supervised machine learning classifiers from both simulated trajectory dataset and real-world CV dataset. All three machine learning approaches investigated in this study (the SVM and two tree ensembles) performed well with slight variations in performance, depending on the specific case study data used in the investigation. It can be concluded that the investigated disturbance and surrogate metrics 
can be used as inputs to machine learning to predict traffic flow breakdown in terms of mobility and safety in real-time traffic operations. Such use is recommended as part of decision support tools that recommend the activation of transportation management strategies to reduce the probability of traffic breakdown and ease traffic disturbances.

\subsection{Research Contributions}

This research presented the shortcomings of current existing measurements and presented the estimation and prediction of new performance measures using trajectory data. The new performance measures were developed to estimate traffic safety and mobility by allowing the capture of disturbances by individual vehicles. The study reformed existing safety surrogate measures and defined disturbance metrics that reflect the stop-and-go or slow-and-go conditions by assessing the number of oscillations and TETIndex. TET has been widely used as a safety surrogate measure, but no one defined thresholds associated with this measure to activate transportation management strategies. For the first time, this research used the defined disturbance metrics as input variables to predict traffic safety and traffic flow breakdown. The Highway Capacity Manual (HCM) procedure for basic freeway segments categorizes the traffic states into six levels of service (LOS), and analysts have generally assumed that the breakdown occurs in the threshold between LOS E and F, where the demand exceeds capacity of the freeway segment. However, the breakdown can happen stochastically and not at a deterministic value of capacity. This study recommends adding the disturbance metrics in data clustering for better traffic state recognition and traffic flow breakdown prediction. 


\subsection{Limitation of the Research}

The outputs of this dissertation are limited to the data that are used in this study. To make any definite statement, researchers might need additional data from different places and different time periods. However, the developed methodologies are applicable to other locations to achieve the stated objectives.

\subsection{Recommendations for Future Research}

Future studies to extend this research could include the following:

- This research developed several performance measurements for measuring traffic safety and mobility on freeways using trajectory data. A future study will investigate performance measurements for signalized arterial streets.

- This study introduced new performance measurements to activate transportation management strategies. A future extension of this research can be the examination of the effectiveness of the management strategies on traffic operations from both the highway and vehicle sides. Transportation management strategies from the highway side can be ramp metering and variable speed limit to harmonize speed. To ease stop-and-go operations, the transportation management strategies from the vehicle (CV/CAV) side can include finding the optimum gap and speed of the vehicle to interact with the lead vehicle.

- In addition, in the coming years a proportion of the vehicles will be equipped with automated vehicle technologies. These technologies will impact the estimation of measures based on $\mathrm{CV}$ data. The described methodology in this research is also applicable to CAV. However, since the performance of automated vehicles will not 
reflect the performance of the rest of the traffic components, it is important to categorize traffic into vehicles without automation and vehicles with automation when calculating performance measures. 


\section{REFERENCES}

AASHTO. (2011). A Policy on Geometric Design of Highways and Streets, 6th Edition.

Abdel-Aty, M., \& Pande, A. (2005). Identifying Crash Propensity Using Specific Traffic Speed Conditions. Journal of Safety Research, 36(1), 97-108.

Al-Kaisy, A. \& Durbin,C. (2009). Platooning on Two-Lane Two-Way Highways: An Empirical Investigation. Journal of Advanced Transportation, 43, 71-88.

Arthur, D., \& Vassilvitskii, S. (2007). K-Means++: the Advantages of Careful Seeding. Proc ACM-SIAM Symposium on Discrete Algorithms., 8, 1027-1035.

Arvin, R., Kamrani, M., \& Khattak, A. J. (2019). How instantaneous Driving Behavior Contributes to Crashes at Intersections: Extracting Useful Information from Connected Vehicle Message Data. Accident Analysis and Prevention, 127, 118-133.

Balas, V. E., \& Balas, M. M. (2007). Driver Assisting by Inverse Time to Collision. 2006 World Automation Congress, WAC'06, 1-6.

Bishop, Ch. M. (2006). Pattern Recognition and Machine Learning. Springer.

Bullen, A. G. R. (1972). Strategies for Practical Expressway Control. Journal of the Transportation Engineering Division, 98(3), 599-605.

Chang, G. L., \& Xiang, H. (2003). The Relationship Between Congestion Levels and Accidents. State Highway Adiministration.

Chatterjee, S., Roy, D., Chakraborty, S., \& Roy, S. K. (2017). Level of Service Criteria on Indian Multilane Highways based on Platoon Characteristics. 96st Annual Meeting of Transportation Research Board, Washington DC.

Chen, X., Li,Z. Li,L., \& Qixin. S. (2014). A Traffic Breakdown Model Based on Queuing Theory. Network and Spatial economics, Springer, 14, 485-504.

Dehman, A. (2014). Breakdown Maturity Phenomenon at Wisconsin Freeway Bottlenecks. Transportation Research Record: Journal of the Transportation Research Board, 2395(1), $1-11$.

Dijkstra, A., Marchesini, P., Bijleveld, F., Kars, V., Drolenga, H., \& Maarseveen, M. (2010). Do Calculated Conflicts in Microsimulation Model Predict Number of Crashes? Transportation Research Record: Journal of the Transportation Research Board, 2147(1), 105-112. 
Dong, J., \& Mahmassani, H. S. (2012). Stochastic Modeling of Traffic Flow Breakdown Phenomenon: Application to Predicting Travel Time Reliability. IEEE Transactions on Intelligent Transportation Systems, 13(4), 1803-1809.

Elefteriadou, L. (2017). Freeway-Merging Operations: A Probabilistic Approach. IFAC Proceedings Volumes, 30(8), 1283-1288.

Elefteriadou, L., Roger, P. R., \& Mcshane, W. R. (1995). Probabilistic Nature of Breakdown at Freeway Merge Junctions. Transportation Research Record: Journal of the Transportation Research Board, 1484, 80-89.

Elfar, A., Talebpour, A., \& Mahmassani, H. S. (2018). Machine Learning Approach to Short-Term Traffic Congestion Prediction in a Connected Environment. Transportation Research Record: Journal of the Transportation Research Board, 2672(45), 185-195.

Fan, R., Wang, W., Liu, P., \& Yu, H. (2013). Using VISSIM Simulation Model and Surrogate Safety Assessment Model for Estimating Field Measured Traffic Conflicts at Freeway Merge Areas. IET Intelligent Transport Systems, 7(1), 68-77.

FHWA. (2007). US Highway 101 Dataset.

Gattis, J., Alguire,M., Townsend, K. \& Rao, S. (1997). Rural Two-Lane Passing Headways and Platooning. Transportation Research Record: Journal of the Transportation Research Board,, 1579, 27-34.

Gettman,D.,\& Head,L.(2003).Surrogate Safety Measures from Traffic Simulaton Models.FHWA.

Guido, G., Vitale, A., Astarita, V., Saccomanno, F., Giofré, V. P., \& Gallelli, V. (2012). Estimation of Safety Performance Measures from Smartphone Sensors. Procedia - Social and Behavioral Sciences, 54, 1095-1103.

Hale, D. K., Button, L., Argote, J., Joshi, C., Zhang, X., Kondyli, A., \& Bared, J. G. (2019). Calibration of Microsimulation Car-Following Models for Narrow Freeway Lanes. 98st Annual Meeting of Transportation Research Board, Washington DC.

Han, Y., \& Ahn, S. (2017). Stochastic Modeling of Breakdown at Freeway Merge Bottleneck. 96th Annual Meeting Transportation Research Board, Washington DC.

Hartman,K., Goplakrishna,D. \& Kitchener, F. M. (2016). Performance Measurement and Evaluation Support Plan. ICF/Wyoming CV Pilot.

Hayward, J. (1972). Near Miss Determination Through Use of a Scale of Danger. Report TTSC-7115. 
Hale. (2018). Scale, Standardize, or Normalize with Scikit-Learn-Towards Data Science.

Henclewood, D., Abramovich, M.,\& Yelchuru, B. (2014). Safety Pilot Model Deploymentone Day Sample Data Environment Data Handbook. USDOT Research and Technology Innovation Administrations.

Herman, R., Montroll, E. W., Potts, R. B., \& Rothery, R. W. (1959). Traffic Dynamics: Analysis of Stability in Car Following. Operations Research, 7(1), 86-106.

HCM (2016). Highway Capacity Manual, Sixth Edition

ITS DataHub. https://www.its.dot.gov/data/

Jiang, R., Hu, M.B., Zhang, H.M., Gao, Z.Y., \& Jia,B. (2015). On Some Experimental Features of Car-Following Behavior and How to Model Them. Transportation Research Part B, 80, 338-354.

Jiang, R., Jin, C. J., Zhang, H. M., Huang, Y. X., Tian, J. F., Wang, W., \& Jia, B. (2018). Experimental and Empirical Investigations of Traffic Flow Instability. Transportation Research Part C: Emerging Technologies, 94, 83-98.

Jingxin, X., \& Mei, C. (2007). Defining Traffic Flow Phases Using Intelligent Transportation Systems-Generated Data. Journal of Intelligent Transportation Systems: Technology, Planning, and Operations, 11(1), 15-24.

Johnson, R. A., \& Wichern, D. W. (2007). Appllied Multivariate Statistics Analysis.

Kamrani, M., Arvin, R., \& Khattak, A. J. (2018). Extracting Useful Information from Basic Safety Message Data: An Empirical Study of Driving Volatility Measures and Crash Frequency at Intersections. Transportation Research Record: Journal of the Transportation Research Board, 2672(38), 290-301.

Khajeh Hosseini, M., \& Talebpour, A. (2019). Traffic Prediction using Time-Space Diagram: A Convolutional Neural Network Approach. Transportation Research Record: Journal of the Transportation Research Board , 2673(7),425-435

Kianfar, J., \& Edara, P. (2013). A Data Mining Approach to Creating Fundamental Traffic Flow Diagram. Procedia - Social and Behavioral Sciences, 104, 430-439.

Kidando, E., Moses, R., Kitali, A. E., Kwigizile, V., Lyimo, S. M., Chimba, D., \& Sando, T. (2018). Exploring the Influence of Rainfall on a Stochastic Evolution of Traffic Conditions. 97th Annual Meeting Transportation Research Board, Washington DC. 
Kidando, E., Moses, R., \& Sando, T. (2019). A Statistical Approach for Estimating Speed Threshold for Traffic Breakdown Event Identification: a Model Accounting for Data Variations. 98th Annual Meeting Transportation Research Board, Washington DC.

Kidando, E., Moses, R., Ghorbanzadeh, M., Ozguven, E.(2018). Traffic Operation and Safety Analysis on an Arterial Highway: Implications for Connected Vehicle Applications. International Conference on Intelligent Transportation Systems, 2753-2758.

Ko, J., \& Guensler, R. (2005). Characterization of Congestion Based on Speed Distribution: A Statistical Approach Using Gaussian Mixture Model. 84st Annual Meeting of Transportation Research Board, Washington DC.

Kondyli, A. (2009). Breakdown Probability Model at Freeway-Ramp Merges Based On Driver Behavior. University of Florida.

Kondyli, Alexandra, Elefteriadou, L., Brilon, W., Hall, F. L., Persaud, B., \& Washburn, S. (2013). Development and Evaluation of Methods for Constructing Breakdown Probability Models. Journal of Transportation Engineering, 139(9), 931-940.

Krauss, S. , Wanger,P. \&Gawron C. (1997). Metastable States in a Microscopic Model of Traffic Flow. Physical Review E, 55(5), 5597.

Kuang, Y., Qu, X., \& Wang, S. (2015). A Tree-Structured Crash Surrogate Measure for Freeways. Accident Analysis \& Prevention, 77, 137-148.

Kühne, R. (1984). Macroscopic Freeway Model for Dense Traffic Stop-Start Waves and Incident Detection. 9th International Symposium on Transportation and Traffic Theory, $21-42$.

Laflamme, E. M., \& Ossenbruggen, P. J. (2017). Effect of Time-of-Day and Day-of-theWeek on Congestion Duration and Breakdown: A Case Study at a Bottleneck in Salem, NH. Journal of Traffic and Transportation Engineering (English Edition), 4(1), 31-40.

Lay, M. (1986). Handbook of Road Technology. Gordon and Breach.

Lee, C., Saccomanno, F., \& Hellinga, B. (2002). Analysis of Crash Precursors on Instrumented Freeways. Transportation Research Record: Journal of the Transportation Research Board, 1784(1), 1-8.

Li, Y., Wang, H., Wang, W., Xing, L., Liu, S., \& Wei, X. (2017). Evaluation of the Impacts of Cooperative Adaptive Cruise Control on Reducing Rear-End Collision Risks on Freeways. Accident Analysis and Prevention, 98, 87-95.

Li, Z., Ahn, S., Chung, K., Ragland,D. R., Wang, W., \&Whon Yu, J. (2014). Surrogate Safety Measure for Evaluating Rear-End Collision Risk Related to Kinematic Waves Near 
Freeway Recurrent Bottlenecks. Accident Analysis and Prevention, 64, 52-61.

Liaw,A., \&Wiener,M. (2002). Classification and Regression by RandomForest. $R$ News, 2 , $18-22$.

Liu, S., McGree, J., Ge, Z., \& Xie, Y. (2016). Finding Groups in Data. Computational and Statistical Methods for Analysing Big Data with Applications, 29-55.

Lu, G., Cheng, B., Kuzumaki, S., \& Mei, B. (2011). Relationship between Road Traffic Accidents and Conflicts Recorded by Drive Recorders. Traffic Injury Prevention, 12(4), $320-326$.

Lu, X. Y., Varaiya, P., \& Horowitz, R. (2009). Fundamental Diagram modelling and analysis based NGSIM data. IFAC Proceedings Volumes (IFAC-PapersOnline), 42(15), $367-374$.

Mahmoudzadeh, A., Razi-Ardakani, H., Kermanshah, M.(2019). Studying Crash Avoidance Maneuvers Prior to an Impact Considering Different Types of Driver's Distractions.Transportation Research Procedia ,37, 203-210.

McShane. (2011). Traffic engineering. Pearson.

Minderhoud,M.M.,\&Bovy,P.H.(2001).Extended Time-to-Collision Measures for Road Traffic Safety.Accident Analysis and prevention,33, 89-97.

NCHRP, report 551. (2006). Performance Measures and Targets for Transportation Asset Management.

Neal, R. M. (2007). Pattern Recognition and Machine Learning. Technometrics, 49(3), 366-366.

Oh, C., Park, S., \& Ritchie, S. G. (2006). A method for Identifying Rear-End Collision Risks Using Inductive Loop Detectors. Accident Analysis and Prevention, 38(2), 295-301.

Ozbay, K., Yang, H., G., Bartin, B., \& Mudigonda, S. (2007). Derivation and Validation of a New Simulation-based Surrogate Safety Measure. 86st Annual Meeting of Transportation Research Board, Washington DC.

Peng, Y., Abdel-Aty, M., Shi, Q., \& Yu, R. (2017). Assessing the Impact of Reduced Visibility on Traffic Crash Risk Using Microscopic Data and Surrogate Safety Measures. Transportation Research Part C: Emerging Technologies, 74, 295-305.

Pueboobpaphan, R., \& Arem, B. (2011). Driver and Vehicle Characteristics and Platoon and Traffic Flow Stability. Transportation Research Record: Journal of the Transportation Research Board, 2189(1), 89-97. 
Rahman, A. and Lowenns,N. (2012). Analysis of Rainfall Impacts on Platooned Vehicle Spacing and Speed. Transportation Research Part F, 15, 395-403.

Rahman, M. S., \& Abdel-Aty, M. (2018). Longitudinal Safety Evaluation of Connected Vehicles' Platooning On Expressways. Accident Analysis and Prevention, 117, 381-391.

Rahmani, R., Sun, C., Edara, P., Brown, H., \& Zhu, Z. (2016). Freeway Work Zone Crash Prediction Models Using Missouri Data. 95th Annual Meeting of Transportation Research Board, Washington DC.

Rakha,H., and Gao,Y. (2010). Calibration of Steady-State Car-Following Models Using Macroscopic Loop Detector Data. VT-2008-01 DTRS 99-G-003, Virginia Department of Transportation and the U.S.Department of Transportation.

SAE International. (2009). J2735: Dedicated Short Range Communications (DSRC) Message Set Dictionary.

Shiomi, Y, Toshio,Y. \& Ryuichi, K. (2011). Platoon-Based Traffic Flow Model for Estimating Breakdown Probability at Single-Lane Expressway Bottlenecks. Transportation Research Part B, 45, 1314-1330.

Son, B, Taewan, K., Kim, H. J.\& Lee,S. (2004). Probabilistic Model of Traffic Breakdown with Random Propagation of Disturbance for ITS Application. Lecture Notes in Artificial Intelligence, 45-51.

Sun. D \& R. F. Benekohal. (2005). Analysis of Work Zone Gaps and Rear-End Collision Probability. Journal of Transportation and Statistics.

Swaroop, D., \& Rajagopal, K. R. (1999). Intelligent Cruise Control Systems and Traffic Flow Behavior. American Society of Mechanical Engineers, Dynamic Systems and Control Division, 67, 373-380.

Tian, J., Jiang, R., Jia, B., Gao, Z., \& Ma, S. (2016). Empirical Analysis and Simulation of The Evolution Concavity Of Traffic Oscillations.Transportation research Part B,93,338354.

Treiber, M., \& Kesting, A. (2013). Traffic Flow Dynamics: Data, Models and Simulation. Springer.

Van Lint, \&Van,C. (2012). Short-Term Traffic and Travel Time Prediction Models. Artificial Intelligence Applications to Critical Transportation Issues, 22, 22-41.

Vogel, K. (2002). What Characterizes A "Free Vehicle" In an Urban Area? Transportation Research Part F: Traffic Psychology and Behavior, 5, 15-29. 
Wang, H., Rudy, K. , Jia, L. \& Daiheng, N. (2010). Calculation of Traffic Breakdown Probability to Optimize Link Throughput. Applied Mathematical Modelling. 3376-3389.

Wang, W., Chen, S., \& Qu, G. (2008). Incident Detection Algorithm Based on Partial Least Squares Regression. Transportation Research Part C: Emerging Technologies, 16(1), 5470.

Wright, J., Kyle Garrett,J., Hill,J., Krueger,G., Evans,J., Andrews,S., Wilson,C.K., \& Rajbhandari,R. (2014). National Connected Vehicle Field Infrastructure Footprint Analysis. FHWA-JPO-14-125. United States. Department of Transportation. Intelligent Transportation Systems Joint Program Office.

Yang, X., J., Zheng, j.,\& Sun,J. (2015). Empirical Analysis of Platoon Characteristics: Evidence from Trajectory Data at Two Freeway On-Ramp Bottlenecks. CICTP, ASCE.

Yeon, J., Hernandez, S., \& Elefteriadou, L. (2009). Differences in Freeway Capacity by Day of the Week, Time of Day, and Segment Type. Journal of Transportation Engineering, 135(7), 416-426.

Zhang, Z., Ding, F., Zhou, Y., Ahn, S., \& Ran, B. (2019). Deep Long Short-Term Memory Network Based Long-Term Vehicle Trajectory Prediction. 98st Annual Meeting of Transportation Research Board, Washington DC.

Zheng, Z. (2012). Empirical Analysis on Relationship between Traffic Conditions and Crash Occurrences. Procedia - Social and Behavioral Sciences, 43, 302-312.

Zheng, Z., Ahn, S., \& Monsere, C. M. (2010). Impact of traffic oscillations on freeway crash occurrences. Accident Analysis and Prevention, 42(2), 626-636. 
VITA

LEILA AZIZI

\section{EDUCATION}

2005

2009

2019

2019

2018

2018

2018

2018

2018

2017

2017

2017-Present

$2016-2017$

$2017-2018$
B.S., Track and Railway Structures Engineering Iran University of Science and Technology, Tehran, Iran

M.S., Civil Engineering (Transportation Planning Engineering) Iran University of Science and Technology, Tehran, Iran

Ph.D. Civil Engineering (Transportation Engineering)

Florida International University, Miami, Florida, USA

Poster Presentation Competition, Third Place, STRIDE

Fang Zhao Women in Engineering Leadership, FIU

Helene M. Overly Memorial Scholarship, WTS South Florida

Student Government Association Scholarship, FIU

Poster Presentation Competition, Second Place, FIU

Chair Shadow, WTS International

Anne S. Brewer Scholarship, ITS Florida

Essay Competition, First Place, GCCITE, South Florida

Paper reviewer (ASCE, TRB/TRR)

Vice President, WTS Student Chapter at FIU

President, WTS Student Chapter at FIU

\section{PUBLICATIONS AND PRESENTATIONS}

Azizi, L., M. Hadi, T. Wang, and M. Kamrani, Estimation of Freeway Traffic Safety Utilizing Disturbance Metrics Based on Trajectory Data, Accepted for Presentation at the 99th Annual Meeting of Transportation Research Board, and considered for publication at the Transportation Research Record: Journal of the Transportation Research Board, 2020.

Azizi, L., M. Hadi, M. Aghili, and S. Mokhtari, Real-Time Prediction of Traffic State on Freeways Based on Trajectory Data: A Machine Learning Approach, Accepted for Presentation at the 99th Annual Meeting of Transportation Research Board, and considered for publication at the Transportation Research Record: Journal of the Transportation Research Board, 2020. 
Azizi, L., and M. Hadi, Freeway Traffic Flow State Identification with Consideration of Disturbances by Individual's Vehicle: A Hybrid Scheme of Gaussian Mixture Model and K-Means Clustering, Accepted for Presentation at the 99th Annual Meeting of Transportation Research Board, 2020.

Azizi, L. Freeway Performance Measurement in a Connected Vehicle Environment, Accepted for Workshop, Doctoral Student Research in Transportation Operations and Traffic Control, at the 99th Annual Meeting of Transportation Research Board, 2020.

Azizi, L., and M. Hadi, Estimation of Mobility Performance of Freeways Based on Time Exposed Time to Collision Using Trajectory Data in a Connected Environment. Oral Presentation, TRB Performance and Data in Transportation Decision Making Conference, 2019.

Azizi, L., and M. Hadi, Traffic Flow Stability Analysis and Performance Evaluation Methods Based on Connected Vehicle Data. International Conference on Transportation \&Development (ICTD), ASCE, 2019.

Hadi, M., L. Azizi, and T. Wang, Performance Measurement and Management Using Connected and Automated Vehicle Data. A Reported Produced for the STRIDE USDOT University Transportation Research Center, 2019.

Azizi, L. M.S. Iqbal, and M. Hadi, Estimation of Freeway Platooning Measures Using Surrogate Measures Based on Connected Vehicle Data, Proceedings of the 97th Annual Meeting of the Transportation Research Board, Washington DC, 2018.

Azizi, L. Advances in Modeling and Incorporating Big Data in Planning. WTS International conference, NY, 2017.

Hadi, M., Y. Xiao, T. Wang, S.F. Qom, L. Azizi, J. Jia, A. Massahi, and M.S. Iqbal, Framework for Multi-Resolution Analyses of Advanced Traffic Management Strategies, Final Report No. BDV29-977-19, Prepared for FDOT, 2016.

Azizi, L., M. Hadi and Y. Xiao, Comparison Real-time Travel Time Prediction Methods, 4th Annual UTC Conference for the Southeastern Region, Knoxville, 2016.

Azizi, L., M. Hadi and Y. Xiao, Exploring Advanced Features of the CUBE Analyst Drive Performance as Part of the Dynamic Origin-Destination Matrix Estimation Process, FUTURA'16 Citilabs International User Conference, 2016.

Azizi, L and A. Sheikholeslami, Safety Effect of U-turns Conversions in Tehran: Empirical Bayes Observational Before-and-After Study and Crash Prediction Models, Journal of Transportation Engineering, ASCE, Vol.139, pp.101-108, 2013. 